\title{
Anéis e Módulos Distributivos
}

Leonardo Linhares Rodrigues

\author{
DISSERTAÇÃO APRESENTADA \\ $\mathrm{AO}$ \\ INSTITUTO DE MATEMÁTICA E ESTATÍSTICA \\ DA \\ UNIVERSIDADE DE SÃO PAULO \\ PARA \\ OBTENÇÃO DO GRAU \\ $\mathrm{DE}$ \\ MESTRE EM MATEMÁTICA PURA
}

Área de Concentração: Álgebra

Orientador: Prof. Dr. Francisco César Polcino Milies

- São Paulo, Maio de 2004 - 


\title{
Anéis e Módulos Distributivos
}

\author{
Este exemplar corresponde à redação final \\ da dissertação de mestrado devidamente \\ corrigida e defendida por \\ Leonardo Linhares Rodrigues \\ e aprovada pela comissão julgadora.
}

São Paulo, maio de 2004.

Banca examinadora:

- Prof. Dr. Francisco César Polcino Milies (Orientador) - IME-USP

- Prof. Dr. Mikhajolo Dokuchaev - IME-USP

- Prof. Dr. Miguel Ferrero - UFRGS 


\section{Agradecimentos}

Dedico este trabalho às seguintes pessoas:

À minha querida esposa Ignês, pela paciência para aturar as intermináveis horas de "gagá" necessárias para a confecção deste trabalho.

A meus queridos familiares Ridj, Mota, Zeca e Luiz Afrânio, pelo amor e pela torcida.

A meus colegas e amigos Anliy, Márcio e Pavlos, pelo companheirismo, pela amizade e pela rica troca de idéias matemáticas e filosóficas.

Ao professor Luiz Antonio Peresi, pelas longas e esclarecedoras conversas e por despertar em mim o gosto pela Álgebra.

A meu orientador César Polcino Milies, pela oportunidade de me orientar. Foi uma grande satisfação poder contar com sua longa experiência.

"Was ist Glück? Das Gefühl, daß die Macht wächst, daß ein Widerstand überwunden wird". Friedrich Nietzsche 


\section{Sumário}

Abstract

Agradecimentos $\quad \mathrm{V}$

Introdução vii

Índice de Notação $\quad$ ix

1 Conceitos Básicos 1

1.1 Anéis e Módulos Simples . . . . . . . . . . . . . . . . . . . 1

1.2 Condições de Finitude . . . . . . . . . . . . . . . . . 2

1.3 Radical de Jacobson . . . . . . . . . . . . . . . . . . . . 4

1.4 Anéis Semisimples e Semiprimários ... . . . . . . . . . 5

1.5 Idempotentes . . . . . . . . . . . . . . . . . . 7

1.6 Módulos ..................... . . 8

2 Anéis Semiperfeitos e Perfeitos 11

2.1 Anéis Locais . . . . . . . . . . . . . . . . . . . . . . . . . . . . . . . . . . . . . . . . . 14

2.2 Anéis Semiperfeitos . . . . . . . . . . . . . . . . . . . . . . . . . . . . . . . . . . 14

2.3 Anéis Perfeitos . . . . . . . . . . . . . . . . . . . . . . . . . . . . . . . . . . . . 38

2.4 Caracterizações Homológicas . . . . . . . . . . . . . . . . . . . 33

2.5 Exemplos . . . . . . . . . . . . . . . . 46

3 Anéis e Módulos Distributivos $\quad 51$

3.1 Reticulados .................... 51

3.2 Teorema de Behrens .................. . . . 60

3.3 Teorema de Stephenson . . . . . . . . . . . . . . . 67

4 Órbitas sob a Ação Regular $\quad 79$

4.1 Teorema de Hirano . . . . . . . . . . . . . . . . . . . . . . 79

4.2 Exemplos . . . . . . . . . . . . . . . . . 86 


\section{Capítulo 1}

\section{Conceitos Básicos}

Neste capítulo serão fixadas terminologia e notações, e serão apresentados, sem demonstração, alguns resultados fundamentais sobre anéis e módulos para referência futura. Para as demonstrações e uma discussão em detalhes dos resultados, referimos o leitor aos livros [LA], [HU], [FD], [RO I], [PS] e [DF].

Em toda a dissertação, o termo ideal significa ideal bilateral. Ideais à esquerda e à direita serão sempre mencionados explicitamente como tais. O termo R-módulo denota um módulo à esquerda sobre um anel $R$; de forma análoga define-se o termo módulo-R. Em todo o texto, o termo anel denotará um anel com unidade. No entanto, em alguns casos diremos "ideal bilateral" ou "anel com unidade" mesmo assim, para efeito de ênfase.

\subsection{Anéis e Módulos Simples}

Definição 1.1 (Anéis e módulos simples). Um anel $R$ é dito simples se $R^{2} \neq 0$ e $R$ não tem ideais bilaterais além de 0 e $R$. Um $R$-módulo $M$ é dito simples se $R M \neq 0$ e $M$ não tem $R$-submódulos além de 0 e $M$. A definição para módulo- $R$ simples é análoga. Um ideal de $R$ pode ser simples como anel ou como $R$-módulo.

Proposição 1.2. Seja $R$ um anel e $M$ um $R$-módulo. São equivalentes:

(i) $M$ é simples.

(ii) $M$ é cíclico e todo elemento não-nulo é um gerador.

(iii) $M \simeq R / I$ para algum ideal à esquerda maximal $I$.

Proposição 1.3. Seja $D$ um anel de divisão e $n$ um inteiro positivo. Então $o$ anel $M_{n}(D)$ é simples. 
Lema 1.4 (Schur). Se $R$ é um anel e $M$ é um $R$-módulo simples, então $\operatorname{End}_{R}(M)$ é um anel de divisão.

Definição 1.5 (anéis e módulos completamente redutíveis). Seja $R$ um anel. Um $R$-módulo $M$ é dito completamente redutivel se e somente se para todo $R$-submódulo $N \subseteq M$ existe um $R$-submódulo $S \subseteq M$ tal que $M=N \oplus S$, ou seja, se todo $R$-submódulo de $M$ é um somando direto. Um anel $R$ é dito completamente redutivel se ${ }_{R} R$ é um módulo completamente redutivel.

Um módulo completamente redutível é também chamado semisimples.

Definição 1.6 (anuladores e ideais primitivos). Sejam $R$ um anel e $M$ um R-módulo. O ideal bilateral

$$
\operatorname{Ann}_{R} M=\{r \in R: r M=0\} \subseteq R
$$

é dito o anulador à esquerda de $M$. Se $N$ é um módulo- $R$,

$$
\operatorname{Ann} N_{R}=\{r \in R: N r=0\} \subseteq R
$$

é dito o anulador à direita de $N$. Se $\operatorname{Ann}_{R} M=0$ (ou $\operatorname{Ann} N_{R}=0$ ) dizemos

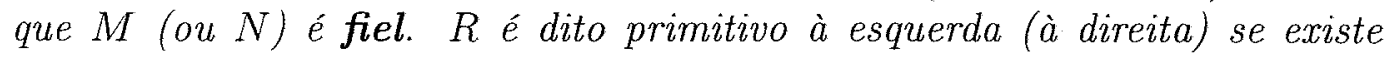
um $R$-módulo (módulo- $R$ ) simples e fiel. Um ideal bilateral $I \subseteq R$ é dito

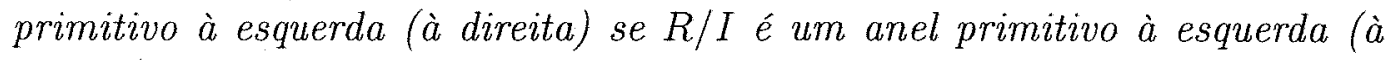
direita).

Proposição 1.7. Sejam $R$ um anel, $M$ um $R$-módulo e $I \subseteq R$ um ideal bilateral contido em $\operatorname{Ann}_{R} M$. Então podemos definir uma estrutura de $R / I$ módulo em $M$ por $(x+I) m=x m, \forall x \in R$ e $\forall m \in M$. Com essa definição, os R-submódulos e os $R / I$-submódulos de $M$ coincidem.

Definição 1.8 (anéis, ideais e módulos indecomponiveis). Um $R$ módulo $M$ é dito indecomponivel se $M$ não tem $R$-submódulos próprios $N_{1}$, $N_{2}$ tais que $M=N_{1} \oplus N_{2}$. Ideais à esquerda indecomponiveis são definidos de forma análoga. Um anel $R$ é dito indecomponível se $R$ não é soma direta de ideais bilaterais não-nulos.

\subsection{Condições de Finitude}

Definição 1.9 (anéis e módulos artinianos). Seja $M$ um $R$-módulo. Dizemos que $M$ satisfaz a condição de cadeia descendente (ou CCD) se toda cadeia de R-submódulos de $M$ da forma

$$
M_{1} \supseteq M_{2} \supseteq \cdots \supseteq M_{n} \supseteq \cdots
$$


termina, ou seja, se existe um $t \in \mathbb{N}$ para o qual $M_{t}=M_{t+i}$ para todo $i \in \mathbb{N}$. Um $R$-módulo satisfazendo a $C C D$ é dito um $R$-módulo artiniano. Define-se analogamente um módulo- $R$ artiniano. Um anel $R$ é dito artiniano à esquerda se e somente se o módulo ${ }_{R} R$ é artiniano, e artiniano à direita se e somente se $R_{R}$ é artiniano.

Definição 1.10 (anéis e módulos noetherianos). Seja $M$ um R-módulo. Dizemos que $M$ satisfaz a condição de cadeia ascendente (ou CCA) se toda cadeia de $R$-submódulos de $M$ da forma

$$
M_{1} \subseteq M_{2} \subseteq \cdots \subseteq M_{n} \subseteq \cdots
$$

termina, ou seja, se existe um $t \in \mathbb{N}$ para o qual $M_{t}=M_{t+i}$ para todo $i \in \mathbb{N}$. Um R-módulo satisfazendo a CCA é dito um $R$-módulo noetheriano. Definese analogamente um módulo- $R$ noetheriano. Um anel $R$ é dito noetheriano à esquerda se e somente se o módulo ${ }_{R} R$ é noetheriano, e noetheriano à direita se e somente se $R_{R}$ é noetheriano.

Teorema 1.11 (condição de mínimo). Seja $R$ um anel e $M$ um $R$-módulo. São equivalentes:

(i) $M$ é artiniano.

(ii) Toda família não-vazia de $R$-submódulos de $M$ contém um elemento minimal.

Teorema 1.12 (condição de máximo). Seja $R$ um anel e $M$ um $R$ módulo. São equivalentes:

(i) $M$ é noetheriano.

(ii) toda familia não-vazia de $R$-submódulos de $M$ contém um elemento maximal.

(iii) todo R-submódulo de $M$ é finitamente gerado.

Lema 1.13 (Zorn). Seja $\mathcal{F}$ uma coleção não-vazia de subconjuntos de um dado conjunto, tal que toda famítia totalmente ordenada de membros de $\mathcal{F}$ tem uma cota superior em $\mathcal{F}$. Então $\mathcal{F}$ contém um elemento maximal.

Como uma aplicação simples do lema de Zorn, temos:

Proposição 1.14. Todo anel $R$ (com 1) contém ideais à esquerda maximais. 
Definição 1.15 (séries de composição). Dizemos que uma cadeia

$$
M=M_{0} \supseteq M_{1} \supseteq \cdots \supseteq M_{n}=0
$$

de R-submódulos de um R-módulo $M$ é uma série de composição de $M$ se todos os quocientes $M_{i} / M_{i+1}$ são R-módulos simples. O número de quocientes $M_{i} / M_{i+1}$ é chamado comprimento da série. Se um módulo tem uma série de composição dizemos que ele é de comprimento finito. Duas séries de composição de $M$ são equivalentes se elas têm o mesmo comprimento e se existe uma bijeção entre os quocientes das duas séries de modo que quocientes correspondentes sejam isomorfos.

Teorema 1.16 (Jordan-Hölder). Todas as séries de composição de um R-módulo $M$ são equivalentes.

Proposição 1.17. Sejam $R$ um anel, $M$ um R-módulo, $A$ um $R$-submódulo de $M$. Então $M$ é artiniano se e somente se $A$ e $M / A$ são artinianos.

Teorema 1.18. Sejam $R$ um anel e $M$ um R-módulo. Então $M$ é de comprimento finito se e somente se $M$ é artiniano e noetheriano.

\subsection{Radical de Jacobson}

Definição 1.19 (quase-regularidade). Dizemos que um elemento $r$ de um anel $R$ é quase-regular à esquerda se $1+r$ tem um inverso à esquerda. Um ideal $I \subseteq R$ é quase-regular à esquerda se todos os seus elementos são quaseregulares à esquerda. Quase-regularidade à direita se define de modo análogo. Dizemos que um elemento $r \in R$ é quase-regular se $1+r \in U(R)$. Um ideal $I \subseteq R$ é quase-regular se todos os seus elementos são quase-regulares.

A proposição abaixo, além de fornecer um outro critério para quaseregularidade, permite generalizar esse conceito para o caso de anéis sem unidade.

Proposição 1.20. Seja $R$ um anel. Se $r \in R$ é quase-regular à esquerda então existe $a \in R$ tal que $a+r-a r=0$. Similarmente, se $r$ é quase-regular à direita então existe $b \in R$ tal que $r+b-r b=0$.

Definição 1.21 (não-geradores). Um elemento $r$ de um anel $R$ é dito não-gerador de $R$ se

$$
A \cup\{r\} \text { é gerador de } R \Longrightarrow A \text { é gerador de } R
$$

para qualquer $A \subseteq R$. 
Definição 1.22 (radical de Jacobson). O radical de Jacobson de um anel $R$, denotado por $J(R)$, é a interseção de todos os anuladores de $R$-módulos simples. Se não existem $R$-módulos simples entâo definimos $J(R)=R$.

Definição 1.23 (ideais nil e ideais nilpotentes). Sejam $R$ um anel $e$ $I \subseteq R$ um ideal. I é chamado de ideal...

(i) nil se para todo $x \in I$ existe um inteiro positivo $n_{x}$ tal que $x^{n_{x}}=0$.

(ii) nil de expoente limitado se existe um inteiro positivo $n$ tal que $x^{n}=0$ para todo $x \in I$.

(iii) nilpotente se existe um inteiro positivo $n$ tal que $I^{n}=0$.

Teorema 1.24 (propriedades do radical). Se $R$ é um anel com unidade, $J(R)$ é um ideal bilateral próprio de $R$ e temos

(i) $J(R)$ é a interseção de todos os ideais à esquerda maximais de $R$.

(ii) $J(R)$ é o único ideal quase-regular à esquerda maximal de $R$.

(iii) $J(R)$ é um ideal quase-regular.

(iv) Se $R$ é artiniano, $J(R)$ é nilpotente.

(v) Se o ideal $I \subseteq R$ é nil, então $I \subseteq J(R)$.

(vi) $J(R / J(R))=0$.

(vii) $J(R)$ é o conjunto de todos os não-geradores de $R$.

(viii) Se $R$ e $S$ são anéis então $J(R \oplus S)=J(R) \oplus J(S)$.

(ix) $J\left(\mathbb{M}_{n}(R)\right)=\mathbb{M}_{n}(J(R))$.

(x) Se $I \subseteq R$ é um ideal e $J(R / I)=0$ então $J(R) \subseteq I$.

\subsection{Anéis Semisimples e Semiprimários}

Definição 1.25. Um anel $R$ é dito semisimples se $J(R)=0$.

Alguns textos chamam de semisimples os anéis $R$ tais que o módulo ${ }_{R} R$

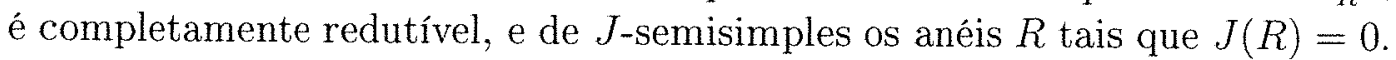
Em geral, ${ }_{R} R$ (respectivamente, $R_{R}$ ) é completamente redutível se, e somente se, $J(R)=0$ e $R$ é artiniano. 
Teorema 1.26. Seja $R$ um anel. São equivalentes:

(i) $R$ é artiniano semisimples.

(ii) ${ }_{R} R$ é completamente redutível.

(iii) $R$ é uma soma direta finita de ideais à esquerda minimais.

Proposição 1.27. Seja $R=\bigoplus_{i=1}^{t} L_{i}$ um anel artiniano semisimples, decomposto em uma soma direta finita de ideais à esquerda minimais. Então todo $R$-módulo simples é isomorfo a um dos ideais à esquerda $L_{i}$.

Proposição 1.28. Seja $L$ um ideal à esquerda minimal de um anel artiniano semisimples $R$ e seja $A$ a soma (direta) de todos os ideais à esquerda de $R$ isomorfos a L. Então $A$ é um ideal bilateral minimal de $R$.

Os ideais bilaterais minimais da proposição acima são denominadas as componentes simples do anel artiniano semisimples $R$. Em vista das proposições (1.27) e (1.28) acima, uma componente simples determina uma classe de isomorfismos de $R$-módulos simples. É claro que um anel artiniano simples $R$ tem uma única componente simples; portanto, todos os ideais à esquerda minimais de $R$ (e portanto todos os $R$-módulos simples) são isomorfos entre si.

Teorema 1.29 (Wedderburn). Seja $R$ um anel não-nulo e artiniano à esquerda. São equivalentes:

(i) $R$ é simples.

(ii) $R$ é primitivo à esquerda.

(iii) $R \simeq M_{n}(D)$ para algum anel de divisão $D$ e algum inteiro positivo $n$.

Teorema 1.30 (Wedderburn-Artin). Seja $R$ um anel.

São equivalentes:

(i) $R$ é artiniano e semisimples.

(ii) Existem um inteiro positivo $t$, anéis de divisão $D_{1}, \ldots, D_{t}$ e inteiros positivos $n_{1}, \ldots, n_{t}$ tais que $R \simeq \mathbb{M}_{n_{1}}\left(D_{1}\right) \oplus \cdots \oplus \mathbb{M}_{n_{t}}\left(D_{t}\right)$.

Teorema 1.31. Seja $R$ um anel semisimples artiniano. São equivalentes:

(i) $R$ não contém ideais nilpotentes não-nulos.

(ii) $R$ não contém ideais à esquerda nilpotentes não-nulos. 
Definição 1.32 (anéis semiprimários). Um anel $R$ é dito semiprimário se $J=J(R)$ é nilpotente e $R / J$ é artiniano.

Teorema 1.33 (Levitzki). Se $R$ é um anel noetheriano à esquerda, então todo ideal nil de $R$ (à esquerda, à direita ou bilateral) é nilpotente.

Teorema 1.34 (Hopkins-Levitzki). Seja $R$ um anel semiprimário. Para todo R-módulo ${ }_{R} M$ são equivalentes:

(i) $M$ é noetheriano.

(ii) $M$ é artiniano.

(iii) $M$ tem uma série de composição.

Em particular, um anel é artiniano à esquerda se e somente se é semiprimário e noetheriano à esquerda.

\subsection{Idempotentes}

Definição 1.35 (idempotentes). Seja $R$ um anel. Um elemento $e \in R$ é chamado idempotente se $e^{2}=e$.

Teorema 1.36. Seja $R$ um anel. São equivalentes:

(i) $R$ é artiniano semisimples;

(ii) todo ideal à esquerda $I \subseteq R$ é da forma $I=$ Re para algum idempotente $e \in R$.

Teorema 1.37. Seja $R$ um anel semisimples artiniano e $R=\bigoplus_{i=1}^{t} L_{i}$ uma decomposição de $R$ em uma soma direta finita de ideais à esquerda minimais. Então existe uma familia $\left\{e_{1}, \ldots, e_{t}\right\}$ de elementos não-nulos de $R$ tais que $L_{i}=R e_{i}, 1 \leqslant i \leqslant t, e$

(i) $e_{i}$ é um idempotente, $1 \leqslant i \leqslant t$;

(ii) $e_{i} e_{j}=0$ se $i \neq j$;

(iii) $1=e_{1}+\cdots+e_{t}$;

(iv) $e_{i}$ não pode ser escrito como $e_{i}=e_{i}^{\prime}+e_{i}^{\prime \prime}$, sendo $e_{i}^{\prime}$, $e_{i}^{\prime \prime}$ idempotentes não-nulos tais que $e_{i}^{\prime} e_{i}^{\prime \prime}=0,1 \leqslant i \leqslant t$. 
Reciprocamente, se existe uma família de idempotentes $\left\{e_{1}, \ldots, e_{t}\right\}$ em $R$ satisfazendo as condições acima, então os ideais à esquerda $L_{i}=\operatorname{Re}_{i}$ são minimais e $R=\bigoplus_{i=1}^{t} R e_{i}$.

Definição 1.38. Seja $R$ um anel. Uma família de idempotentes $\left\{e_{1}, \ldots, e_{t}\right\}$ satisfazendo as condiçôes (i), (ii) e (iii) do teorema acima é chamada uma familia completa de idempotentes ortogonais. Se a condição (iv) acima também é satisfeita, os idempotentes são ditos primitivos.

Lema 1.39 (Brauer). Seja $R$ um anel qualquer e $L \subseteq R$ um ideal à esquerda minimal. Então $L^{2}=0$ ou $L=$ Re para algum idempotente primitivo nãonulo e $\in R$.

Teorema 1.40. Seja $R=\bigoplus_{i=1}^{t} A_{i}$ uma decomposição do anel artiniano semisimples $R$ como uma soma direta finita de ideais bilaterais minimais. Então existe uma família completa de idempotentes ortogonais $\left\{e_{1}, \ldots, e_{t}\right\}$, centrais em $R$, tais que $A_{i}=R_{i}$ e cada e é o elemento identidade de $A_{i}$, $1 \leqslant i \leqslant t$. Nesse caso, $R=\bigoplus_{i=1}^{t} R e_{i}$.

\subsection{Módulos}

Teorema 1.41 (Krull-Schmidt). Se

$$
M=M_{1} \oplus \cdots \oplus M_{m}=N_{1} \oplus \cdots \oplus N_{n}
$$

são decomposições do módulo $M$ em soma direta de módulos indecomponíveis, então $m=n$ e, renumerando os indices se necessário, temos $M_{i} \simeq N_{i}$ para todo $i, 1 \leqslant i \leqslant n$.

Lema 1.42 (Nakayama). Seja $R$ um anel. Para todo ideal à esquerda $L \subseteq R$, são equivalentes:

(i) $L \subseteq J(R)$.

(ii) Para todo $R$-módulo $M$ finitamente gerado, $L M=M \Longrightarrow M=0$.

(iii) Para quaisquer $R$-módulos $M, N$ tais que $N \subseteq M$ e $M / N$ é finitamente gerado, temos $N+L M=M \Longrightarrow N=M$.

Definição 1.43 (seqüências exatas). Sejam $R$ um anel e $X, Y, Z$ módulos $\grave{a}$ esquerda sobre $R$.

1. Dizemos que o par de homomorfismos $X \stackrel{\alpha}{\longrightarrow} Y \stackrel{\beta}{\longrightarrow} Z$ é exato em $Y$ se $\operatorname{im} \alpha=\operatorname{ker} \beta$. 
2. Se $\ldots, X_{n-1}, X_{n}, X_{n+1}, \ldots$ são $R$-módulos dizemos que uma seqüência de $R$-homomorfismos $\cdots \rightarrow X_{n-1} \rightarrow X_{n} \rightarrow X_{n+1} \rightarrow \cdots$ é exata se qualquer par de homomorfismos consecutivos for exato.

Proposição 1.44. Seja $R$ um anel e $P$ um $R$-módulo. São equivalentes:

(i) Para quaisquer $R$-módulos $M, N$, se a seqüência $M \stackrel{\varphi}{\longrightarrow} N \longrightarrow 0$ é exata e $f: P \rightarrow N$ é um R-homomorfismo, então existe um (único) $R$-homomorfismo $F: P \rightarrow M$ tal que o diagrama abaixo comuta:

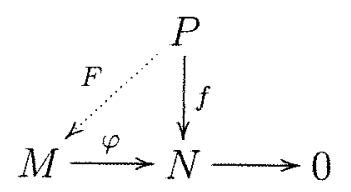

(ii) Toda seqüência exata $0 \rightarrow L \rightarrow M \rightarrow P \rightarrow 0$ de $R$-módulos cinde.

(iii) $P$ é somando direto de algum $R$-módulo livre.

Definição 1.45 (módulos projetivos). Seja $R$ um anel. Um módulo ${ }_{R} M$ $e ́$ dito projetivo se satisfaz as condiçôes equivalentes da proposição (1.44).

Proposição 1.46. Toda soma direta de $R$-módulos projetivos é um $R$-módulo projetivo.

Lema 1.47. Sejam $R$ um anel, $I \subseteq R$ um ideal e $P$ um $R$-módulo. Se $P$ é projetivo como $R$-módulo então $P / I P$ é projetivo como $R / I$-módulo.

Proposição 1.48. Seja $R$ um anel.

(i) Se $0 \rightarrow L \rightarrow M \rightarrow N \rightarrow 0$ é uma seqüência exata de $R$-módulos e $D$ é um módulo- $R$, então $D \otimes_{R} L \rightarrow D \otimes_{R} M \rightarrow D \otimes_{R} N \rightarrow 0$ é uma seqüência exata de grupos abelianos.

(ii) Se $0 \rightarrow L \rightarrow M \rightarrow N \rightarrow 0$ é uma seqüência exata de módulos- $R$ e $D$ é um R-módulo, então $L \otimes_{R} D \rightarrow M \otimes_{R} D \rightarrow N \otimes_{R} D \rightarrow 0$ é uma seqüência exata de grupos abelianos.

Corolário 1.49. Seja $R$ um anel e $D$ um módulo-R. São equivalentes:

(i) Se $0 \rightarrow L \rightarrow M \rightarrow N \rightarrow 0$ é uma seqüência exata de $R$-módulos, então $0 \rightarrow D \otimes_{R} L \rightarrow D \otimes_{R} M \rightarrow D \otimes_{R} N \rightarrow 0$ é uma seqüência exata de grupos abelianos.

(ii) Se $L, M$ são $R$-módulos e $\varphi: L \rightarrow M$ é um monomorfismo, a aplicação induzida $1 \otimes_{R} \varphi: D \otimes_{R} L \rightarrow D \otimes_{R} M$ é um monomorfismo de grupos abelianos. 
Definição 1.50 (módulos planos). Seja $R$ um anel. Um módulo $M_{R}$ é dito plano se satisfaz as condições equivalentes do corolário (1.49).

O corolário (1.49) é conseqüência imediata da primeira parte da proposição (1.48). De modo análogo, é possível enunciar uma versão simétrica do corolário (1.49), conseqüência da segunda parte da proposição (1.48), ao permutarmos esquerda por direita. Sendo assim, podemos definir a noção de $R$-módulo plano da mesma forma. Ambas as definições de módulo plano serão usadas na seção 2.4 do capítulo 2 .

Proposição 1.51. Seja $R$ um anel. Todo módulo livre sobre $R$ é plano, e todo módulo projetivo sobre $R$ é plano. 


\section{Capítulo 2}

\section{Anéis Semiperfeitos e Perfeitos}

O objetivo deste capítulo é descrever duas classes de anéis: a dos anéis semiperfeitos e a dos anéis perfeitos. Hyman Bass, em seu importante artigo de 1960 [BA], os concebeu como generalizações de anéis semiprimários. Em geral, se $R$ é um anel temos a seguinte cadeia de implicações:

$R$ é artiniano $\Rightarrow R$ é semiprimário $\Rightarrow R$ é perfeito $\Rightarrow R$ é semiperfeito.

Todos essas classes de anéis são exemplos de anéis semilocais, ou seja, anéis $R$ tais que o quociente $R / J(R)$ é artiniano. Veremos também que as denominações acima têm razão de ser, ou seja, há exemplos de anéis semilocais que não são semiperfeitos, semiperfeitos que não são perfeitos, perfeitos que não são semiprimários, e semiprimários que não são artinianos.

Após uma breve exposição sobre anéis locais na seção 2.1, o conceito de anel semiperfeito é desenvolvido na seção 2.2 , e anéis perfeitos são tratados na seção 2.3. A abordagem é estrutural, utilizando idempotentes. A seguir serão desenvolvidas caracterizações homológicas de anéis semiperfeitos e perfeitos na seção 2.4, culminando no teorema de Bass (2.58). Na seção 2.5, serão apresentados alguns exemplos, que ilustrarão a cadeia de implicações acima. A exposição segue, principalmente, os livros de Behrens [BE] e Lam [LA].

\subsection{Anéis Locais}

Em Álgebra Comutativa, um anel local é definido como um anel não-nulo que tem um único ideal maximal. A generalização natural para o caso nãocomutativo é definir anel local como um anel não-nulo contendo um único ideal à esquerda maximal, ou, de forma equivalente, um único ideal à direita maximal. 
Começaremos com um teorema de caracterização, que oferecerá outros critérios que definem anéis locais.

Teorema 2.1 (caracterização de anéis locais). Para todo anel não-nulo $R$ com radical $J=J(R)$, são equivalentes as seguintes afirmações:

(i) $R$ tem um único ideal à esquerda maximal; esse ideal é $J$.

(ii) $R / J$ é um anel de divisão.

(iii) $R \backslash U(R)=J$.

(iv) Se $a+b \in U(R)$ então $a \in U(R)$ ou $b \in U(R)$.

Se qualquer uma dessas condições é satisfeita, dizemos que o anel $R$ é local.

\section{Prova.}

$($ ii $) \Rightarrow(i)$ Todo ideal à esquerda maximal $L \subsetneq R$ contém o radical $J(R)$. Se $R / J$ é um anel de divisão então $R / J$ é um $R$-módulo simples, donde $J$ é um ideal à esquerda maximal de $R$. Por maximalidade de $L$ temos que $L=J$, ou seja, $J$ é o único ideal à esquerda maximal de $R$.

$(i) \Rightarrow$ (ii) Se $R$ tem um único ideal à esquerda maximal, então $J(R)$ é essē ideal à esquerda maximal, pela definição de radical. Daí $R / J$ é um anel de divisão.

(ii) $\Rightarrow$ (iii) Por hipótese, se $a \notin J(R)$ então $a+J=\bar{a}$ é um elemento invertível de $R / J$, ou seja, existe $b \in R$ tal que $\bar{a} \bar{b}=\bar{b} \bar{a}=\overline{1}$. Então $a b-1 \in J$ e $b a-1 \in J$, donde $a b, b a \in U(R)$ pelo teorema (1.24), e portanto $a \in U(R)$. É claro que $J \subseteq R \backslash U(R)$. Provamos, assim, que $J=R \backslash U(R)$.

$($ iii $) \Rightarrow($ iv) Se $a \notin U(R)$ e $b \notin U(R)$ então $a, b \in J$ por hipótese, logo $a+b \in J$, ou seja, $a+b \notin U(R)$.

$(i v) \Rightarrow(i i)$ Tome $a \notin J$. Pelo teorema (1.24), J está contido em todo ideal à esquerda maximal de $R$; assim, existe um ideal à esquerda maximal $L \subsetneq R$ tal que $a \notin L$. Por maximalidade de $L$ temos que $L+R a=R$, donde $\ell+r a=1$ para alguns $\ell \in L, r \in R$. Como $\ell \notin U(R)$, pela hipótese $(i v)$ temos que $r a \in U(R)$. Então $\bar{a}$ possui um inverso à esquerda em $\bar{R}=R / J$, ou seja, $\bar{R} \backslash\{\overline{0}\}$ é um grupo multiplicativo; logo, $\bar{R}$ é um anel de divisão.

O radical $J$ de um anel $R$ pode ser definido como a interseção de todos os ideais à direita maximais de $R$; sendo assim, imitando a prova do item (i) $\Rightarrow$ (ii) acima, não é difícil ver que um anel é local se e somente se tem um único ideal à direita maximal. No entanto, não precisaremos desse fato na seqüência.

A próxima proposição nos fornece algumas condições necessárias (mas não suficientes) para que um anel seja local. 
Proposição 2.2. Seja $R$ um anel local. Então:

(i) $R$ tem um único ideal maximal.

(ii) Se $a \in R$ admite um inverso à esquerda, então $a \in U(R)$.

(iii) Os únicos idempotentes de $R$ são os triviais 0 e 1 .

\section{Prova.}

(i) Um ideal maximal $I \subsetneq R$ não contém elementos invertíveis, logo

$$
I \subseteq R \backslash U(R)=J
$$

donde $I=J$ pela maximalidade de $I$. Daí $J$ é o único ideal maximal de $R$.

(ii) Se $a \in R$ admite um inverso à esquerda então $a \notin J$, donde $a \in U(R)$ pelo teorema $(2.1)$ (iii).

(iii) Seja $e \in R$ um idempotente e seja $f=1-e$; pelo teorema (2.1)(iv), temos que $e \in U(R)$ ou $f \in U(R)$. Como ef $=0$, temos $e=0$ ou $f=0$.

Já vimos a definição de anéis e módulos indecomponiveis em (1.8). Segue facilmente que um $R$-módulo é indecomponível se, e somente se, o anel de $R$-endomorfismos $\operatorname{End}_{R}(M)$ não possui projeções além de 0 e 1, ou seja, só possui idempotentes triviais. De fato, se há $R$-submódulos $N_{1}, N_{2} \subseteq M$ tais que $M=N_{1} \oplus N_{2}$ então as projeções naturais $\pi_{1}: M \rightarrow N_{1}$ e $\pi_{2}: M \rightarrow N_{2}$ são idempotentes não-triviais de $\operatorname{End}_{R}(M) ;$ reciprocamente, se $\theta \in \operatorname{End}_{R}(M)$ é um idempotente não-trivial, temos então que $M=\operatorname{im} \theta \oplus \operatorname{im}(1-\theta)$, sendo 1 a identidade de $\operatorname{End}_{R}(M)$.

A discussão acima motiva a seguinte definição:

Definição 2.3 (módulos fortemente indecomponíveis). Um R-módulo não-nulo $M$ é dito fortemente indecomponivel se $\operatorname{End}_{R}(M)$ é um anel local.

\section{Exemplo 2.4.}

Todo anel de divisão é um anel local. Logo, todo módulo simples ${ }_{R} M$ é fortemente indecomponível, uma vez que $\operatorname{End}_{R}(M)$ é um anel de divisão,

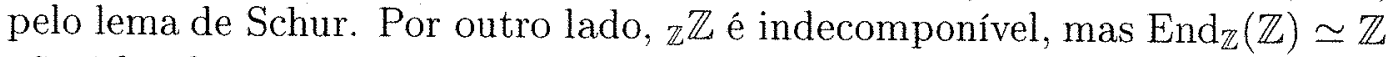
não é local, portanto $\mathbb{Z} \mathbb{Z}$ não é fortemente indecomponível.

Lema 2.5. Seja $R$ um anel. Se e, $f \in R$ são idempotentes e $M$ é um $R$ módulo, então $\operatorname{Hom}_{R}(R e, M) \simeq e M$ como grupos abelianos. Em particular, $\mathrm{Hom}_{R}(R e, R f) \simeq e R f$ como grupos abelianos. 
Prova. Dado um $R$-homomorfismo $\theta: R e \rightarrow M$ qualquer, defina $m=\theta(e)$. Então $e m=e \theta(e)=\theta\left(e^{2}\right)=\theta(e)=m, \log o m=e m \in e M$. Podemos definir $\Phi: \operatorname{Hom}_{R}(R e, M) \rightarrow e M$ pondo $\Phi(\theta)=\theta(e)$, e é fácil ver que $\Phi$ é um monomorfismo de grupos. Para mostrar que $\Phi$ é um epimorfismo, considere $m \in e M$ arbitrário e defina $\theta: R e \rightarrow M$ pondo $\theta(r e)=r m$ para todo $r \in R$. É fácil ver que $\theta$ está bem definida e é um $R$-homomorfismo. Então $\Phi$ é um epimorfismo, uma vez que $\Phi(\theta)=\theta(e)=m$. Para ver que $\operatorname{Hom}_{R}(R e, R f) \simeq e R f$, basta considerar $M=R f$.

Uma conseqüência disso é a seguinte caracterização de ideais à esquerda fortemente indecomponíveis:

Corolário 2.6. Para todo idempotente e de um anel $R$, são equivalentes:

(i) Re é fortemente indecomponível como R-módulo.

(ii) eRe é um anel local.

Prova. Basta tomar $e=f$ no lema (2.5). Note que, nesse caso, o isomorfismo $\Psi: \operatorname{Hom}_{R}(R e, R e) \rightarrow e R e$ é também um isomorfismo de anéis.

Corolário 2.7. Sejam $R$ um anel e $e \in R$ um idempotente. Então eRe e o anel de endomorfismos (à direita) $\operatorname{End}(e R)_{R}$ são isomorfos como anéis.

Prova. Adaptando a prova do lema (2.5) para o caso em que $M$ é um módulo à direita sobre $R$, é fácil concluir que $\Psi: \operatorname{End}(e R)_{R} \rightarrow$ eRe é um isomorfismo de grupos abelianos definido por $\Psi(\alpha)=\alpha(e)$ para todo $\alpha \in \operatorname{End}(e R)_{R}$. Observe agora que para todos $\alpha, \beta \in \operatorname{End}(e R)_{R}$ temos

$$
\Psi(\alpha \circ \beta)=\alpha(\beta(e))=\alpha(e \cdot \beta(e))=\alpha(e) \beta(e)=\Psi(\alpha) \Psi(\beta),
$$

logo $\Psi$ é um isomorfismo de anéis.

\subsection{Anéis Semiperfeitos}

Definição 2.8 (anéis semiperfeitos). Um anel $R$ com radical $J$ é dito semiperfeito se

(i) $R / J$ é artiniano (um anel com essa propriedade é chamado semilocal).

(ii) A todo idempotente $u+J \in R / J$ corresponde um idempotente $e \in R$ tal que $e+J=u+J$. 
Se vale a segunda condição, dizemos que os idempotentes do quociente podem ser levantados, ou que $J$ é "idempotent-lifting".

A classe dos anéis semilocais contém propriamente a classe dos anéis semiperfeitos, como será visto mais adiante no exemplo (2.62).

A proposição abaixo estabelece que os idempotentes de $R / I$ podem ser levantados sempre que $I \subseteq R$ for um ideal nil.

Proposição 2.9. Seja I um ideal nil do anel $R$ e $\ddot{R}=R / I$. Se $\bar{a} \in \bar{R}$ é um idempotente, então existe um idempotente $e \in R$ tal que $\bar{e}=\bar{a}$.

Prova. Defina $b=1-a$. Temos que $a b=b a=a-a^{2} \in I$, de modo que $(a b)^{n}=a^{n} b^{n}=0$ para algum inteiro positivo $n$. Pelo teorema binomial,

$$
\begin{aligned}
1 & =(a+b)^{2 n} \\
& =a^{2 n}+\left(\begin{array}{c}
2 n \\
1
\end{array}\right) a^{2 n-1} b+\cdots+\left(\begin{array}{c}
2 n \\
n
\end{array}\right) a^{n} b^{n}+\left(\begin{array}{c}
2 n \\
n+1
\end{array}\right) a^{n-1} b^{n+1}+\cdots+b^{2 n}
\end{aligned}
$$

Defina agora

$$
\begin{aligned}
& e=a^{2 n}+\left(\begin{array}{c}
2 n \\
1
\end{array}\right) a^{2 n-1} b+\cdots+\left(\begin{array}{c}
2 n \\
n
\end{array}\right) a^{n} b^{n}, \\
& f=\left(\begin{array}{c}
2 n \\
n+1
\end{array}\right) a^{n-1} b^{n+1}+\cdots+b^{2 n}
\end{aligned}
$$

Daí $e+f=1$ e é fácil ver que $e f=0$. Então $e$ é um idempotente de $R$, uma vez que $e=e(e+f)=e^{2}$. Como $a b \in I$, temos $e \equiv a^{2 n} \equiv a(\bmod I)$.

Como ficará claro no exemplo (2.63), a condição acima é uma condição suficiente, mas não necessária, para que idempotentes possam ser levantados.

Corolário 2.10. Todo anel artiniano é semiperfeito.

Prova. Se $R$ é artiniano, então $R / J$ é artiniano e $J=J(R)$ é nilpotente. Pela proposição (2.9), idempotentes de $R / J$ podem ser levantados.

A partir de agora, nosso objetivo é mostrar que, se $R$ é semiperfeito, então o processo de levantar idempotentes de $R / J(R)$ ou projetá-los de $R$ sobre $R / J(R)$ preserva famílias completas de idempotentes ortogonais primitivos.

Para começar, apresentamos dois lemas técnicos e um corolário que são independentes do resto do capítulo, pois não precisam da hipótese do anel $R$ ser semiperfeito. 
Lema 2.11. Sejam $R$ um anel e e, $f \in R$ idempotentes. Então Re e $R f$ são isomorfos como $R$-módulos se, e somente se, existem elementos $u, v \in R$ tais que $u v=e, v u=f$.

Prova. Suponha que exista um isomorfismo $\varphi \in \operatorname{Hom}_{R}(R e, R f)$. Então existe $u^{\prime} \in R$ tal que $\varphi(e)=u^{\prime} f$; como $e \varphi(e)=\varphi(e)=e u^{\prime} f$, temos em geral que $\varphi(x e)=x e u^{\prime} f$ para todo $x \in R$. Seja $\psi=\varphi^{-1} \in \operatorname{Hom}_{R}(R f, R e)$ o seu inverso; similarmente, existe $v^{\prime} \in R$ tal que $\psi(y f)=y f v^{\prime} e$ para todo $y \in R$. Então

$$
\begin{aligned}
\psi \circ \varphi(e)=e & \Longrightarrow \quad e u^{\prime} f v^{\prime} e=e ; \\
\varphi \circ \psi(f)=f & \Longrightarrow f v^{\prime} e u^{\prime} f=f .
\end{aligned}
$$

Definindo $u=e u^{\prime} f$ e $v=f v^{\prime} e$, temos $e=u v$ e $f=v u$.

Reciprocamente, suponha que $e=u v$ e $f=v u$ para alguns $u, v \in R$. Então

$$
\begin{gathered}
x e u=x(u v) u=x u(v u)=x u f \in R f \\
y f v=y(v u) v=y v(u v)=y v e \in R e
\end{gathered}
$$

para todos $x, y \in R$. Daí podemos definir as aplicações $\varphi \in \operatorname{Hom}_{R}(R e, R f) \mathrm{e}$ $\psi \in \operatorname{Hom}_{R}(R f, R e)$ pondo $\varphi(x e)=x e u$ e $\psi(y f)=y f v$ para todos $x, y \in R$. Uma conta simples mostra que $\psi \circ \varphi(x e)=x e$ e $\varphi \circ \psi(y f)=y f$ para todos $x, y \in R, \log 0 \varphi$ e $\psi$ são isomorfismos.

Lema 2.12. Sejam $R$ um anel, e, $f \in R$ idempotentes e $J=J(R)$. Se existem elementos $u^{\prime}, v^{\prime} \in R$ tais que

$$
u^{\prime} v^{\prime} \equiv e(\bmod J) \quad e \quad v^{\prime} u^{\prime} \equiv f(\bmod J),
$$

então existem $u, v \in R$ tais que $e=u v$ e $f=v u$.

Prova. Observe que

$$
\begin{gathered}
f v^{\prime} \equiv v^{\prime} u^{\prime} v^{\prime} \equiv v^{\prime} e(\bmod J) \\
e u^{\prime} \equiv u^{\prime} v^{\prime} u^{\prime} \equiv u^{\prime} f(\bmod J)
\end{gathered}
$$

$\log 0$

$$
e u^{\prime} f v^{\prime} \equiv u^{\prime} f v^{\prime} \equiv u^{\prime} v^{\prime} e \equiv e^{2} \equiv e(\bmod J) .
$$

Daí $x=e-e u^{\prime} f v^{\prime} \in J$ e, como $x=e x$, temos

$$
e u^{\prime} f v^{\prime}=e-x=e(1-x)
$$


Como $x \in J$, existe $y \in J$ tal que $(1-x)(1-y)=1$. Defina

$$
v=f v^{\prime}(1-y) \quad \text { e } \quad u=e u^{\prime} f
$$

segue que

$$
e=e(1-x)(1-y)=e u^{\prime} f \cdot f v^{\prime}(1-y)=u v .
$$

Temos também $(v u)^{2}=v u v u=v e u=v u$. Isso implica que

$$
(f-v u)^{2}=f-f v u-v u f+v u=f-v u .
$$

Mas como

$$
v u=f v^{\prime}(1-y) e u^{\prime} f \equiv f v^{\prime} e u^{\prime} f \equiv f v^{\prime} u^{\prime} f \equiv f^{3} \equiv f(\bmod J),
$$

o idempotente $f$-vu pertence ao radical. Pela proposição (2.14), $f=v u$.

Corolário 2.13. Sejam $R$ um anel, e, $f \in R$ idempotentes, $J=J(R)$ seu radical e $\bar{R}=R / J$. Os ideais à esquerda Re e Rf são isomorfos como $R$-módulos se e somente se $\bar{R} \bar{e}$ e $\bar{R} \bar{f}$ são isomorfos como $\bar{R}$-módulos.

Prova. Se $R e \simeq R f$, pelo lema (2.11) existem elementos $u, v \in R$ tais que $u v=e, v u=f$. Então $\bar{u} \bar{v}=\bar{e}, \bar{v} \bar{u}=\bar{f}$, donde $\bar{R} \bar{e} \simeq \bar{R} \bar{f}$ pelo lema (2.11). Reciprocamente, se $\bar{R} \bar{e} \simeq \bar{R} \bar{f}$ então existem elementos $\bar{u}, \bar{v} \in \bar{R}$ tais que $\bar{u} \bar{v}=\bar{e}, \bar{v} \bar{u}=\bar{f}$. Pelo lema (2.12) temos $u v=e, v u=f$, logo $R e \simeq R f$.

A seguir, veremos que o único idempotente no radical de um anel é o 0 , ou seja, não há idempotentes próprios no radical.

Proposição 2.14. Seja $R$ um anel. Se e $\in J(R)$ é um idempotente, $e=0$.

Prova. Seja $g=g^{2} \in J(R)$ um idempotente não-nulo. Como $J(R)$ é um ideal quase-regular (1.19 e 1.24), existe $a \in R$ tal que $a+g-a g=0$ pela proposição (1.20); multiplicando por $g$ à direita obtemos $g^{2}=g=0$, uma contradição.

Seja $J=J(R)$ o radical do anel $R$. Se $R / J$ é artiniano então $R / J$ é completamente redutível. Nesse caso $\bar{R}=R / J=\bar{R} \bar{u}_{1} \oplus \cdots \oplus \bar{R} \bar{u}_{t}$, sendo $\left\{\bar{u}_{1}, \ldots, \bar{u}_{t}\right\}$ uma família completa de idempotentes ortogonais primitivos em $R / J$. Se pudermos levantar $\left\{\bar{u}_{1}, \ldots, \bar{u}_{t}\right\}$ a uma família completa $\left\{e_{1}, \ldots, e_{t}\right\}$ de idempotentes ortogonais em $R$ tais que $\bar{e}_{i}=\bar{u}_{i}, 1 \leqslant i \leqslant t$, então os idempotentes $e_{i}$ também serão primitivos. De fato, se $e_{i}=f_{i}+g_{i}, f_{i}$ e $g_{i}$ idempotentes ortogonais não-nulos, então $\bar{e}_{i}=\bar{u}_{i}=\bar{f}_{i}+\bar{g}_{i}$; mas $\bar{f}_{i}$ e $\bar{g}_{i}$ não são nulos, pois não há idempotentes não-nulos no radical pela proposição (2.14). Isso contradiz o fato de que os idempotentes $\bar{u}_{i}$ são primitivos.

A próxima proposição nos dará a recíproca desse fato. 
Proposição 2.15. Sejam $R$ um anel semiperfeito, $e \in R$ um idempotente $e$ $J=J(R)$. Então $\bar{e} \in \bar{R}=R / J(R)$ é primitivo se, e somente se, $e \in R$ é primitivo.

Prova. Se $\bar{e} \in \bar{R}$ é primitivo, pela discussão precedente já sabemos que $e \in R$ é primitivo. Reciprocamente, suponha que $\bar{e}$ não é primitivo, ou seja, suponha que $\bar{e}=x+y$ é uma decomposição de $\bar{e}$ em uma soma de idempotentes ortogonais não-nulos $x, y \in \bar{R}$. Como $R$ é semiperfeito, existem idempotentes $\alpha, \beta \in R$ tais que $\bar{\alpha}=x$ e $\bar{\beta}=y$. Como $x y=\overline{0}$, então $\bar{\alpha} \bar{\beta}=\bar{\beta} \bar{\alpha}=\overline{0}$. Precisamos mostrar que e não é primitivo.

Afirmação: Existe um idempotente $\beta^{\prime} \in R$, ortogonal a $\alpha$, tal que $\bar{\beta}^{\prime}=\bar{\beta}$.

Provemos essa afirmação. Como $\beta \alpha \in J$, então $1-\beta \alpha \in U(R)$. Defina

$$
\beta_{0}=(1-\beta \alpha)^{-1} \beta(1-\beta \alpha) ;
$$

é claro que $\beta_{0}$ é idempotente e que $\bar{\beta}_{0}=\bar{\beta}$. Um cálculo simples nos mostra que $\beta_{0} \alpha=0$, mas $\alpha \beta_{0}$ pode ser diferente de zero. Defina então

$$
\beta^{\prime}=(1-\alpha) \beta_{0} .
$$

Como $\bar{\alpha} \bar{\beta}=\bar{\alpha} \bar{\beta}_{0}=\overline{0}$, temos $\bar{\beta}^{\prime}=\bar{\beta}_{0}=\bar{\beta}$. Portanto, temos

$$
\beta^{\prime} \alpha=(1-\alpha) \beta_{0} \alpha=0 \quad \text { e } \quad \alpha \beta^{\prime}=\left(\alpha-\alpha^{2}\right) \beta_{0}=0 .
$$

Um cálculo simples mostra que $\beta^{\prime}$ também é idempotente, logo a afirmação está provada.

Defina $e^{\prime}=\alpha+\beta^{\prime}$. É fácil ver que $e^{\prime}$ é idempotente e, como $\alpha$ e $\beta^{\prime}$ são ortogonais e diferentes de zero, $e^{\prime}$ não é primitivo. Passando ao quociente, podemos escrever $\bar{e}^{\prime}=\bar{\alpha}+\bar{\beta}^{\prime}=\bar{e}$. Logo, pelo corolário (2.13), temos que $R e \simeq R e^{\prime}$ como $R$-módulos. Então

$$
R e \simeq R e^{\prime}=R \alpha \oplus R \beta^{\prime},
$$

portanto e não é primitivo.

No próximo lema veremos que se $R$ é um anel, idempotentes de $R / I$ podem ser levantados e temos dois idempotentes ortogonais em $R / I$, então eles podem ser levantados a dois idempotentes ortogonais de $R$.

Lema 2.16. Sejam $R$ um anel $e I \subseteq J=J(R)$ um ideal tal que idempotentes de $R / I$ possam ser levantados. Sejam $g \in R$ e $u+I=\bar{u} \in R / I$ idempotentes não-nulos. Se $\bar{u} \bar{g}=\bar{g} \bar{u}=\tilde{0}$, existe um idempotente $e \in R$ tal que $\bar{e}=\bar{u} e$ $e g=g e=0$. 
Prova. Como idempotentes de $\bar{R}=R / I$ podem ser levantados, existe um idempotente $f \in R$ tal que $\bar{f}=\bar{u}$. Logo $\bar{f} \bar{g}=\bar{g} \bar{f}=\overline{0}$, donde $f g \in J$ e portanto $1-f g$ é invertível. Defina $f^{\prime}=(1-f g)^{-1} f(1-f g)$; segue que $f^{\prime}$ também é um idempotente, e que

$$
f(1-f g) g=f g-f^{2} g^{2}=0 \Longrightarrow f^{\prime} g=0 .
$$

Note ainda que $f^{\prime} \equiv(1-f g) f^{\prime}=f(1-f g) \equiv f(\bmod I)$. Defina agora $e=(1-g) f^{\prime}$. Então $\bar{e}=(\overline{1}-\bar{g}) \bar{f}^{\prime}=\bar{f}-\bar{g} \bar{f}=\bar{u}, g e=e g=0 \mathrm{e}$ $e^{2}=e(1-g) f^{\prime}=e f^{\prime}=(1-g) f^{\prime}=e$.

Esse fato pode ser estendido a um número contável de idempotentes, como será feito na proposição a seguir.

Proposição 2.17. Sejam $R$ um anel e $I \subseteq J(R)$ um ideal de $R$ tal que os idempotentes de $R / I$ possam ser levantados. Se $\left\{\bar{u}_{1}, \ldots, \bar{u}_{t}, \ldots\right\}$ é uma família de idempotentes não-nulos ortogonais em $R / I$, então existe uma familia ortogonal $\left\{e_{1}, \ldots, e_{t}, \ldots\right\}$ de idempotentes em $R$ tais que $\bar{e}_{i}=\bar{u}_{i}$ para todo inteiro positivo $i$.

Prova. Por indução em $i$. Suponha que $\bar{u}_{1}, \ldots, \bar{u}_{t-1}$ já foram levantados a idempotentes ortogonais $e_{1}, \ldots, e_{t-1}$ em $R$. Defina $g=e_{1}+\cdots+e_{t-1}$. Então

$$
u_{t} g=\sum_{i=1}^{t-1} u_{t} e_{i} \equiv \sum_{i=1}^{t-1} u_{t} u_{i} \equiv 0(\bmod I)
$$

e, similarmente, temos $\bar{g} \bar{u}_{t}=\overline{0}$. Pelo lema (2.16) podemos levantar $\bar{u}_{t}$ a um idempotente não-nulo $e_{t} \in R$ tal que $\bar{e}_{t}=\bar{u}_{t}$ e $g e_{t}=e_{t} g=0$. Agora é fácil ver que $e_{t} e_{i}=e_{t} g e_{i}=0=e_{i} e_{t}, 1 \leqslant i \leqslant t-1$.

Há um exemplo, devido a Zelinsky, de um anel $R$ contendo um ideal nilpotente $N$ tal que uma família $\mathcal{F}$ de idempotentes ortogonais de $R / N$ pode ser levantada a uma família de idempotentes ortogonais de $R$ somente se $\mathcal{F}$ é enumerável; veja [ZE] ou [RO I] (pág. 41). No entanto, como veremos mais tarde, isso não ocorre se $R$ é semiperfeito pois, nesse caso, não existiria uma família infinita de idempotentes ortogonais primitivos - isso será conseqüência da proposição (2.18) e dos teoremas (2.25) e (2.27) a seguir.

Proposição 2.18. Todo anel semiperfeito contém uma família completa de idempotentes ortogonais primitivos.

Prova. Seja $R$ um anel semiperfeito e $J=J(R)$ seu radical. Então $\bar{R}=R / J$ é artiniano semisimples; pelo teorema (1.37) existe uma família completa 
de idempotentes ortogonais primitivos $\left\{\bar{u}_{1}, \ldots, \bar{u}_{t}\right\}$ cuja soma é $\overline{1}$. Pela proposição (2.17), existe uma família de idempotentes ortogonais $\left\{e_{1}, \ldots, e_{t}\right\}$ tais que $\bar{e}_{i}=\bar{u}_{i}, 1 \leqslant i \leqslant t$. Já vimos que esses idempotentes são também primitivos; resta apenas mostrar que $e_{1}+\cdots+e_{t}=1$.

Defina $e=e_{1}+\cdots+e_{t}$. Então $(1-e)^{2}=1-e$, e temos ainda que

$$
1-e \equiv 1-\left(u_{1}+\cdots+u_{t}\right) \equiv 0(\bmod J) \text {. }
$$

Logo $1-e$ é um idempotente em $J$, donde $e=1$ pela proposição (2.14).

Em resumo, se $R$ é semiperfeito toda família completa de idempotentes ortogonais primitivos em $R / J(R)$ pode ser levantada a uma família completa de idempotentes ortogonais primitivos de $R$, e toda família completa de idempotentes ortogonais primitivos em $R$ pode ser projetada sobre uma família completa de idempotentes ortogonais primitivos de $R / J(R)$. Isso conclui o objetivo enunciado à página 15.

O próximo objetivo é descrever a estrutura de anéis semiperfeitos em termos de idempotentes e anéis locais.

Proposição 2.19. Sejam $R$ um anel, e $\in R$ um idempotente e $J=J(R)$. Então $J(e R e)=e R e \cap J=e J e$.

Prova. Seja $M$ um $R$-módulo simples. Se $e M \neq 0$ concluímos que $e M$ é um $e R e$-módulo simples, pois para todo $m \in M$ com $e m \neq 0$ temos

$$
(e R e) e m=e(\operatorname{Rem})=e M,
$$

logo $M$ é simples pela proposição (1.2). Segue da definição de radical que $J(e R e) e M=0$. Se $e M=0$ também temos que $J(e R e) e M=0$, portanto $J(e R e)=J(e R e) e \subseteq J$. Então é claro que $J(e R e) \subseteq e J e$.

Para a inclusão contrária, observe que eRe é um anel com unidade $e$. Se $x \in e J e \subseteq J$, existe $y \in R$ tal que $1=y(1-x)$. Como $x=e x=x e$, temos

$$
e=e y(1-x) e=e y e-e y x e=e y e-e y e x=\operatorname{eye}(e-x),
$$

logo $x$ é quase-regular à esquerda em $e R e$. Segue que $e J e \subseteq J(e R e)$.

Finalmente, é claro que $e R e \cap J=e J e$.

Corolário 2.20. Se e $\in R$ é um idempotente, temos $\frac{e R e+J}{J} \simeq \frac{e R e}{e J e}$.

Prova. Pelo segundo teorema do isomorfismo e por (2.19) podemos escrever

$$
\frac{e R e+J}{J} \simeq \frac{e R e}{e \operatorname{Re} \cap J}=\frac{e R e}{e J e}
$$


Se $R$ é um anel e $e \in R$ é um idempotente, então o anel $e$ Re é chamado de corner ring (veja [LA]). Muitas propriedades de $R$ são herdadas pelo anel $e R e$; por exemplo, ser simples, completamente redutível, semilocal, artiniano, noetheriano, primo, semiprimo, primitivo etc. Vemos, abaixo, um pequeno exemplo disso.

Teorema 2.21. Seja $R$ um anel semiperfeito e $J=J(R)$ seu radical. Se $e \neq 0$ é um idempotente não-nulo de $R$, o anel eRe também é semiperfeito.

Prova. Sejam $L, L^{\prime} \subseteq e R e$ tais que $L, L^{\prime} \supseteq e J e$ e tais que

$$
\frac{L}{e J e} \subsetneq \frac{L^{\prime}}{e J e}
$$

sejam dois ideais à esquerda distintos em eRe/eJe. Considere agora os ideais à esquerda

$$
\frac{R L+J}{J} \quad \text { e } \quad \frac{R L^{\prime}+J}{J}
$$

em $R / J$. Suponha, se possível, que $R L+J=R L^{\prime}+J$; então

$$
e(R L+J) e=e\left(R L^{\prime}+J\right) e
$$

Daí segue que $L+e J e=L^{\prime}+e J e$, uma vez que $e R L e=L$ e eRL'e $=L^{\prime}$. Então $L=L^{\prime}$, contradição; segue que

$$
\frac{R L+J}{J} \subsetneq \frac{R L^{\prime}+J}{J}
$$

em $R / J$, que é artiniano por hipótese. Logo, eRe/eJe também é artiniano.

Seja agora $u \in e R e$ tal que $u^{2}-u \in e J e \subseteq J$. Temos que $\bar{u}=u+J$ é um idempotente do quociente $\bar{R}=R / J$, que $(1-e)$ é um idempotente de $R$ e que $u(1-e)=(1-e) u=0$. Como $R$ é semiperfeito, pelo lema (2.16) temos que $\bar{u}$ pode ser levantado a um idempotente $f \in R$ tal que $f(1-e)=(1-e) f=0$. Daí $f \in e R e, \operatorname{logo} e R e$ é semiperfeito.

O próximo teorema pode ser considerado como um "primeiro teorema de estrutura para anéis semiperfeitos".

Teorema 2.22. Seja $R$ um anel semiperfeito e $J=J(R)$ seu radical. Como grupos abelianos temos $R=S \oplus M$, onde $M$ é um subgrupo do grupo aditivo de $J$ e $S$ é um subanel de $R$ que é soma direta finita de anéis semiperfeitos $R_{1}, \ldots, R_{t}$ tais que $\bar{R}_{i}=R_{i} / J\left(R_{i}\right)$ é um anel simples, $1 \leqslant i \leqslant t$. 
Prova. Como $R$ é semiperfeito, $\bar{R}=R / J$ é artiniano semisimples, donde, pelo teorema de Wedderburn-Artin, temos uma decomposição de $R / J \mathrm{em}$ uma soma direta de ideais bilaterais minimais (simples):

$$
R / J=\bar{R}_{1} \oplus \cdots \oplus \bar{R}_{t}
$$

Pelo teorema (1.40), existe uma família completa de idempotentes primitivos ortogonais centrais $\left\{\bar{e}_{1}, \ldots, \bar{e}_{t}\right\}$ em $\bar{R}$, onde $\bar{e}_{i}$ é o elemento unidade do anel simples $\vec{R}_{i}, 1 \leqslant i \leqslant t$. Como foi mostrado na prova da proposição (2.18), essa família pode ser levantada a uma família completa de idempotentes primitivos ortogonais $\left\{e_{1}, \ldots, e_{t}\right\}$ em $R$. Pelo teorema (2.21), os anéis $e_{i} R e_{i}$ são semiperfeitos e $e_{i} R e_{i} / e_{i} J e_{i} \simeq \bar{R}_{i}$. Obtemos, portanto, uma decomposição de $R$ como soma direta de grupos abelianos:

$$
R=1 R 1=\left(\bigoplus_{1 \leqslant i \leqslant t} e_{i} R e_{i}\right) \oplus\left(\bigoplus_{i \neq j} e_{i} R e_{j}\right)
$$

Façamos

$$
S=\bigoplus_{1 \leqslant i \leqslant t} e_{i} R e_{i} \quad \text { e } \quad M=\bigoplus_{i \neq j} e_{i} R e_{j}
$$

$S$ é uma soma direta (como grupos) dos anéis semiperfeitos $e_{i} R e_{i}$, pois os idempotentes $e_{i}$ são ortogonais. $M$ é um subgrupo do radical $J$. De fato, para todo $r \in R$ temos $i \neq j \Rightarrow \bar{e}_{i} \bar{r} \bar{e}_{j}=\bar{r} \bar{e}_{i} \bar{e}_{j}=\overline{0}$, uma vez que os $\bar{e}_{i}$ são centrais em $\bar{R}$. Observe que $M$ não precisa ser um ideal, já que, por exemplo, $e_{1} R e_{2} \cdot e_{2} R e_{1} \subseteq e_{1} R e_{1}$.

A estrutura dos anéis $e_{i} R e_{i}$ acima será descrita com mais detalhes no teorema (2.29) adiante. Antes disso, precisaremos de mais alguns resultados de caracterização de anéis semiperfeitos em termos de idempotentes locais.

Definição 2.23 (idempotentes locais). Seja $R$ um anel. Um idempotente não-nulo e $\in R$ é chamado local se eRe é um anel local.

Lema 2.24. Seja $R$ um anel e $e \in R$ um idempotente não-nulo. Se e é local, então e é primitivo.

Prova. Se eRe é um anel local, então Re é um ideal à esquerda fortemente indecomponível pelo corolário (2.6). Então Re é indecomponível, logo e é primitivo pelo teorema (1.37).

Em anéis semiperfeitos, idempotentes primitivos e idempotentes locais são a mesma coisa, como veremos no teorema a seguir. 
Teorema 2.25. Seja $R$ um anel semiperfeito e $e \in R$ um idempotente nãonulo. São equivalentes:

(i) e é primitivo.

(ii) Re é um ideal à esquerda indecomponível.

(iii) e é local.

Prova.

(i) $\Leftrightarrow($ ii) Segue do teorema (1.37).

(iii) $\Rightarrow($ i) Lema (2.24).

(i) $\Rightarrow$ (iii) Se $R$ é semiperfeito e $e \in R$ é um idempotente não-nulo, segue do teorema (2.21) que eRe é um anel semiperfeito com unidade $e$, portanto eRe/eJe é um anel artiniano semisimples com unidade é. Pela proposição (2.18), eRe/eJe possui uma família completa de idempotentes não-nulos ortogonais primitivos $\left\{\bar{x}_{1}, \ldots, \bar{x}_{n}\right\}$ tais que $\bar{e}=\bar{x}_{1}+\cdots+\bar{x}_{n}$. Se eRe não é local então eRe/eJe não é um anel de divisão, mas é isomorfo a uma soma direta de anéis de matrizes sobre anéis de divisão, pelo teorema de Wedderburn-Artin. Daí $n \geqslant 2$ e, como eRe é semiperfeito, $\left\{\bar{x}_{1}, \ldots, \bar{x}_{n}\right\}$ pode ser levantada a uma família completa de idempotentes não-nulos ortogonais primitivos $\left\{e_{1}, \ldots, e_{n}\right\} \subseteq e R e$. Como e é a unidade do anel $e R e$, temos ainda que $e=e_{1}+\cdots+e_{n}$, ou seja, e não é primitivo.

Corolário 2.26. Seja $R$ um anel semiperfeito, $J$ seu radical e $\bar{R}=R / J$. Se $e \in R$ é idempotente então Re é indecomponível se e somente se $\bar{R} \bar{e} e ́$ minimal.

Prova. Pelo corolário (2.20), temos

$$
\bar{e} \bar{R} \bar{e}=\frac{e R e+J}{J} \simeq \frac{e R e}{e J e} .
$$

Como $R$ é semiperfeito, eRe também é semiperfeito pelo teorema (2.21), logo $\bar{e} \bar{R} \bar{e}$ é um anel artiniano semisimples. Da proposição (2.19) temos que $J(e R e)=e J e$; usando ainda o resultado do teorema (2.25), conseguimos estabelecer a cadeia de equivalências abaixo:

Re indecomponível $\Longleftrightarrow e$ Re local $\Longleftrightarrow \bar{e} \bar{R} \bar{e}$ local $\Longleftrightarrow \bar{R} \bar{e}$ indecomponível .

Assim, Re é indecomponivel se e somente se $\bar{R} \bar{e}$ é indecomponível. Como $\bar{R}$ é artiniano semisimples então ${ }_{R} \bar{R}$ é um módulo completamente redutível pelo teorema (1.26), donde

$$
\bar{R} \bar{e} \text { indecomponível } \Longleftrightarrow \bar{R} \bar{e} \text { minimal . }
$$

Portanto, Re é indecomponível se e somente se $\bar{R} e \bar{e}$ é minimal. 
Teorema 2.27. Sejam $\mathcal{E}=\left\{e_{1}, \ldots, e_{m}\right\}$ e $\mathcal{F}=\left\{f_{1}, \ldots, f_{n}\right\}$ duas famílias completas de idempotentes locais e ortogonais do anel semilocal $R$. Então $m=n$, e ainda existe um automorfismo interno de $R$ que estabelece uma correspondência bijetora entre $\mathcal{E}$ e $\mathcal{F}$.

Prova.

(1) Primeiramente mostremos que $\bar{R} \bar{e}_{i}$ é um ideal à esquerda minimal de $\bar{R}=R / J(R), 1 \leqslant i \leqslant m$. Ponha $e=e_{i}$ e suponha o caso não-trivial em que $m>1$ (se $m=1, R$ é local e $\bar{R}$ é um anel de divisão). Como

$$
\bar{R}=\bar{R}(\overline{1}-\bar{e})+\bar{R} \bar{e}
$$

e $\bar{R} \bar{e} \neq \overline{0} \neq \bar{R}(\overline{1}-\bar{e})$, pelo lema de Zorn existe um ideal à esquerda maximal $\bar{L}$ em $\bar{R}$ contendo $\bar{R}(\overline{1}-\bar{e})$, donde temos

$$
\bar{R}=\bar{L}+\bar{R} \bar{e} .
$$

Suponha que a soma acima não fosse direta; então, seria $(\bar{L} \cap \bar{R} \bar{e}) \neq \overline{0}$. Como $R$ é semilocal, então $\bar{R}$ é artiniano semisimples, portanto $(\bar{L} \cap \bar{R} \bar{e})^{2} \neq \overline{0}$, uma vez que $\bar{R}$ não conteria ideais à esquerda nilpotentes não-nulos pelo teorema (1.31). Então existiria $x \in R$ tal que $\bar{R} \bar{e} \cdot \bar{x} \bar{e} \neq \overline{0}$ e $\bar{x} \bar{e} \in \bar{L}$. Como eRe é local por hipótese, por (2.19) e (2.20) temos que $\bar{e} \bar{R} \bar{e}$ seria um anel de divisão, portanto o elemento $\bar{e} \bar{x} \bar{e} \neq \overline{0}$ teria um inverso $\bar{y} \in \bar{e} \bar{R} \bar{e}$. Assim, poderíamos escrever

$$
\bar{y} \cdot \bar{e} \bar{x} \vec{e}=\bar{e} \in \bar{L},
$$

donde $\bar{R}=\tilde{L}$, contradizendo o fato de $\bar{L}$ ser maximal. Logo, provamos que

$$
\bar{R}=\bar{L} \oplus \bar{R} \bar{e} \text {. }
$$

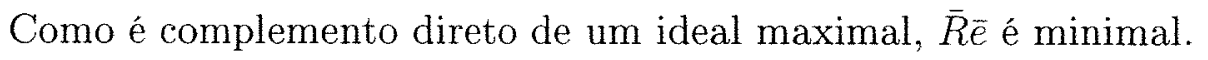

(2) Pelo que foi feito acima, sabemos que

$$
\bar{R} \bar{e}_{1} \oplus \cdots \oplus \bar{R} \bar{e}_{m}=\bar{R} \bar{f}_{1} \oplus \cdots \oplus \bar{R} \bar{f}_{n}
$$

são duas decomposições de ${ }_{\bar{R}} \bar{R}$ como soma direta de $R$-módulos minimais (e, portanto, simples). Pelo teorema de Jordan-Hölder, $m=n$ e existe uma permutação $\sigma \in S_{n}$ tal que

$$
\bar{R} \bar{e}_{1}=\bar{R} \bar{f}_{\sigma(i)},
$$

$1 \leqslant i \leqslant n$. O lema (2.11) assegura a existência de elementos $\bar{u}_{i}, \bar{v}_{i} \in \bar{R}$ tais que $\bar{u}_{i} \bar{v}_{i}=\bar{e}_{i}$ e $\bar{v}_{i} \bar{u}_{i}=\bar{f}_{\sigma(i)}$. Pelo lema (2.12) podemos escrever $u_{i} v_{i}=e_{i}$ e $v_{i} u_{i}=f_{\sigma(i)}$; segue que

$$
\begin{aligned}
v_{i} e_{i} u_{i} & =v_{i}\left(u_{i} v_{i}\right) u_{i}=\left(v_{i} u_{i}\right)\left(v_{i} u_{i}\right)=f_{\sigma(i)}, \\
u_{i} f_{\sigma(i)} v_{i} & =u_{i}\left(v_{i} u_{i}\right) v_{i}=\left(u_{i} v_{i}\right)\left(u_{i} v_{i}\right)=e_{i} .
\end{aligned}
$$


Defina

$$
u=\sum_{i=1}^{n} e_{i} u_{i} f_{\sigma(i)} \quad \text { e } \quad v=\sum_{i=1}^{n} f_{\sigma(i)} v_{i} e_{i}
$$

então, temos

$$
u v=\sum_{i=1}^{n} e_{i}=1 \quad \text { e } \quad v u=\sum_{i=1}^{n} f_{\sigma(i)}=1 .
$$

O elemento $v$ é, pois, invertível em $R$. Como $v e_{i}=f_{\sigma(i)} v_{i} e_{i}=f_{\sigma(i)} v$, segue que $v e_{i} v^{-1}=f_{\sigma(i)}$ e, portanto, $v$ induz um automorfismo interno $x \mapsto v x v^{-1}$ que leva $e_{i}$ em $f_{\sigma(i)}$. Observe que $u$ induz o automorfismo inverso.

O teorema abaixo oferece uma caracterização de anéis semiperfeitos em termos de idempotentes locais.

Teorema 2.28 (B. Müller). Um anel é semiperfeito se e somente se 1 é soma de um número finito de idempotentes locais ortogonais.

\section{Prova.}

$(\Rightarrow)$ Seja $R$ semiperfeito. A proposição (2.18) garante que 1 é a soma de um número finito de idempotentes ortogonais primitivos. Pelo teorema (2.25), idempotentes primitivos são locais.

$(\Leftarrow)$ Seja $\left\{e_{1}, \ldots, e_{n}\right\}$ uma família de idempotentes ortogonais locais tais que $e_{1}+\cdots+e_{n}=1$. Como visto na prova do teorema (2.27), cada $\bar{R} \bar{e}_{i}$ é um ideal à esquerda minimal em $\bar{R}$. Logo

$$
0 \subset \bar{R} \bar{e}_{1} \subset \bar{R} \bar{e}_{1} \oplus \bar{R} \bar{e}_{2} \subset \cdots \subset \bar{R}
$$

é uma série de composição de ${ }_{\bar{R}} \bar{R}$, donde $\bar{R}$ é artiniano. Resta mostrar que idempotentes podem ser levantados.

Seja $\bar{f} \in \widetilde{R}$ um idempotente. Então $\overline{1}-\bar{f}$ é idempotente e, como $\bar{R}$ é artiniano, temos que

$$
\bar{f}=\bar{f}_{1}+\cdots+\bar{f}_{r} \quad \text { e } \quad \overline{1}-\bar{f}=\bar{f}_{r+1}+\cdots+\bar{f}_{t}
$$

são decomposições de $\bar{f}$ e $\overline{1}-\bar{f}$ como somas de idempotentes primitivos ortogonais, correspondentes às decomposições de $\bar{R} \bar{f}$ e $\bar{R}(\overline{1}-\bar{f})$ em somas diretas de ideais à esquerda minimais. $\bar{R}$ é semiperfeito pelo corolário (2.10), donde $\bar{f}_{i}$ é local, $1 \leqslant i \leqslant t$, pelo teorema (2.25). Assim, pelo teorema (2.27), temos que $t=n$ e que existem elementos invertíveis $\bar{u}, \bar{v} \in U(\bar{R})$ definindo um automorfismo interno

$$
\ddot{x} \longmapsto \bar{u} \bar{x} \bar{v}
$$


que leva os $e_{i}$ nos $f_{j}$. Renumerando os índices, se necessário, temos

$$
\bar{u} \bar{e}_{i} \bar{v}=\bar{f}_{i}, \quad 1 \leqslant i \leqslant n,
$$

$\operatorname{logo} \bar{u}\left(\bar{e}_{1}+\cdots+\bar{e}_{r}\right) \bar{v}=\bar{f}$. Defina $e=e_{1}+\cdots+e_{r}$. Se $u$ e $v$ são representantes das classes $\bar{u}$ e $\bar{v}$, temos que $u v=1-w$ para algum $w \in J$. Assim, existe $w^{\prime} \in J$ tal que $\left(1-w^{\prime}\right)(1-w)=1$. Defina

$$
u_{1}=\left(1-w^{\prime}\right) u \quad \text { e } \quad v_{1}=v .
$$

Então $\bar{u}_{1}=\bar{u}$ e $u_{1} v_{1}=1$, donde segue que

$$
v_{1} u_{1} v_{1} u_{1}=v_{1} u_{1} \equiv v u \equiv 1(\bmod J)
$$

e portanto $1-v_{1} u_{1}=\left(1-v_{1} u_{1}\right)^{2} \in J$. Pela proposição (2.14) não há idempotentes não-nulos no radical, $\operatorname{logo} v_{1} u_{1}=1$. A aplicação

$$
x \longmapsto u_{1} x v_{1}
$$

é, portanto, um automorfismo interno de $R$ e, em particular,

$$
u_{1} e v_{1}=u_{1}\left(e_{1}+\cdots+e_{r}\right) v_{1} \equiv f(\bmod J) .
$$

Como $u_{1} e v_{1}$ é um idempotente, levantamos $\tilde{f}$ a um idempotente em $R$.

Nos teoremas (2.29) e (2.30) seguintes, determinamos explicitamente a estrutura de uma classe de anéis semiperfeitos: aqueles cujo quociente pelo seu radical é um anel simples. Esses resultados podem ser vistos como uma generalização do teorema de Wedderburn (1.29).

Teorema 2.29. Seja $R$ um anel semiperfeito tal que $\bar{R}=R / J(R)$ seja um anel simples. Então $R$ é isomorfo ao anel de matrizes $\mathbb{M}_{n}(K)$ sobre o anel local $K=e R e$, onde $e \in R$ é um idempotente local.

Prova. Pelo teorema (2.28), $1 \in R$ pode ser decomposto em uma soma finita de idempotentes ortogonais locais:

$$
1=e_{1}+\cdots+e_{n} .
$$

Então $\overline{1}=\bar{e}_{1}+\cdots+\bar{e}_{n} \in \bar{R}$ é uma decomposição de $\overline{1} \in \bar{R}$ em uma soma de idempotentes ortogonais. Pela proposição (2.15), esses idempotentes são também primitivos; portanto, pelo teorema (2.25) e seu corolário (2.26), $\overline{1}=\bar{e}_{1}+\cdots+\bar{e}_{n} \in \bar{R}$ é uma decomposição de $\overline{1} \in \bar{R}$ como uma soma 
de idempotentes ortogonais locais, cada $e_{i} R$ é indecomponível e cada $\bar{e}_{i} \bar{R}$ é minimal (e, assim, um módulo- $\vec{R}$ simples). Segue que

$$
\bar{R}=\bar{e}_{1} \bar{R} \oplus \cdots \oplus \bar{e}_{n} \bar{R}
$$

é uma decomposição de $\bar{R}$ em uma soma direta de ideais à direita minimais. Como $\bar{R}$ é artiniano simples, possui uma única componente simples, donde $\bar{e}_{i} \bar{R} \simeq \bar{e}_{j} \bar{R}$ para todos $i, j \in\{1, \ldots, n\}$, pela discussão imediatamente após a proposição (1.28). Fixe $e=e_{1}$ e escreva $M=e R$. Então

$$
R_{R} \simeq \underbrace{M \oplus \cdots \oplus M}_{n \text { cópias }},
$$

donde $R \simeq \operatorname{End}\left(R_{R}\right)_{R} \simeq \operatorname{End}\left(M^{n}\right)_{R} \simeq \mathbb{M}_{n}\left(\operatorname{End}(M)_{R}\right)$. Pelo corolário (2.7) temos que $\operatorname{End}(M)_{R} \simeq e R e$ como anéis; se definirmos $K=e R e$, então $K$ é um anel local e temos $R \simeq \mathbb{M}_{n}(K)$.

$\mathrm{Na}$ demonstração acima, trabalhamos com ideais e módulos à direita porque o corolário (2.7) estabelece um isomorfismo de anéis entre $e R e \mathrm{e}$ End $(e R)_{R}$. Adaptando a prova do corolário para o caso de ideais à esquerda, obteríamos um anti-isomorfismo entre eRe e $\operatorname{End}_{R}(R e)$, o que seria menos conveniente.

O teorema abaixo estabelece a recíproca do teorema (2.29).

Teorema 2.30. Um anel $R$ é semiperfeito com $R / J(R)$ simples se e somente se $R$ é isomorfo a um anel de matrizes sobre um anel local.

Prova. A necessidade foi estabelecida no teorema (2.29).

Seja $K$ um anel local e $R=\mathbb{M}_{n}(K)$. Pelo teorema (1.24), temos

$$
\frac{\mathbb{M}_{n}(K)}{J\left(\mathbb{M}_{n}(K)\right)}=\frac{\mathbb{M}_{n}(K)}{\mathbb{M}_{n}(J(K))} \simeq \mathbb{M}_{n}\left(\frac{K}{J(K)}\right) \text {. }
$$

Como $K$ é um anel local concluímos que $K / J(K)$ é um anel de divisão, logo $\mathbb{M}_{n}(K) / J\left(\mathbb{M}_{n}(K)\right)$ é um anel artiniano simples. As matrizes elementares $E_{i i}$ são idempotentes locais (pois $E_{i i} \mathbb{M}_{n}(K) E_{i i} \simeq K$ é local), então a identidade $I=E_{11}+\cdots+E_{n n}$ de $\mathbb{M}_{n}(K)$ é soma de idempotentes locais ortogonais. Logo, $\mathbb{M}_{n}(K)$ é semiperfeito pelo teorema (2.28).

Se $R$ é um anel satisfazendo as condições do teorema (2.30) acima, vimos que $R \simeq \mathbb{M}_{n}(K)$; esse anel de matrizes é único, a menos de isomorfismos. Identifique $R$ com $\mathbb{M}_{n}(K)$ e escreva $R=R E_{11} \oplus \cdots \oplus R E_{n n}$; essa é uma decomposição de Krull-Schmidt do módulo ${ }_{R} R$, donde $n$ está unicamente 
determinado. Todos os somandos diretos dessa decomposição são isomorfos, logo $K$ é isomorfo ao anel de endomorfismos de qualquer um deles. Note que o teorema (2.27) já nos dizia que se $1=e_{1}+\cdots+e_{n}$ é uma decomposição da unidade de $R$ em uma soma de idempotentes ortogonais locais então $n$ é unicamente determinado.

Se $R$ é um anel semiperfeito mas $R / J(R)$ não é simples, podemos ainda dizer que $R / J(R)$ é soma direta de anéis artinianos simples. No entanto, os idempotentes locais centrais dessa decomposição podem não ser levantáveis a idempotentes centrais de $R$. Assim, o resultado do teorema (2.30) não pode ser estendido ao caso geral nos mesmos moldes dos teoremas (1.29) e (1.30). No entanto, essa dificuldade desaparece no caso comutativo, e temos portanto uma caracterização completa dos anéis semiperfeitos comutativos.

Corolário 2.31. Um anel comutativo é semiperfeito se e somente se é soma direta finita de anéis locais comutativos.

Prova. Se $R$ é uma soma direta de anéis locais então $R$ é semilocal, e é claro que os idempotentes de $R / J(R)$ podem ser levantados; logo, $R$ é semiperfeito. Reciprocamente, se $R$ é semiperfeito e comutativo então podemos escrever $1=e_{1}+\cdots+e_{t}$, uma soma de idempotentes locais, pelo teorema (2.28). Portanto $R=R e_{1} \oplus \cdots \oplus R e_{t}$, e cada $R e_{i}=e_{i} R e_{i}$ é um anel local.

\subsection{Anéis Perfeitos}

Veremos agora o conceito de anéis perfeitos. Enquanto anéis semiperfeitos são anéis semilocais cujo radical é "idempotent-lifting", ou seja, anéis $R$ cujos idempotentes de $R / J(R)$ podem ser levantados, anéis perfeitos são anéis semilocais com uma condição um pouco mais forte imposta ao seu radical. Após definir essa condição e caracterizá-la em termos de $R$-módulos, nosso objetivo é o teorema de Bass (2.37), que descreve anéis perfeitos em termos de algumas condições de finitude. Na seção 2.5 veremos, no exemplo (2.64), um anel perfeito unilateral.

Definição 2.32 (T-nilpotência). Um subconjunto $A$ de um anel $R$ é dito $T$-nilpotente à esquerda (respectivamente, à direita) se, para toda seqüência de elementos $\left\{a_{1}, a_{2}, \ldots\right\} \subseteq A$, existe um inteiro $n \geqslant 1$ tal que $a_{1} a_{2} \cdots a_{n}=0$ (respectivamente, $a_{n} \cdots a_{2} a_{1}=0$ ). Dizemos que $A$ é T-nilpotente se o for $\grave{a}$ esquerda e à direita.

Suponha que $I \subseteq R$ é um ideal à esquerda do anel $R$. Se $I$ é $T$-nilpotente à esquerda então é claro que $I$ é um ideal nil, logo todo ideal $T$-nilpotente 
é "idempotent-lifting" (cf. proposição 2.61). Por outro lado, é fácil ver que todo ideal à esquerda nilpotente é $T$-nilpotente.

Definição 2.33 (anéis perfeitos). Um anel $R$ com radical $J=J(R)$ é dito perfeito à esquerda (ou à direita) se $R / J$ é artiniano e $J$ é T-nilpotente à esquerda (ou à direita). Se $R$ é perfeito à esquerda e à direita, dizemos que $R$ é um anel perfeito.

A condição de $T$-nilpotência imposta sobre o radical de um anel perfeito $R$ nos permite concluir fatos sobre $R$-módulos e módulos- $R$. Observe que, no lema a seguir, a condição (ii) se assemelha ao lema de Nakayama, com a condição de finitude de ${ }_{R} M$ substituída pela condição de finitude de $J(R)$.

Lema 2.34. Para todo ideal à esquerda $J$ de um anel $R$ são equivalentes:

(i) $J$ é T-nilpotente à esquerda.

(ii) Se ${ }_{R} M$ é um R-módulo qualquer, $J M=M \Longrightarrow M=0$.

(iii) Se $N_{R}$ é um módulo- $R$ qualquer, $\operatorname{Ann}_{N} J=0 \Longrightarrow N=0$.

$\left(\right.$ Aqui, $\operatorname{Ann}_{N} J$ denota $\{x \in N: x J=0\}$.)

Prova.

$(i) \Rightarrow($ iii $)$ Suponha, por absurdo, que $\operatorname{Ann}_{N} J=0 \neq N$. Tome um elemento não-nulo $x \in N$; então $x \notin \operatorname{Ann}_{N} J$, donde existe $a_{1} \in J$ tal que $x a_{1} \neq 0$. Então $x a_{1} \notin \operatorname{Ann}_{N} J$, donde existe $a_{2} \in J$ tal que $x a_{1} a_{2} \neq 0$. Continuando dessa maneira, temos uma seqüência $\left\{a_{1}, a_{2}, \ldots\right\} \subseteq J$ tal que $a_{1} a_{2} \cdots a_{n} \neq 0$ para todo inteiro positivo $n$, portanto $J$ não é $T$-nilpotente à esquerda.

(iii) $\Rightarrow$ (ii) Suponha que ${ }_{R} M \neq 0$ e defina $A=\operatorname{Ann}_{R} M$; então $A$ é um ideal bilateral próprio de $R$. Defina $N=R / A$; considerando $N=R / A$ como um módulo- $R$, temos que $\operatorname{Ann}_{N} J$ é dado por $B / A$, onde $B \subseteq R$ é um ideal bilateral dado por

$$
B=\{b \in R: b J \subseteq A\} .
$$

Como $N \neq 0$, por (iii) temos que $\operatorname{Ann}_{N} J=B / A \neq 0$, ou seja, $A$ é um ideal próprio de $B$. Portanto, $B M \neq 0$. Por outro lado, $B J \subseteq A$ implica que $B J M \subseteq A M=0, \log 0, J M \neq M$.

(ii $) \Rightarrow\left(\right.$ i) Considere uma seqüência qualquer $\left\{a_{1}, a_{2}, \ldots\right\} \subseteq J$, e considere o $R$-módulo livre $F=\bigoplus_{i=0}^{\infty} R e_{i}$ com base $\left\{e_{i}: i \geqslant 0\right\}$. Defina $S$ como o $R$-submódulo de $F$ gerado por

$$
s_{0}=e_{0}-a_{1} e_{1}, \quad s_{1}=e_{1}-a_{2} e_{2}, \quad s_{2}=e_{2}-a_{3} e_{3}, \quad \text { etc }
$$


Defina $M=F / S=\bar{F}$. Em $M$ temos, portanto, as relações

$$
\bar{e}_{0}=a_{1} \bar{e}_{1} \quad \bar{e}_{1}=a_{2} \bar{e}_{2} \quad \bar{e}_{2}=a_{3} \bar{e}_{3} \quad \ldots
$$

É claro que $J M \subseteq M$; para a inclusão contrária, tome $m \in M$ e observe que

$$
m=\sum_{i=0}^{k} r_{i} \bar{e}_{i}=\sum_{i=0}^{k} r_{i} a_{i+1} \bar{e}_{i+1} \in J M
$$

para algum inteiro positivo $k$. Então $M=J M$ e, pela hipótese (ii), $M=0$, donde segue que $S=F$. Em particular, expressando $e_{0}$ na base $\left\{s_{j}: j \geqslant 0\right\}$ obtemos

$$
e_{0}=b_{1}\left(e_{0}-a_{1} e_{1}\right)+\cdots+b_{n}\left(e_{n-1}-a_{n} e_{n}\right)
$$

para certos $b_{i} \in R$ e algum inteiro positivo $n$. Comparando os coeficientes $\operatorname{dos} e_{i}$ obtemos $b_{1}=1, b_{2}=b_{1} a_{1}=a_{1}, b_{3}=b_{2} a_{2}=a_{1} a_{2}$ etc. Por indução segue facilmente que $b_{n}=b_{n-1} a_{n-1}=a_{1} a_{2} \cdots a_{n-1}$, e finalmente

$$
0=b_{n} a_{n}=a_{1} a_{2} \cdots a_{n},
$$

provando que $J$ é $T$-nilpotente à esquerda.

Precisaremos de um último resultado auxiliar antes do teorema de Bass.

Definição 2.35 (anéis semiprimos). Um anel $R$ é dito semiprimo se, para todo ideal $I \subseteq R$, temos $I^{2}=0 \Longrightarrow I=0$.

Proposição 2.36. São equivalentes as afirmações abaixo:

(i) $R$ é semiprimo.

(ii) R não tem ideais nilpotentes não-nulos.

(iii) $R$ não tem ideais à esquerda nilpotentes não-nulos.

Prova. As implicações $(i i i) \Rightarrow(i i)$ e $(i i) \Rightarrow(i)$ são triviais. Para estabelecer $(i) \Rightarrow$ (iii), considere um anel semiprimo $R$ e um ideal à esquerda nilpotente $I \subseteq R$. Escolha o menor inteiro positivo $n$ tal que $I^{n}=0$. Se $n>1$ então $2 n-2 \geqslant n$, donde

$$
\left(I^{n-1}\right)^{2}=I^{2 n-2} \subseteq I^{n}=0 \Longrightarrow I^{n-1}=0,
$$

em contradição com a escolha de $n$. Assim, $n=1$ e $I=0$.

O teorema de Bass (vide $[\mathrm{BA}]$ ) caracteriza anéis perfeitos em termos de condições de finitude sobre módulos, ideais e idempotentes. 
Teorema 2.37 (Bass). Para qualquer anel $R$, são equivalentes:

(i) $R$ é perfeito à esquerda.

(ii) R satisfaz a CCD em ideais à direita principais.

(iii) Todo módulo $N_{R}$ satisfaz a CCD em submódulos cíclicos.

(iv) $R$ não contém uma família ortogonal infinita de idempotentes nãonulos, e todo módulo não-nulo $N_{R}$ contém um submódulo simples.

Prova. $(i) \Rightarrow($ ii $)$ é uma implicação cuja prova será adiada para a seção 2.4. Aqui, provaremos apenas $(i i) \Rightarrow(i i i) \Rightarrow(i v) \Rightarrow(i)$.

(ii) $\Rightarrow($ iii $)$ Dados $x, y \in N_{R}$, é claro que $x R \supseteq y R \Rightarrow y \in x R \Rightarrow y=x a_{1}$ para algum $a_{1} \in R$. Sendo assim, toda cadeia descendente de submódulos cíclicos em $N_{R}$ pode ser expressa na forma

$$
x R \supseteq x a_{1} R \supseteq x a_{1} a_{2} R \supseteq \cdots
$$

para alguns elementos $a_{1}, a_{2}, \ldots$ de $R$. Por (ii), a cadeia

$$
R \supseteq a_{1} R \supseteq a_{1} a_{2} R \supseteq \cdots
$$

estabiliza; logo, a cadeia (2.2) também estabiliza.

(iii) $\Rightarrow($ iv $)$ Por (iii), existe um elemento $x \in N_{R} \neq 0$ tal que $x R$ é minimal como submódulo cíclico não-nulo. Claramente, $x R$ é um submódulo simples de $N_{R}$.

Suponha, por absurdo, que $R$ contém uma família ortogonal infinita de idempotentes não-nulos $\left\{e_{1}, e_{2}, \ldots\right\}$. Então cada $1-e_{1}-e_{2}-\cdots-e_{n}$ é um idempotente, e temos

$$
\left(1-e_{1}\right) R \supseteq\left(1-e_{1}-e_{2}\right) R \supseteq\left(1-e_{1}-e_{2}-e_{3}\right) R \supseteq \cdots,
$$

uma vez que $\left(1-e_{1}-\cdots-e_{n}\right)\left(1-e_{n+1}\right)=1-e_{1}-\cdots-e_{n}-e_{n+1}$ para todo inteiro positivo $n$. Teremos a contradição desejada se pudermos provar que as inclusões em (2.3) são todas estritas. De fato, se

$$
\left(1-e_{1}-\cdots-e_{n}\right) R=\left(1-e_{1}-\cdots-e_{n+1}\right) R
$$

para algum inteiro positivo $n$, temos

$$
1-e_{1}-\cdots-e_{n}=\left(1-e_{1}-\cdots-e_{n+1}\right) r
$$


para algum $r \in R$. Multiplicando à esquerda por $e_{n+1}$ obteríamos

$$
e_{n+1}=e_{n+1} r-e_{n+1}^{2} r=0
$$

o que não é possível.

$(i v) \Rightarrow(i)$ Seja $N_{R} \neq 0$ um módulo- $R$ não-nulo qualquer. Por (iv), existe um submódulo simples $N_{0} \subseteq N$. Como o radical $J=J(R)$ é a interseção de todos os anuladores de módulos- $R$ simples, então

$$
\operatorname{Ann}_{N} J=\{x \in N: x J=0\} \supseteq N_{0}
$$

de modo que $\operatorname{Ann}_{N} J \neq 0$. Temos, portanto, que $\operatorname{Ann}_{N} J=0 \Rightarrow N=0$, donde $J$ é $T$-nilpotente à esquerda pelo lema (2.34). Resta apenas mostrar que o anel $S=\bar{R}=R / J$ é artiniano.

Como o radical $J(S)$ é nulo, $S$ não tem ideais nilpotentes não-nulos pelo teorema (1.24). Pela proposição (2.36), $S$ é um anel semiprimo. Portanto, pelo lema de Brauer (1.39), todo ideal à direita minimal $\bar{D} \subseteq \bar{R}$ pode ser escrito na forma $\bar{D}=\bar{e} S$ para algum idempotente primitivo $\bar{e} \in S$. Assim, todo ideal à direita minimal de $S$ é um somando direto, logo todo ideal à direita minimal de $S$ é simples como módulo- $R$. Em resumo, sobre o módulo $S_{R}$ podemos afirmar:

(a) todo submódulo não-nulo de $S_{R}$ contém um submódulo simples;

(b) todo submódulo simples de $S_{R}$ é um somando direto.

Suponha, por absurdo, que $S_{R}$ não seja artiniano à direita. Por $(a)$ e $(b)$ acima, existiriam decomposições

$$
S=\bar{A}_{1} \oplus \bar{B}_{1}, \quad \bar{B}_{1}=\bar{A}_{2} \oplus \bar{B}_{2}, \quad \bar{B}_{2}=\bar{A}_{3} \oplus \bar{B}_{3} \quad \text { etc. },
$$

sendo os $\bar{A}_{i}$ ideais à direita minimais de $S$. Continuando essa construção, obteríamos uma família ortogonal infinita de idempotentes primitivos nãonulos $\left\{\bar{e}_{i}: i \geqslant 0\right\}$ tal que $\bar{A}_{i}=\bar{e}_{i} \bar{R}_{\bar{e}}$ para todo $i$. Como $J$ é $T$-nilpotente à esquerda então $J$ é nil, logo os idempotentes $\left\{\bar{e}_{i}: i \geqslant 0\right\}$ poderiam ser levantados a uma família infinita $\left\{e_{i}: i \geqslant 0\right\}$ de idempotentes não-nulos ortogonais primitivos de $R$ pelas proposições (2.16) e (2.17). Isso contraria a hipótese (iv), logo $S$ é artiniano à direita.

Nesse ponto relembramos que o conceito de anel completamente redutível é simétrico; assim, como $J(S)=0$ então $S$ é artiniano à esquerda se e somente se $S$ é artiniano à direita. Como $S_{R}$ é semisimples então $S$ é artiniano, como queríamos demonstrar. 


\subsection{Caracterizações Homológicas}

O objetivo desta seção é completar a demonstração do teorema de Bass (2.37), enunciado à página 31. A demonstração da implicação $(i) \Rightarrow(i i)$ será realizada utilizando alguns conceitos de Álgebra Homológica, como módulos planos, módulos projetivos e sequiências exatas. Ao longo da exposição serão destacadas as caracterizações homológicas de anéis perfeitos e semiperfeitos descobertas por Bass.

Em toda esta seção, $R$ denota um anel com unidade.

Definição 2.38 (submódulos supérfluos). Seja $M$ um R-módulo. Um $R$-submódulo $S \subseteq M$ é dito supérfluo, o que será denotado por $S \subseteq_{s} M$, se, para todo $R$-submódulo $N \subseteq M$, valer a implicação

$$
S+N=M \Longrightarrow N=M .
$$

De forma análoga, dizemos que um ideal à esquerda $L$ de um anel $R$ é supérfluo se ${ }_{R} L$ é um $R$-submódulo supérfluo em ${ }_{R} R$. Observe, por exemplo, que um somando direto não-nulo de um $R$-módulo $M$ nunca é supérfluo.

Exemplo 2.39. Um ideal à esquerda $L$ de um anel $R$ é supérfluo se e somente se $L \subseteq J(R)$.

Com efeito, como $J(R)$ é o conjunto de todos os não-geradores de $R$, se $L \subseteq J(R)$ então $L$ é um conjunto não-gerador, donde $L$ é supérfluo.

Reciprocamente, sejam $L \subseteq R$ um ideal à esquerda supérfluo e $r \in L$. Então $r$ está contido em qualquer ideal à esquerda maximal $M \subseteq R$, pois do contrário $M \cup\{r\}$ seria gerador de $R$, pela maximalidade de $M$. Assim, $r \in J(R)$ e, portanto, $L \subseteq J(R)$.

A seguir desenvolveremos a idéia de radical de um módulo, análoga à de radical de um anel.

Definição 2.40 (radical de um módulo). $O$ radical de um $R$-módulo $M$ é o submódulo $\mathrm{rad} M$ definido como a interseção de todos os R-submódulos maximais de $M$. Se $M$ não tem submódulos maximais, define-se $\operatorname{rad} M=M$.

Proposição 2.41. Seja $M$ um R-módulo e $J=J(R)$. Então

(i) $\operatorname{rad} M$ é a soma de todos os submódulos supérfluos de $M$.

(ii) $J M \subseteq \operatorname{rad} M$, com igualdade se $R$ é semilocal. 


\section{Prova.}

(i) Defina $T=\sum\left\{S: S \subseteq_{s} M\right\}$. Se $N$ é um $R$-submódulo maximal qualquer de $M$, então é claro que $N$ contém todos os submódulos supérfluos de $M$. Logo todo submódulo supérfluo de $M$ está contido na interseção de todos os submódulos maximais de $M$, ou seja, $T \subseteq \operatorname{rad} M$.

Para a inclusão contrária, basta mostrar que $R m \subseteq_{s} M$ para qualquer $m \in \operatorname{rad} M$, pois nesse caso teríamos

$$
\operatorname{rad} M \subseteq R \cdot \operatorname{rad} M=\sum_{m \in \operatorname{rad} M} R m \subseteq T
$$

Considere então um $R$-submódulo qualquer $N \subseteq M$ tal que $N+R m=M$. Suponha inicialmente que $m \notin N$. Então $M / N$ é um $R$-submódulo cíclico não-nulo (gerado pela classe $m+N$ ), donde $M / N$ possui um $R$-submódulo maximal $N^{\prime} / N$. Então $N^{\prime}$ é um $R$-submódulo maximal próprio de $M$ que contém $N$, donde $m \notin N^{\prime}$, contradizendo a hipótese de que $m \in \operatorname{rad} M$. Daí $m \in N$ e $M=N+R m=N$, ou seja, $R m$ é mesmo supérfluo em $M$.

(ii) Para todo $R$-submódulo maximal $N \subset M$, o quociente $M / N$ é simples e, portanto, anulado à esquerda por $J$ (por definição). Disso segue que $J M \subseteq N$.

Suponha que $R$ é semilocal, ou seja, que $R / J$ é artiniano semisimples. Como $M / J M$ é um $R$-módulo e $J \subseteq \operatorname{Ann}_{R}(M / J M)$, da proposição (1.7) segue que $M / J M$ é um $(R / J)$-módulo com o mesmo reticulado de submódulos de ${ }_{R}(M / J M)$. Do teorema $(1.26)(i)$ temos que $M / J M$ é completamente redutível, ou seja, todo $R$-submódulo não-nulo de $M / J M$ é um somando direto. Do item $(i)$ provado acima segue que $\operatorname{rad}(M / J M)=0$. Finalmente, é claro que $\operatorname{rad}(M / J M)=(\operatorname{rad} M) / J M, \operatorname{logo}$ temos $J M=\operatorname{rad} M$.

Proposição 2.42. Sejam $M, M^{\prime} R$-módulos e $J=J(R)$.

(i) $M^{\prime} \subseteq M \Longrightarrow \operatorname{rad} M^{\prime} \subseteq \operatorname{rad} M$.

(ii) $\operatorname{rad}\left(\bigoplus_{i \in I} M_{i}\right)=\bigoplus_{i \in I} \operatorname{rad} M_{i}$

(iii) Se ${ }_{R} F$ é livre sobre $R$, então $\operatorname{rad} F=J F$.

\section{Prova.}

(i) Seja $M^{\prime} \subseteq M$. Primeiro vejamos que, se $S \subseteq_{s} M^{\prime}$ é um $R$-submódulo, então $S \subseteq \subseteq_{s} M$. De fato, se $S+N=M$ para algum $R$-submódulo $N \subseteq M$ então $S+\left(N \cap M^{\prime}\right)=M^{\prime}$, $\log o N \cap M^{\prime}=M^{\prime}$, ou seja, $S \subseteq M^{\prime} \subseteq N$. Então $M=S+N=N$, donde $S \subseteq_{s} M$ como queríamos. $\operatorname{rad} M^{\prime}$ é a soma de todos os $R$-submódulos supérfluos de $M^{\prime}$ por (2.41), portanto $\operatorname{rad} M^{\prime} \subseteq \operatorname{rad} M$. 
(ii) Provaremos esse fato para o caso particular $I=\{1,2\}$; o argumento no caso geral é similar, apenas com notação mais complicada.

Defina $T=\operatorname{rad}\left(M_{1} \oplus M_{2}\right)$; do item $(i)$, obtemos $T \supseteq \operatorname{rad} M_{1} \oplus \operatorname{rad} M_{2}$. Considere agora $m=\left(m_{1}, m_{2}\right) \in T$. Se $N \subset M_{1}$ é um $R$-submódulo maximal qualquer de $M_{1}$, então é claro que $N \oplus M_{2}$ é um $R$-submódulo maximal de $M_{1} \oplus M_{2}, \operatorname{logo} m \in N \oplus M_{2}$; segue que $m_{1} \in N$. Então $m_{1}$ pertence a todos os $R$-submódulos maximais de $M_{1}$; por definição, temos que $m_{1} \in \operatorname{rad} M_{1}$. Analogamente, temos $m_{2} \in \operatorname{rad} M_{2}$. Isso mostra que $m \in \operatorname{rad} M_{1} \oplus \operatorname{rad} M_{2}$, ou seja, $\operatorname{rad}\left(M_{1} \oplus M_{2}\right)=\operatorname{rad} M_{1} \oplus \operatorname{rad} M_{2}$.

(iii) Seja $\left\{e_{i}\right\}_{i \in I}$ uma base de ${ }_{R} F$ sobre $R$. Como $R=R e_{i}$ são isomorfos (como $R$-módulos) pelo isomorfismo $r \mapsto r e_{i}$, é fácil ver que $\operatorname{rad}\left(R e_{i}\right)=J e_{i}$. Pelo item (ii), podemos escrever

$$
\operatorname{rad} F=\operatorname{rad}\left(\bigoplus_{i \in I} R e_{i}\right)=\bigoplus_{i \in I} \operatorname{rad}\left(R e_{i}\right)=\bigoplus_{i \in I} J e_{i}=J \bigoplus_{i \in I} R e_{i}=J F
$$

Proposição 2.43. Seja $P$ um $R$-módulo projetivo não-nulo. $S e J=J(R)$ então $\operatorname{rad} P=J P \subsetneq P$.

Prova. Todo módulo projetivo é somando direto de algum módulo livre; assim, existe um $R$-módulo projetivo $Q$ tal que $P \oplus Q$ é o $R$-módulo livre $F=\bigoplus_{i \in I} R e_{i}$. Da proposição (2.42), temos

$$
\operatorname{rad} P \oplus \operatorname{rad} Q=\operatorname{rad} F=J F=J P \oplus J Q
$$

portanto, $J P=\operatorname{rad} P$.

Falta mostrar que $J P \neq P$. Suponha, do contrário, que $J P=P$ e defina

$$
p=\sum_{i=1}^{n} r_{i} e_{i} \in P
$$

(usando inteiros positivos como elementos do conjunto de índices $I$ para simplificar a notação). Defina ainda $\pi$ a projeção de $F=P \oplus Q$ sobre $P$. Como $P=J P \subseteq J F$, podemos escrever

$$
\pi\left(e_{i}\right)=\sum_{j=1}^{m} a_{i j} e_{j}
$$

onde $a_{i j} \in J$ e $m \geqslant n$ é algum inteiro positivo. Então

$$
p=\pi(p)=\sum_{i=1}^{n} r_{i} \pi\left(e_{i}\right)=\sum_{i=1}^{n} r_{i} \sum_{j=1}^{n} a_{i j} e_{j}=\sum_{j=1}^{m}\left(\sum_{i=1}^{n} r_{i} a_{i j}\right) e_{j} .
$$


Comparando com $p=\sum_{j=1}^{n} r_{j} e_{j}$, obtemos um sistema linear homogêneo de $n$ equações:

$$
\sum_{i=1}^{n} r_{i}\left(\delta_{i j}-a_{i j}\right)=0, \quad j=1, \ldots, n .
$$

A matriz dos coeficientes desse sistema pertence a

$$
I_{n}+\mathbb{M}_{n}(J)=I_{n}+J\left(\mathbb{M}_{n}(R)\right) \subseteq U\left(\mathbb{M}_{n}(R)\right)
$$

portanto o sistema só admite a solução trivial $r_{1}=\cdots=r_{n}$. Então $p=0$, contradizendo o fato de que $P$ não é um módulo nulo.

A seguir, veremos o conceito de cobertura projetiva.

Definição 2.44 (cobertura projetiva). Uma cobertura projetiva de um $R$-módulo $M$ é um módulo projetivo ${ }_{R} P$ com um epimorfismo $\theta: P \rightarrow M$ de núcleo supérfluo, ou seja, tal que $\operatorname{ker} \theta \subseteq s$.

Um módulo pode não ter cobertura projetiva, como será visto no exemplo (2.68). O lema abaixo nos dá uma condição necessária para que ker $\theta \subseteq_{s} P$.

Lema 2.45. Sejam $M$ um $R$-módulo e $\theta: P \rightarrow M$ uma cobertura projetiva de $M$. Então, para todo $R$-submódulo $P^{\prime} \subseteq P, \theta\left(P^{\prime}\right)=M \Longrightarrow P^{\prime}=P$.

Prova. Se $\theta\left(P^{\prime}\right)=M$, para todo $p \in P$ temos $\theta(p)=\theta\left(p^{\prime}\right)$ para algum $p^{\prime} \in P^{\prime}$. Então $p-p^{\prime} \in \operatorname{ker} \theta$, donde $P=P^{\prime}+\operatorname{ker} \theta$. Como $\operatorname{ker} \theta$ é supérfluo em $P$, temos $P^{\prime}=P$.

Lema 2.46. Sejam $M$ um $R$-módulo e $J=J(R)$.

(i) Se $M$ é finitamente gerado ou $J$ é T-nilpotente à esquerda, então $J M \subseteq_{s} M$.

(ii) Se um $R$-submódulo $S \subseteq M$ é supérfluo em $M$, então todo $R$-submódulo de $S$ é supérfluo em $M$.

Prova. (i) Se $M$ é um $R$-módulo finitamente gerado, o resultado segue do lema de Nakayama; se $J$ é $T$-nilpotente à esquerda, o resultado segue do lema (2.34). O item (ii) é evidente.

Proposição 2.47 (unicidade da cobertura projetiva). Sejam $M u m R$ módulo e $\theta: P \rightarrow M$ uma cobertura projetiva de $M$. Se $\theta^{\prime}: P^{\prime} \rightarrow M$ é outra cobertura projetiva, existe um isomorfismo $\alpha: P^{\prime} \rightarrow P$ tal que $\theta \circ \alpha=\theta^{\prime}$. 
Prova. Como $P^{\prime}$ é projetivo, existe um homomorfismo $\alpha: P^{\prime} \rightarrow P$ tal que $\theta \circ \alpha=\theta^{\prime}$, ou seja, tal que o diagrama abaixo comuta:

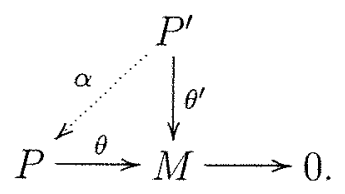

Então $\alpha\left(P^{\prime}\right)$ é um submódulo de $P$ tal que $\theta\left(\alpha\left(P^{\prime}\right)\right)=M$; pelo lema (2.45) temos $\alpha\left(P^{\prime}\right)=P$, ou seja, $\alpha$ é epimorfismo.

Como $P$ é projetivo, pela proposição (1.44) a seqüência exata

$$
0 \longrightarrow \operatorname{ker} \alpha \longrightarrow P^{\prime} \stackrel{\alpha}{\longrightarrow} P \longrightarrow 0
$$

cinde, ou seja, ker $\alpha$ é um somando direto de $P^{\prime}$. No entanto, observe que $\operatorname{ker} \alpha \subseteq \operatorname{ker} \theta^{\prime}, \operatorname{logo}$ ker $\alpha \subseteq_{s} P^{\prime}$ pelo lema (2.46). Por ser um somando direto supérfluo de $P^{\prime}$, ker $\alpha=0$ e, portanto, $\alpha$ é um isomorfismo.

Nosso próximo objetivo é descrever os anéis perfeitos e semiperfeitos em termos de coberturas projetivas de seus módulos. Será provado que os anéis semiperfeitos são anéis cujos módulos finitamente gerados têm cobertura projetiva, enquanto anéis perfeitos são anéis cujos módulos quaisquer têm cobertura projetiva. Note que, nos teoremas abaixo, a condição de finitude ( $T$-nilpotência) do radical de um anel perfeito $R$ elimina a hipótese de que $R$-módulos, sobre os quais se afirma algo, sejam finitamente gerados. Isso, em geral, é uma constante nos resultados sobre anéis perfeitos e semiperfeitos.

Teorema 2.48. Se $R$ é um anel semiperfeito, todo módulo finitamente gerado sobre $R$ tem cobertura projetiva. Se $R$ é perfeito à esquerda, todo $R$-módulo tem cobertura projetiva.

Prova. Seja $J=J(R)$ e $\bar{R}=R / J$. Suponha, primeiramente, que $R$ é semiperfeito. Pelo teorema (2.28), $R$ tem uma família completa $\left\{e_{1}, \ldots, e_{n}\right\}$ de idempotentes locais ortogonais. Portanto

$$
\bar{R}=\bar{R} \bar{e}_{1} \oplus \cdots \oplus \bar{R} \bar{e}_{n}
$$

e todo $\bar{R}$-módulo simples é isomorfo a um dos $\bar{R} \bar{e}_{i}$. Seja $M$ um $R$-módulo qualquer. Como foi visto na demonstração da proposição (2.41), M/JM é um $\bar{R}$-módulo; como $\bar{R}$ é artiniano semisimples, $M / J M$ é completamente redutível e pode ser escrito na forma

$$
\frac{M}{J M} \simeq \bigoplus_{\alpha \in I} \bar{R} \bar{e}_{\alpha}
$$


sendo $I$ um conjunto de índices e $e_{\alpha} \in\left\{e_{1}, \ldots, e_{n}\right\}$ para todo $\alpha \in I$.

Considere agora o $R$-módulo

$$
P=\bigoplus_{\alpha \in I} R e_{\alpha} .
$$

Todo $R e_{\alpha}$ é somando direto do módulo livre ${ }_{R} R$ e $P$ é uma soma direta dos $R e_{\alpha}$; das proposições (1.44) e (1.46) segue que $P$ é projetivo. Como $\bar{R} \bar{e}_{\alpha} \simeq R e_{\alpha} / J e_{\alpha}$, temos o seguinte diagrama comutativo, com linha inferior exata:

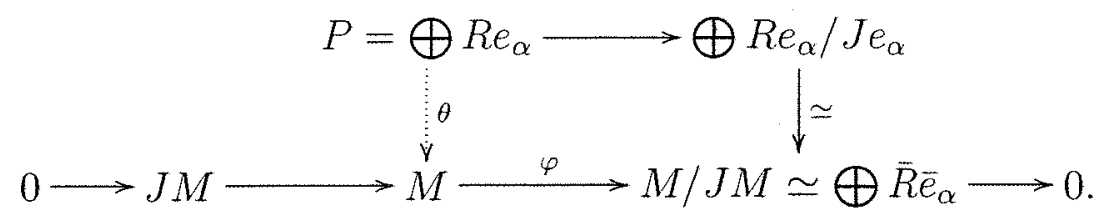

Pelo diagrama, podemos afirmar que:

(a) $\theta(P)+J M=M$; de fato, para todo $m \in M$ existe $p \in P$ tal que $m+J M=\theta(p)+J M$; então $m=\theta(p)+\sum j_{i} m_{i} \in \theta(P)+J M$.

(b) $\operatorname{ker} \theta \subseteq \oplus J e_{\alpha}=J P$; pode ser verificado diretamente no diagrama.

${ }_{R} M$ é finitamente gerado, $\log$ o $I$ é finito e $P$ é finitamente gerado. Pelo lema de Nakayama, a afirmação (a) implica que $\theta(P)=M$. Pelo lema (2.46), $J P \subseteq_{s} P$; logo, a afirmação (b) e o lema (2.46) implicam que $\operatorname{ker} \theta \subseteq_{s} P$. Assim, $\theta: P \rightarrow M$ é uma cobertura projetiva de $M$.

Suponha, agora, que ${ }_{R} M$ é um módulo arbitrário e que $R$ é perfeito à esquerda. $R$ também é semiperfeito, pela proposição (2.61), portanto o diagrama acima e as afirmações (a) e (b) continuam válidas. $J$ é $T$-nilpotente à esquerda, donde, pelo lema (2.46), $J N \subseteq_{s} N$ para todo $R$-módulo $N$. Da afirmação (a) acima segue que $\theta(P)=M$. Do lema (2.46) temos $J P \subseteq_{s} P$, portanto a afirmação (b) implica que $\operatorname{ker} \theta \subseteq_{s} P$. Assim, $\theta: P \rightarrow M$ é uma cobertura projetiva de $M$.

O próximo passo é estabelecer a recíproca do teorema acima e, assim, concluir a caracterização de anéis semiperfeitos e perfeitos à esquerda em termos de coberturas projetivas.

\section{Observação}

Se $I$ é um ideal do anel $R$ e $S=R / I$, observe que se $M$ é um $S$-módulo então $M$ é também um $R$-módulo, com ação definida por $r m=(r+I) m$ para todos $r \in R, m \in M$. 
Lema 2.49. Seja I um ideal do anel $R$, e escreva $S=R / I$. Se ${ }_{R} M$ tem uma cobertura projetiva sobre $R$, então ${ }_{S} M$ tem uma cobertura projetiva sobre $S$.

Prova. Seja $\theta: P \rightarrow{ }_{R} M$ uma cobertura projetiva de ${ }_{R} M$, e considere a aplicação $\bar{\theta}: \bar{P}=P / I P \rightarrow{ }_{S} M$ dada por $\tilde{\theta}(\bar{p})=\theta(p)$ para todo $\bar{p} \in \bar{P}$. É fácil ver que $\bar{\theta}$ está bem definida, pois para todos $\bar{p}, \bar{q} \in \bar{P}$ temos

$$
\bar{p}=\bar{q} \Longrightarrow p-q \in I P \Longrightarrow \theta(p-q) \in I M=0 \Longrightarrow \bar{\theta}(\bar{p})=\bar{\theta}(\bar{q}) .
$$

É claro que $\operatorname{ker} \bar{\theta}=\operatorname{ker} \theta / I P$. Suponha agora que

$$
\frac{N}{I P}+\frac{\operatorname{ker} \theta}{I P}=\frac{P}{I P}
$$

para algum $R$-submódulo $N \subseteq P$. Isso implica que $N+\operatorname{ker} \theta=P$, portanto $N=P$, uma vez que $\operatorname{ker} \theta$ é supérfluo em $P$. Então ker $\bar{\theta}$ é supérfluo em $P / I P$. Finalmente, $P / I P$ é projetivo como $S$-módulo pelo lema (1.47), logo $\bar{\theta}: P / I P \rightarrow{ }_{S} M$ é uma cobertura projetiva de ${ }_{S} M$.

Lema 2.50. Sejam $R$ um anel tal que $J(R)=0$ e $M$ um $R$-módulo que admite uma cobertura projetiva sobre $R$. Então, $M$ é um módulo projetivo.

Prova. Seja $\theta: P \rightarrow M$ uma cobertura projetiva de $M$. Então ker $\theta$ é supérfluo em $P$. Como $P$ é projetivo e $J=0$, da proposição (2.43) segue que $\operatorname{rad} P=J P=0$. Mas $\operatorname{rad} P$ é soma de todos os submódulos supérfluos de $P$ pela proposição (2.41), logo $\operatorname{ker} \theta=0$. Portanto, $\theta$ é um isomorfismo e $M$ é projetivo.

Teorema 2.51 (caracterização homológica de anéis semiperfeitos). São equivalentes as afirmaçôes:

(i) R é semiperfeito.

(ii) Todo R-módulo finitamente gerado tem uma cobertura projetiva.

(iii) Todo R-módulo cíclico tem uma cobertura projetiva.

Prova. A implicação (i) $\Rightarrow$ (ii) é o teorema (2.48), e a implicação (ii) $\Rightarrow$ (iii) é trivial; portanto, resta apenas provar que (iii) $\Rightarrow(i)$.

Primeiro vejamos que os idempotentes de $S=\vec{R}=R / J(R)$ podem ser levantados. Se $u \in S$ é idempotente então ${ }_{R} S=S u \oplus S v$, sendo $v=\overline{1}-u$ idempotente. Por hipótese, existem coberturas projetivas

$$
\alpha:{ }_{R} P \longrightarrow S u \quad \text { e } \quad \beta:{ }_{R} Q \longrightarrow S v .
$$


Não é difícil ver que

$$
\alpha \oplus \beta: P \oplus Q \longrightarrow S u \oplus S v={ }_{R} S
$$

é uma cobertura projetiva de ${ }_{R} S$. Por outro lado,

$$
\pi:{ }_{R} R \longrightarrow{ }_{R} S
$$

também é uma cobertura projetiva de ${ }_{R} S$. De fato, sabemos que ${ }_{R} R$ é projetivo e que $J(R)=\operatorname{ker} \pi$. Ainda, $J(R)$ é supérfluo em ${ }_{R} R$ pelo lema (2.46). Pela unicidade da cobertura projetiva (2.47), existe um isomorfismo $\varphi: P \oplus Q \rightarrow R$ tal que $\pi \circ \varphi=\alpha \oplus \beta$. Portanto, podemos identificar $P$ e $Q$ com ideais à esquerda de $R$ tais que $\pi(P)=S u, \pi(Q)=S v$. Seja $1=e+f$ a decomposição de $1 \in R$ em uma soma de idempotentes ortogonais com respeito à decomposição $R=P \oplus Q$. Então

$$
u+v=\overline{1}=\pi(1)=\pi(e+f)=\bar{e}+\bar{f},
$$

$\log \bar{e}=u$. Portanto, idempotentes de $S$ podem ser levantados.

Agora, resta mostrar que o anel $\bar{R}=S$ é artiniano. Pelo teorema (1.26), isso é o mesmo que mostrar que ${ }_{S} S$ é completamente redutível, ou seja, que todo $S$-submódulo de $S$ é um somando direto.

Considere um $S$-módulo cíclico ${ }_{S} M$. Então $M$ é cíclico como $R$-módulo, logo tem uma cobertura projetiva sobre $R$ por hipótese. Pelo lema (2.49), ${ }_{S} M$ tem uma cobertura projetiva sobre $S$. Como $J(S)=0$, pelo lema (2.50) temos que ${ }_{S} M$ é projetivo. Em suma, todo $S$-módulo cíclico é projetivo. Considere agora um $S$-submódulo qualquer $L \subseteq S$. O $S$-módulo $S / L$ é gerado por $\overline{1}+L, \operatorname{logo}$ é projetivo. Então, a seqüência exata de $S$-módulos

$$
0 \longrightarrow L \longrightarrow S \longrightarrow S / L \longrightarrow 0
$$

cinde, ou seja, $L$ é um somando direto de $S$ como queríamos demonstrar.

Como veremos, vale o resultado análogo para anéis perfeitos.

Lema 2.52. Seja $M$ um R-módulo. Se $M$ admite uma cobertura projetiva $\theta: P \rightarrow M$, então $\theta(\operatorname{rad} P)=\operatorname{rad} M$.

Prova. Primeiramente observe que $P$ possui submódulos maximais pois, pela proposição $(2.43)$, temos $\operatorname{rad} P \neq P$.

Suponha que $A \subsetneq P$ é um submódulo maximal de $P$, e vejamos que $\theta(A)$ é um submódulo maximal de $M$. Pelo lema (2.45), existe $m \in M$ tal que 
$m \notin \theta(A)$; então $m=\theta(p)$ para algum $p \in P$ tal que $p \notin A$. Como $A$ é maximal, $A+R p=P$. Segue que $\theta(A)+R m=M$, $\log 0 \theta(A)$ é maximal.

Reciprocamente, suponha que $B \subsetneq M$ é um submódulo maximal de $M$, e vejamos que $\theta^{-1}(B)$ é um submódulo maximal de $P$. Pelo lema (2.45), existe $p \in P$ tal que $p \notin \theta^{-1}(B)$; então $\theta(p) \notin B$. Como $B$ é maximal, $B+R \theta(p)=M$. Pelo lema (3.36)(ii) temos que $\theta\left(\theta^{-1}(B)\right)=B \cap \operatorname{im} \theta=B$, $\log \theta \theta\left(\theta^{-1}(B)\right)+\theta(R p)=M$. Portanto, podemos escrever

$$
\theta\left(\theta^{-1}(B)+R p\right)=M .
$$

Do lema (2.45) segue que $\theta^{-1}(B)+R p=P, \log \theta^{-1}(B)$ é maximal.

Vimos, portanto, que $\theta$ estabelece uma correspondência biunívoca entre os submódulos maximais de $P$ e $M$. Em particular, $\theta(\operatorname{rad} P)=\operatorname{rad} M$.

Teorema 2.53 (caracterização homológica de anéis perfeitos). $R e ́$ um anel perfeito à esquerda se, e somente se, todo $R$-módulo admite uma cobertura projetiva.

Prova. Suponha que todo $R$-módulo tem cobertura projetiva. Pelo teorema (2.51) $R$ é semiperfeito, restando provar que $J=J(R)$ é $T$-nilpotente à esquerda.

Seja $M \neq 0$ um $R$-módulo qualquer. $M$ tem uma cobertura projetiva $\theta: P \rightarrow M$ por hipótese, $\operatorname{logo} \theta(\operatorname{rad} P)=\operatorname{rad} M$ por (2.52). Pela proposição (2.43) temos $\operatorname{rad} P=J P \subsetneq P, \log o \theta(\operatorname{rad} P) \neq M$ pelo lema (2.45). Então, $\operatorname{rad} M=J M \neq M$. Portanto, provamos que $J M=M \Longrightarrow M=0$. Pelo lema (2.34), $J$ é $T$-nilpotente à esquerda.

Reciprocamente, se $R$ é perfeito à esquerda então todo $R$-módulo tem cobertura projetiva pelo teorema (2.48).

Note que a prova acima pode ser adaptada para o caso de anéis perfeitos à direita, concluindo assim a caracterização de anéis perfeitos.

A seguir obteremos mais uma caracterização de anéis perfeitos à esquerda, que será feita em termos de módulos planos.

Lema 2.54. Seja $\mathcal{E}: 0 \rightarrow K \rightarrow F \rightarrow M \rightarrow 0$ uma seqüência exata de $R$ módulos e $\mathcal{E}^{\prime}: 0 \rightarrow K^{\prime} \rightarrow F^{\prime} \rightarrow M^{\prime} \rightarrow 0$ uma seqüência exata de módulos- $R$.

(i) Se F é plano e a seqüência $M^{\prime} \otimes_{R} \mathcal{E}$ é exata, então $\mathcal{E}^{\prime} \otimes_{R} M$ é exata.

(ii) Se $F^{\prime}$ é plano e a seqüência $\mathcal{E}^{\prime} \otimes_{R} M$ é exata, então $M^{\prime} \otimes_{R} \mathcal{E}$ é exata. 
Prova. (i) Por hipótese e pela proposição (1.48), o diagrama comutativo abaixo tem colunas e linhas exatas $\left(\otimes\right.$ significa $\left.\otimes_{R}\right)$ :

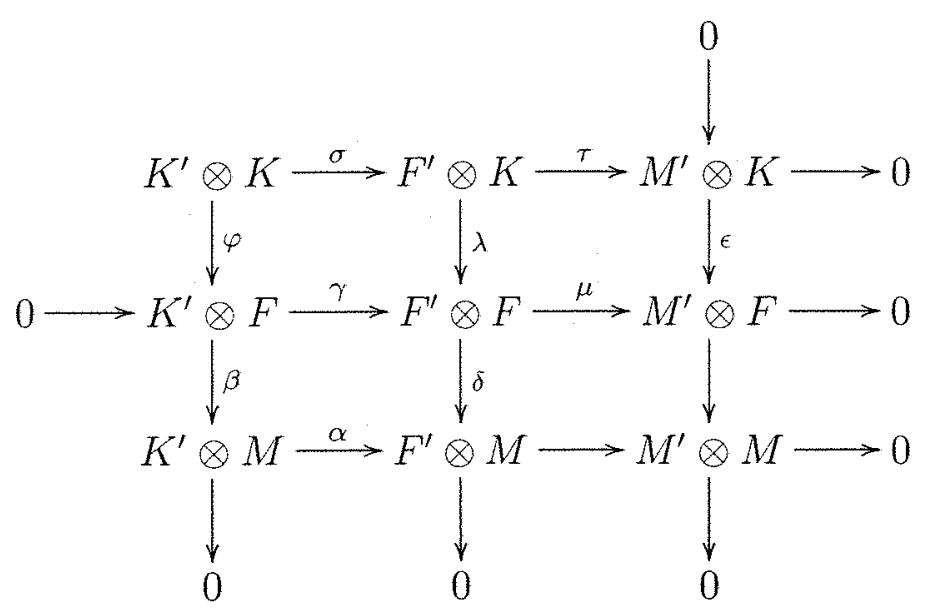

Temos que provar que $\alpha: K^{\prime} \otimes_{R} M \rightarrow F^{\prime} \otimes_{R} M$ é um monomorfismo. Se $x \in K^{\prime} \otimes_{R} M$ é tal que $\alpha(x)=0$, existe $y \in K^{\prime} \otimes_{R} F$ tal que $\beta(y)=x$, portanto $\delta(\gamma(y))=0$ por comutatividade. Como $\operatorname{ker} \delta=\operatorname{im} \lambda, \gamma(y)=\lambda(z)$ para algum $z \in F^{\prime} \otimes_{R} K$. A segunda linha é exata, donde $\mu(\gamma(y))=0$; então $\mu(\gamma(y))=\mu(\lambda(z))=\epsilon(\tau(z))=0$. Temos que $\epsilon$ é mono, logo $\tau(z)=0$. Assim $z \in \operatorname{ker} \tau=\operatorname{im} \sigma$, donde $z=\sigma(w)$ para algum $w \in K^{\prime} \otimes_{R} K$. Finalmente, temos que $\gamma(y)=\lambda(z)=\lambda(\sigma(w))=\gamma(\varphi(w))$. Como $\gamma$ é mono, $y=\varphi(w) \mathrm{e}$ portanto $x=\beta(y)=\beta(\varphi(w))$.

(ii) Pode ser provado por "diagram chasing" como foi feito em $(i)$.

Lema 2.55. Seja $\mathcal{E}: 0 \rightarrow K \rightarrow F \rightarrow M \rightarrow 0$ uma seqüência exata de $R$-módulos, onde ${ }_{R} F$ é plano. Então ${ }_{R} M$ é plano se, e somente se, $M^{\prime} \otimes_{R} \mathcal{E}$ é uma seqüência exata para todo módulo $M_{R}^{\prime}$.

Prova. Em primeiro lugar, suponha que ${ }_{R} M$ é plano. Todo módulo- $R$ unitário é imagem homomorfa de um módulo- $R$ livre, e todo módulo livre é plano. Assim, para qualquer $M_{R}^{\prime}$ dado, existe uma seqüência exata de módulos- $R$

$$
\mathcal{E}^{\prime}: 0 \longrightarrow K^{\prime} \longrightarrow F^{\prime} \longrightarrow M^{\prime} \longrightarrow 0
$$

$\operatorname{com} F_{R}^{\prime}$ um módulo plano. Como ${ }_{R} M$ é plano, $\mathcal{E}^{\prime} \otimes_{R} M$ é exata por definição, portanto $M^{\prime} \otimes_{R} \mathcal{E}$ é exata pelo lema (2.54).

Reciprocamente, suponha que $M^{\prime} \otimes_{R} \mathcal{E}$ é exata para qualquer $M_{R}^{\prime}$ e considere uma seqüencia exata qualquer de módulos- $R$

$$
\mathcal{E}^{\prime}: 0 \longrightarrow K^{\prime} \longrightarrow F^{\prime} \longrightarrow M^{\prime} \longrightarrow 0
$$

Como $M^{\prime} \otimes_{R} \mathcal{E}$ é exata e ${ }_{R} F$ é plano, pelo lema (2.54) temos que $\mathcal{E}^{\prime} \otimes_{R} M$ é exata. Por ser $\mathcal{E}^{\prime}$ arbitrária, ${ }_{R} M$ é plano por definição. 
Proposição 2.56. Sejam $\left\{a_{1}, a_{2}, \ldots\right\} \subseteq R$ uma seqüência dada de elementos de $R,{ }_{R} F$ o módulo livre $\bigoplus_{i=0}^{\infty} R e_{i}$ e $K \subseteq F$ o submódulo livre gerado por $\left\{f_{i}=e_{i}-a_{i+1} e_{i+1}: i \geqslant 0\right\}$. Então o R-módulo $M=F / K$ é plano, e se $M$ é projetivo então a cadeia descendente $a_{1} R \supseteq a_{1} a_{2} R \supseteq \cdots$ de ideais à direita principais estabiliza.

Prova. Pelo lema (2.55) e pelo corolário (1.49), para provar que ${ }_{R} M$ é plano basta mostrar que a aplicação de inclusão (id $\otimes \iota$ ) :X $X \otimes_{R} K \rightarrow X \otimes_{R} F$ é um monomorfismo para qualquer módulo $X_{R}$. Para tanto, devemos mostrar que $($ id $\otimes \iota)(\alpha) \in X \otimes_{R} F$ é zero somente se $\alpha \in X \otimes_{R} K$ é zero. Podemos escrever

$$
X \otimes_{R} K=\bigoplus_{i=0}^{\infty}\left(X \otimes f_{i}\right) \quad \text { e } \quad X \otimes_{R} F=\bigoplus_{i=0}^{\infty}\left(X \otimes e_{i}\right) ;
$$

se $\alpha=\sum_{i=0}^{n} x_{i} \otimes f_{i}$ é tal que (id $\left.\otimes \iota\right)(\alpha) \in X \otimes_{R} F$ é zero, então

$$
\begin{aligned}
0 & =x_{0} \otimes\left(e_{0}-a_{1} e_{1}\right)+\cdots+x_{n} \otimes\left(e_{n}-a_{n+1} e_{n+1}\right) \\
& =x_{0} \otimes e_{0}+\left(x_{1}-x_{0} a_{1}\right) \otimes e_{1}+\cdots+\left(x_{n}-x_{n-1} a_{n}\right) \otimes e_{n}-x_{n} a_{n+1} \otimes e_{n+1} .
\end{aligned}
$$

Daí $x_{0}=x_{1}=\cdots=x_{n}=0$, portanto $\alpha=0$, provando que $M$ é plano.

Suponha, agora, que $M$ é projetivo. Então a seqüencia exata

$$
0 \longrightarrow K \longrightarrow F \longrightarrow M \longrightarrow 0
$$

cinde, de modo que existe um epimorfismo $\pi: F \rightarrow K$ tal que $\pi \circ \iota=\mathrm{id}_{K}$ (como antes, $\iota: K \rightarrow F$ é inclusão). Defina

$$
\pi\left(e_{i}\right)=\sum_{j=0}^{\infty} b_{i j} f_{j}
$$

onde apenas um número finito de coeficientes $b_{i j} \in R$ são diferentes de zero para cada $i$ dado. Então

$$
f_{i}=\pi\left(f_{i}\right)=\pi\left(e_{i}-a_{i+1} e_{i+1}\right)=\sum_{j=0}^{\infty} b_{i j} f_{j}-\sum_{j=0}^{\infty} a_{i+1} b_{i+1, j} f_{j} .
$$

Como $K$ é livre, comparando coeficientes obtemos

$$
b_{i i}-a_{i+1} b_{i+1, i}=1 \quad \text { e } \quad b_{i j}-a_{i+1} b_{i+1, j}=0
$$


para todos $i, j$ com $i \neq j$. Portanto, podemos escrever

$$
\begin{aligned}
& b_{0 j}=a_{1} b_{1 j} \\
b_{1 j}=a_{2} b_{2 j} & \Longrightarrow a_{1} b_{1 j}=a_{1} a_{2} b_{2 j} \\
b_{2 j}=a_{3} b_{3 j} & \Longrightarrow a_{1} a_{2} b_{2 j}=a_{1} a_{2} a_{3} b_{3 j} \\
& \cdots \\
b_{j-1, j}=a_{j} b_{j, j} & \Longrightarrow a_{1} a_{2} \cdots a_{j-1} b_{j-1, j}=a_{1} a_{2} \cdots a_{j} b_{j, j} .
\end{aligned}
$$

Para $j$ suficientemente grande temos $b_{0 j}=0$, logo

$$
0=b_{0 j}=a_{1} b_{1 j}=a_{1} a_{2} b_{2 j}=\cdots=a_{1} a_{2} \cdots a_{j} b_{j j} .
$$

Finalmente, pelas equações (2.5) e (2.6) podemos escrever

$$
\begin{aligned}
a_{1} a_{2} \cdots a_{j} & =a_{1} a_{2} \cdots a_{j}-a_{1} a_{2} \cdots a_{j} b_{j j} \\
& =a_{1} a_{2} \cdots a_{j}\left(1-b_{j j}\right) \\
& =a_{1} a_{2} \cdots a_{j} a_{j+1}\left(-b_{j+1, j}\right)
\end{aligned}
$$

Se $x \in a_{1} a_{2} \cdots a_{j} R$ então, para algum $r \in R$, temos

$$
x=a_{1} a_{2} \cdots a_{j} r=a_{1} a_{2} \cdots a_{j} a_{j+1}\left(-b_{j+1, j}\right) r \in a_{1} a_{2} \cdots a_{j} a_{j+1} R,
$$

logo $a_{1} a_{2} \cdots a_{j} R \subseteq a_{1} a_{2} \cdots a_{j} a_{j+1} R$ para algum índice $j$ suficientemente grande. Isso significa que a cadeia descendente $a_{1} R \supseteq a_{1} a_{2} R \supseteq \cdots$ de ideais à direita principais estabiliza.

Lema 2.57. Sejam $J=J(R), \bar{R}=R / J$ e $K$ um R-módulo qualquer. Então $\bar{R} \otimes_{R} K$ e K/JK são isomorfos como $R$-módulos.

Prova. É rotina verificar que a aplicação $\varphi: K / J K \rightarrow \bar{R} \otimes_{R} K$ definida por $k+J K \mapsto \overline{1} \otimes k$ está bem definida e é $R$-homomorfismo. Considere, agora, a aplicação $f: \bar{R} \times K \rightarrow K / J K$ definida por $(\bar{r}, k) \mapsto r k+J K$. Esta aplicação está bem definida e é $R$-balanceada; logo, pela propriedade universal do produto tensorial, existe um único homomorfismo $F: \bar{R} \otimes_{R} K \rightarrow K / J K$ de grupos abelianos tal que o diagrama abaixo é comutativo:

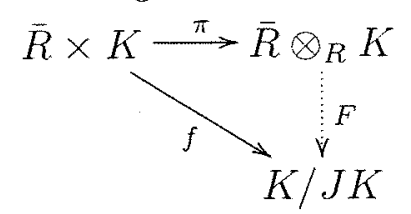

É fácil ver, também, que $F$ é um $R$-homomorfismo e que é definida por

$$
\bar{r} \otimes k \longmapsto r k+J K \text {. }
$$


Assim, $\varphi \circ F$ e $F \circ \varphi$ são aplicações identidade, donde $\bar{R} \otimes_{R} K$ e $K / J K$ são isomorfos como $R$-módulos.

O resultado abaixo completa a prova do teorema (2.37) e oferece uma caracterização homológica de anéis perfeitos que será de grande importância no próximo capítulo.

Teorema 2.58 (Bass - 1960). Para um anel qualquer $R$, sâo equivalentes:

(i) $R$ é perfeito à esquerda.

(ii) R satisfaz a CCD em ideais à direita principais.

(iii) Todo módulo $N_{R}$ satisfaz a CCD em submódulos cíclicos.

(iv) $R$ não contém uma família ortogonal infinita de idempotentes nãanulos, e todo módulo não-nulo $N_{R}$ contém um submódulo simples.

(v) Todo R-módulo plano é projetivo.

Prova. Na demonstração do teorema (2.37), provamos apenas as implicações $($ ii $) \Rightarrow($ iii $) \Rightarrow(i v) \Rightarrow(i)$. Para concluir a demonstração, provaremos que $(i) \Rightarrow(v) \Rightarrow(i i)$.

$(i) \Rightarrow(v)$ Seja $M$ um $R$-módulo plano. Pelo teorema (2.48), $M$ tem uma cobertura projetiva $\theta: P \rightarrow M$. Podemos, assim, considerar a seqüência exata de $R$-módulos

$$
\mathcal{E}: 0 \longrightarrow K \longrightarrow P \longrightarrow M \longrightarrow 0,
$$

sendo $K=\operatorname{ker} \theta \subseteq_{s} P$. Pelo lema $(2.55), M^{\prime} \otimes_{R} \mathcal{E}$ é um seqüência exata para todo módulo $M_{R}^{\prime}$. Em particular, se escolhemos $M_{R}^{\prime}=R / J(R)=\bar{R}$ então

$$
\bar{R} \otimes_{R} K \longrightarrow \bar{R} \otimes_{R} P
$$

é um monomorfismo; portanto, pelo lema (2.57), a aplicação induzida

$$
\alpha: \frac{K}{J K} \longrightarrow \frac{P}{J P}
$$

dada por $k+J K \longmapsto k+J P$, é um monomorfismo. No entanto, pelas proposições (2.41) e (2.43), $K \subseteq_{s} P$ implica que $K \subseteq \operatorname{rad} P=J P$; portanto $\alpha$ é a aplicação nula, donde se conclui que $K=J K$. Como $R$ é perfeito à esquerda por hipótese, $J$ é $T$-nilpotente à esquerda; do lema (2.34) segue, portanto, que $K=0$. Logo, $M \simeq P$ é projetivo. 
$(v) \Rightarrow($ ii $)$ Se todo $R$-módulo plano é projetivo, considere uma cadeia descendente qualquer $a_{1} R \supseteq a_{1} a_{2} R \supseteq \cdots$ de ideais à direita principais. Tal como foi feito na proposição $(2.56)$, podemos associar à seqüência $\left\{a_{1}, a_{2}, \ldots\right\}$ um $R$-módulo plano $M$ que, por hipótese, é também projetivo. A conclusão da proposição (2.56) é que a cadeia $a_{1} R \supseteq a_{1} a_{2} R \supseteq \cdots$ estabiliza.

Observe que a demonstração do teorema de Bass teve que passar pela condição homológica $(v)$ acima. No exemplo (2.67) da próxima seção vemos um módulo projetivo que não é plano (sobre um anel que não é perfeito).

Para finalizar, observamos que é possível uma demonstração direta de (i) $\Rightarrow($ ii $)$, sem utilizar conceitos de Álgebra Homológica. Tal demonstração foi realizada por R. Rentschler; para uma exposição da demonstração de Rentschler, indicamos ao leitor o livro de Rowen [RO II].

\subsection{Exemplos}

Nesta seção, serão apresentados alguns exemplos que ilustram a cadeia de inclusões entre as classes de anéis consideradas neste capítulo:

artinianos $\subsetneq$ semiprimários $\subsetneq$ perfeitos $\subsetneq$ semiperfeitos $\subsetneq$ semilocais.

Note que os conceitos "artiniano" e "perfeito" admitem uma distinção entre esquerda e direita, enquanto as outras denominações são simétricas.

Proposição 2.59. Todo anel artiniano é semiprimário.

Prova. Se $R$ é artiniano então $R / J(R)$ é artiniano e $J(R)$ é nilpotente pelo teorema (1.24), logo $R$ é semiprimário.

Proposição 2.60. Todo anel semiprimário é perfeito.

Prova. Se $R$ é um anel semiprimário então $R / J(R)$ é artiniano e $J(R)$ é nilpotente. É claro que todo ideal nilpotente é $T$-nilpotente à esquerda e à direita, logo $R$ é perfeito.

Proposição 2.61. Todo anel perfeito (à esquerda ou à direita) é um anel semiperfeito.

Prova. Se $R$ é um anel perfeito à esquerda, então $R / J(R)$ é artiniano e $J(R)$ é um ideal $T$-nilpotente à esquerda. Daí $J(R)$ é um ideal nil, e idempotentes de $R / J(R)$ podem ser levantados pela proposição $(2.9)$. 
Por definição, é claro que anéis semiperfeitos são semilocais. Veremos agora uma pequena série de exemplos que mostram que as inclusões entre essas classes de anéis são, de fato, próprias. O primeiro exemplo trata de um anel de frações.

\section{Exemplo 2.62 (um anel semilocal que não é semiperfeito).}

Seja $n$ um produto de números primos distintos $p_{1}, \ldots, p_{t}$ e seja

$$
\pi: \mathbb{Z} \longrightarrow \mathbb{Z}_{n}=\mathbb{Z} / n \mathbb{Z}
$$

a projeção canônica. Defina $S=\{m \in \mathbb{Z}:(m, n)=1\}$ e considere o anel de frações $R=S^{-1} \mathbb{Z}$. Como os elementos de $\pi(S)$ são invertíveis, podemos estender $\pi$ a um epimorfismo

$$
\sigma: R \longrightarrow \mathbb{Z}_{n}
$$

definido por $\sigma\left(s^{-1} m\right)=[\pi(s)]^{-1} \pi(m)$ para todos $s \in S, m \in \mathbb{Z}$. Observe que ker $\sigma=n R$ é um ideal quase-regular de $R$, logo ker $\sigma \subseteq J(R)$ pelo teorema (1.24). Do primeiro teorema do isomorfismo temos $R /$ ker $\sigma \simeq \mathbb{Z}_{n}$; como $\mathbb{Z}_{n}$ é artiniano semisimples temos $J(R / \operatorname{ker} \sigma)=0, \operatorname{logo} J(R) \subseteq \operatorname{ker} \sigma$, novamente por (1.24). Então $J(R)=\operatorname{ker} \sigma, \bar{R}=R / J(R) \simeq \mathbb{Z}_{n}$ e $R$ é um anel semilocal. Do teorema chinês dos restos temos

$$
\mathbb{Z}_{n} \simeq \frac{\mathbb{Z}}{p_{1} \mathbb{Z}} \oplus \cdots \oplus \frac{\mathbb{Z}}{p_{t} \mathbb{Z}}
$$

logo $\bar{R}$ tem idempotentes não-triviais se $t \geqslant 2$. No entanto $R$ é um subanel de $\mathbb{Q}, \operatorname{logo} R$ só possui os idempotentes triviais. Então não é possivel levantar todos os idempotentes de $\vec{R}$, donde concluímos que $R$ não é semiperfeito.

\section{Exemplo 2.63 (um anel semiperfeito que não é perfeito).}

Seja $K$ um corpo e $S=K[[x]]$ o anel de séries de potências formais na indeterminada $x$. Então $S$ é um anel comutativo local com ideal maximal $J=J(S)=S x$. Pelo teorema (2.30), o anel de matrizes $R=\mathbb{M}_{n}(S)$ é um anel semiperfeito com quociente simples pelo seu radical. Todo ideal próprio de $S$ é da forma $S x^{t}$ para algum inteiro positivo $t$, logo todo ideal próprio de $R$ é da forma $\mathbb{M}_{n}\left(S x^{t}\right)$ para algum $t$. Logo, $J(R)=\mathbb{M}_{n}(S x)$. É claro que $J(R)$ não é nil, portanto $J(R)$ não é $T$-nilpotente. Daí, $R$ não é perfeito.

No exemplo acima note que, apesar de $J(R)$ não ser um ideal nil, ainda é possível levantar idempotentes de $J(R)$. Isso mostra que a condição da proposição (2.9) não é necessária. 


\section{Exemplo 2.64 (um anel perfeito à direita mas não à esquerda).}

Sejam $F$ um corpo e $V$ um espaço vetorial sobre $F$ com base $\left\{e_{1}, e_{2}, \ldots\right\}$. A álgebra $\operatorname{End}_{F}(V)$ pode ser vista como uma álgebra de "matrizes infinitas" com entradas em $F$, e os elementos $e_{i}$ da base de $V$ podem ser vistos como matrizes-coluna. Seja $J \subseteq \operatorname{End}_{F}(V)$ o conjunto de matrizes infinitas sobre $F$ que têm um número finito de entradas não-nulas, todas ocorrendo acima da diagonal principal, e considere a subálgebra $R=F \cdot \mathbf{1}+J \subseteq \operatorname{End}_{F}(V)$, onde 1 denota a identidade de $\operatorname{End}_{F}(V)$. É fácil ver que $J$ é um ideal de $R$ e que $R / J \simeq F$. Para verificar que $J$ é $T$-nilpotente à direita, considere uma seqüência qualquer $\left\{a_{1}, a_{2}, \ldots\right\} \subseteq J$. Pela definição de $J$, existe um inteiro positivo $n$ tal que multiplicação à esquerda por $a_{1}$ anula todos os elementos $e_{n+1}, e_{n+2}, \ldots$ da base de $V$. Escreva $V_{i}=e_{1} F \oplus e_{2} F \oplus \cdots \oplus e_{i} F$ para todo $i \geqslant 1$; um cálculo simples mostra que

$$
a_{1} V_{n} \subseteq V_{n-1} \quad a_{2} a_{1} V_{n} \subseteq V_{n-2} \quad a_{3} a_{2} a_{1} V_{n} \subseteq V_{n-3} \quad \ldots
$$

donde $a_{n} \cdots a_{2} a_{1} V_{n}=0$. Então $a_{n} \cdots a_{2} a_{1} V_{i}=0$ para todo $i \geqslant 1$, donde concluímos que $a_{n} \cdots a_{2} a_{1} V=0$. Portanto $a_{n} \cdots a_{2} a_{1}=0$ e assim vemos que $J$ é $T$-nilpotente à direita. Então $J$ é nil, donde $J \subseteq J(R)$. Como $R / J \simeq F$ temos $J(R) \subseteq J$, portanto $J$ é o radical de $R$ e $R$ é um anel local, perfeito à direita. No entanto, $J$ não é $T$-nilpotente à esquerda. De fato, tome a seqüência $\left\{a_{1}, a_{2}, \ldots\right\} \subseteq J$ tal que $a_{i}$ é a matriz infinita elementar $E_{i, i+1}$ e note que

$$
a_{1} a_{2}=E_{13} \quad a_{1} a_{2} a_{3}=E_{14} \quad a_{1} a_{2} a_{3} a_{4}=E_{15} \quad \ldots
$$

Portanto $a_{1} a_{2} \cdots a_{n} \neq 0$, não importa quão grande seja $n$. Logo, $R$ não é perfeito à esquerda.

Uma conseqüência deste exemplo é o fato de que ideais $T$-nilpotentes não são necessariamente nilpotentes.

Exemplo 2.65 (um anel perfeito à direita, mas não semiprimário).

Basta verificar que o anel $R$ do exemplo acima tem um radical $J(R)$ que é $T$-nilpotente à direita, mas que não é nilpotente por não ser $T$-nilpotente à esquerda.

\section{Exemplo 2.66 (um anel semiprimário que não é artiniano).}

Considere o anel $R=\left[\begin{array}{cc}\mathbb{Q} & \mathbb{R} \\ 0 & \mathbb{Q}\end{array}\right]$, cujo radical é $J(R)=\left[\begin{array}{cc}0 & \mathbb{R} \\ 0 & 0\end{array}\right] . J(R)$ é nilpotente e $R / J(R) \simeq \mathbb{Q} \oplus \mathbb{Q}$ é artiniano, logo $R$ é semiprimário. Como $\mathbb{R}$ 
é um espaço vetorial de dimensão infinita sobre $\mathbb{Q}$, podemos construir uma cadeia ascendente infinita de extensões

$$
\mathbb{Q} \subsetneq K_{1} \subsetneq K_{2} \subsetneq \cdots,
$$

sendo cada $K_{n}$ de dimensão finita sobre $\mathbb{Q}$. Então podemos construir uma cadeia ascendente infinita

$$
\left[\begin{array}{ll}
0 & \mathbb{Q} \\
0 & 0
\end{array}\right] \subsetneq\left[\begin{array}{cc}
0 & K_{1} \\
0 & 0
\end{array}\right] \subsetneq\left[\begin{array}{cc}
0 & K_{2} \\
0 & 0
\end{array}\right] \cdots
$$

de ideais bilaterais de $R$, e portanto $R$ não é noetheriano (nem à esquerda, nem à direita). Pelo teorema de Hopkins-Levitzki, $R$ não é artiniano.

No teorema de Bass (2.58), vimos que se $R$ é perfeito à esquerda então todo $R$-módulo plano é projetivo. O próximo exemplo mostra um módulo (sobre um anel que não é perfeito) que é plano mas não é projetivo.

\section{Exemplo 2.67 (um módulo plano que não é projetivo).}

Considere o módulo $\mathbb{Z} \mathbb{Q}$.

Para ver que $\mathbb{Z} \mathbb{Q}$ é plano, suponha que $\varphi: L \rightarrow M$ é um monomorfismo de $\mathbb{Z}$-módulos. Todo elemento de $\mathbb{Q} \otimes_{\mathbb{Z}} L$ pode ser escrito na forma $(1 / d) \otimes \ell$ para algum inteiro $d \neq 0$ e algum $\ell \in L$. Se $(1 / d) \otimes \ell$ está no núcleo de $1 \otimes \varphi$, então $(1 / d) \otimes \varphi(\ell)=0 \mathrm{em} \mathbb{Q} \otimes_{\mathbb{Z}} M$. Isso significa que $n \varphi(\ell)=0$ para algum inteiro $n \neq 0$. Como $\varphi$ é mono temos $n \ell=0$, e portanto $(1 / d) \otimes \ell=(n / d) \otimes n \ell=0$. Logo $1 \otimes \varphi$ é injetora, donde $\mathbb{Z}_{\mathbb{Q}}$ é plano pelo corolário (1.49).

Suponha que $\mathbb{Z} \mathbb{Q}$ é projetivo. Pela proposição (1.44) temos que $\mathbb{Z} \mathbb{Q}$ é somando direto de algum $\mathbb{Z}$-módulo livre $F$, ou seja, existe um $\mathbb{Z}$-módulo $K$ tal que $F=\mathbb{Q} \oplus K$. No entanto, observe que $\mathbb{Q}=n \mathbb{Q}$ para todo inteiro $n \neq 0$ (um $\mathbb{Z}$-módulo com essa propriedade é chamado divisivel). Como $F$ é livre, é fácil ver que

$$
\bigcap_{n \in \mathbb{Z} \backslash\{0\}} n F=0,
$$

mas

$$
\bigcap_{n \in \mathbb{Z} \backslash\{0\}} n \mathbb{Q} \oplus n K=\bigcap_{n \in \mathbb{Z} \backslash\{0\}} \mathbb{Q} \oplus n K \neq 0 .
$$

Dessa contradição, vemos que ${ }_{\mathbb{Z}} \mathbb{Q}$ não é projetivo.

Note que isso não contradiz o teorema de Bass, pois $\mathbb{Z}$ não é perfeito à esquerda nem à direita (de fato, $\mathbb{Z}$ não é nem semilocal). O próximo exemplo é um corolário do exemplo anterior. 
Exemplo 2.68 (um módulo que não admite cobertura projetiva).

Novamente, considere o $\mathbb{Z}$-módulo $\mathbb{Z} \mathbb{Q}$. Como $J(\mathbb{Z})=0$, se $\mathbb{Z} \mathbb{Q}$ admitisse cobertura projetiva então seria um módulo projetivo pelo lema (2.50). Como não é esse o caso, $\mathbb{Z} \mathbb{Q}$ não admite cobertura projetiva. 


\section{Capítulo 3}

\section{Anéis e Módulos Distributivos}

Neste capítulo desenvolveremos os conceitos de anel e módulo distributivo. Após uma breve exposição sobre reticulados na seção 3.1, descrevemos uma certa classe de módulos distributivos na seção 3.2 , que é então concluída com o teorema de Behrens (3.30). A seção 3.3 apresenta o teorema de Stephenson (3.46), que generaliza o teorema (3.30) descrevendo anéis distributivos com alguma propriedade adicional, relacionada às condições de finitude estudadas no capítulo 2 .

Os primeiros trabalhos sobre anéis e módulos distributivos no caso nãocomutativo foram desenvolvidos na década de 1960 por Behrens; veja, por exemplo, [BE I] e [BE II]. O estudo sistemático de módulos distributivos sobre anéis não-comutativos foi iniciado na década de 1970 com os artigos [ST I], $[\mathrm{AC}],[\mathrm{CA}]$ e $[\mathrm{BR}]$. Para um estudo exaustivo do assunto, indicamos ao leitor o livro de Tuganbaev [TU].

\subsection{Reticulados}

Em 1854, George Boole (1815 - 1864) criou uma nova classe de estruturas algébricas, chamadas álgebras de Boole, que são tipos especiais de reticulados (lattices). Foi E. Schröder, em torno de 1890, quem primeiro considerou o conceito de reticulado em seu sentido atual. Aproximadamente ao mesmo tempo, Richard Dedekind desenvolveu um conceito similar em seu trabalho sobre grupos e ideais. Dedekind definiu o que chamamos, em terminologia moderna, de reticulados modulares e distributivos. O rápido desenvolvimento da teoria de reticulados começou propriamente na década de 1930, quando Garret Birkhoff realizou grandes contribuições à teoria.

Nossa exposição segue, basicamente, as referências [CO] e [LP]. 
Reticulados podem ser definidos de duas maneiras equivalentes, como veremos imediatamente a seguir.

Definição 3.1. Seja $(A, \preccurlyeq)$ um conjunto parcialmente ordenado $e B \subseteq A$.

(i) $a \in A$ é uma cota superior de $B$ se $b \preccurlyeq a$ para todo $b \in B$.

(ii) $a \in A$ é uma cota inferior de $B$ se $a \preccurlyeq b$ para todo $b \in B$.

(iii) O infimo de $B$, quando existe, é a maior das cotas inferiores de $B$.

(iv) O supremo de B, quando existe, é a menor das cotas superiores de B.

Definição 3.2 (reticulados ordenados). Um reticulado ordenado é um conjunto parcialmente ordenado $(\mathcal{L}, \preccurlyeq)$ tal que, para todo par de elementos $x, y \in \mathcal{L}$, existem em $\mathcal{L}$ o supremo e o ínfimo de $\{x, y\}$.

Reticulados também podem ser definidos de forma puramente algébrica, sem menção explícita a relações de ordem.

Definição 3.3 (reticulados algébricos). Um reticulado algébrico é um conjunto não-vazio $\mathcal{L}$ munido de duas operações binárias $\vee$ e $\wedge$ satisfazendo

$$
\begin{array}{lll}
a \vee a=a & a \wedge a=a & \text { (lei idempotente) } \\
a \vee b=b \vee a & a \wedge b=b \wedge a & \text { (lei comutativa) } \\
a \vee(b \vee c)=(a \vee b) \vee c & a \wedge(b \wedge c)=(a \wedge b) \wedge c & \text { (lei associativa) } \\
a \vee(a \wedge b)=a & a \wedge(a \vee b)=a & \text { (lei de absorção) }
\end{array}
$$

para todos $a, b, c \in \mathcal{L}$.

O teorema abaixo estabelece a equivalência entre as definições dadas.

Teorema 3.4 (reticulados ordenados são algébricos e vice-versa).

(i) Seja $(\mathcal{L}, \preccurlyeq)$ um reticulado ordenado. Se definimos duas operações binárias $\wedge e \vee$ em $\mathcal{L}$ pondo

$$
x \wedge y=\inf \{x, y\} \quad \text { e } \quad x \vee y=\sup \{x, y\}
$$

para todos $x, y \in \mathcal{L}$, então $(\mathcal{L}, \wedge, \vee)$ é um reticulado algébrico.

(ii) Seja $(\mathcal{L}, \wedge, \vee)$ um reticulado algébrico. Se definimos uma relação de ordem em $\mathcal{L}$ pondo

$$
x \preccurlyeq y \Longleftrightarrow x \wedge y=x \Longleftrightarrow x \vee y=y
$$

para todos $x, y \in \mathcal{L}$, então $(\mathcal{L}, \preccurlyeq)$ é um reticulado ordenado. 


\section{Prova.}

(i) Seja $(\mathcal{L}, \preccurlyeq)$ um reticulado ordenado. Para $x, y, z \in \mathcal{L}$ arbitrários, é claro que $x \wedge x=x=x \vee x$ e que $x \wedge y=y \wedge x, x \vee y=y \vee x$, logo valem as leis idempotente e comutativa. Temos agora

$$
\begin{aligned}
x \wedge(y \wedge z) & =x \wedge \inf \{y, z\}=\inf \{x, \inf \{y, z\}\}=\inf \{x, y, z\} \\
& =\inf \{\inf \{x, y\}, z\}=\inf \{x, y\} \wedge z=(x \wedge y) \wedge z
\end{aligned}
$$

analogamente, segue que $x \vee(y \vee z)=(x \vee y) \vee z$, logo vale a lei associativa. Finalmente,

$$
\begin{aligned}
& x \wedge(x \vee y)=x \wedge \sup \{x, y\}=\inf \{x, \sup \{x, y\}\}=x, \\
& x \vee(x \wedge y)=x \vee \inf \{x, y\}=\sup \{x, \inf \{x, y\}\}=x,
\end{aligned}
$$

logo vale a lei de absorção. Assim, $(\mathcal{L}, \preccurlyeq)$ é um reticulado algébrico.

(ii) Seja $(\mathcal{L}, \wedge, \vee)$ um reticulado algébrico. Se $x, y, z \in \mathcal{L}$ são elementos arbitrários, temos:

- $x \wedge x=x$ e $x \vee x=x$ pela lei idempotente; logo $x \preccurlyeq x$, ou seja, $\preccurlyeq$ é reflexiva.

- Se $x \preccurlyeq y$ e $y \preccurlyeq x$ então $x \wedge y=x$ e $y \wedge x=y$. Pela lei comutativa, temos $x=y$, ou seja, $\preccurlyeq$ é anti-simétrica.

- Se $x \preccurlyeq y$ e $y \preccurlyeq z$ então $x \wedge y=x$ e $y \wedge z=y$. Pela lei associativa temos

$$
x=x \wedge y=x \wedge(y \wedge z)=(x \wedge y) \wedge z=x \wedge z,
$$

daí $x \preccurlyeq z$, ou seja, ß́ é transitiva.

Mostramos, assim, que $\preccurlyeq$ é uma relação de ordem parcial em $\mathcal{L}$, restando mostrar que supremos e ínfimos sempre existem.

Sejam $x, y \in \mathcal{L}$. Então $x \wedge(x \vee y)=x$ (lei de absorção) implica $x \preccurlyeq x \vee y$, e analogamente temos $y \preccurlyeq x \vee y$. Se $z \in \mathcal{L}$ é tal que $x \preccurlyeq z$ e $y \preccurlyeq z$, então $(x \vee y) \vee z=x \vee(y \vee z)=x \vee z=z, \log 0 x \vee y \preccurlyeq z, \mathrm{e} \operatorname{assim} \sup \{x, y\}=x \vee y$. Analogamente prova-se também que inf $\{x, y\}=x \wedge y$. Assim, $(\mathcal{L}, \wedge, \vee)$ é um reticulado ordenado.

Em vista do resultado acima, não mais faremos distinção entre reticulados ordenados ou algébricos, considerando a compatibilidade entre as estruturas sempre da forma como foi definida no teorema (3.4).

O exemplo mais natural de reticulado é $\mathcal{P}(C)$, o conjunto das partes de um dado conjunto $C$, sendo $\vee$ e $\wedge$ a união e a interseção de conjuntos $\mathrm{e} \preccurlyeq \mathrm{a}$ 
ordenação parcial por inclusão. Outro exemplo, de grande importância, é o reticulado $L_{R}(M)$ de $R$-submódulos de um $R$-módulo $M$, com as operações de soma e interseção de submódulos e ordenação parcial por inclusão.

Lema 3.5 (monotonicidade). Seja $\mathcal{L}$ um reticulado. Se $a, b, c \in \mathcal{L}$ e $a \preccurlyeq b$ então

$$
(a \wedge c) \preccurlyeq(b \wedge c) \quad e \quad(a \vee c) \preccurlyeq(b \vee c)
$$

Prova. $(a \wedge c) \wedge(b \wedge c)=a \wedge c$ e $(a \vee c) \vee(b \vee c)=b \vee c$

Para desenvolver nossa linguagem de reticulados, precisaremos de uma série de definições.

Definição 3.6 (limites). Seja $\mathcal{L}$ um reticulado. Se $\mathcal{L}$ contém um elemento máximo com respeito a unidade e é denotado por 1 . Similarmente, se $\mathcal{L}$ tem um elemento mínimo então esse elemento é chamado de elemento nulo e é denotado por 0 . Esses elementos são chamados limites de $\mathcal{L}$, e dizemos que $\mathcal{L}$ é limitado.

Definição 3.7 (subreticulados, homomorfismos, intervalos, cadeias). Seja $\mathcal{L}$ um reticulado. Um subconjunto $\mathcal{S} \subseteq \mathcal{L}$ é dito um subreticulado de $\mathcal{L}$ se $\mathcal{S}$ é um reticulado.

$\operatorname{Sejam}\left(\mathcal{L}_{1}, \wedge_{1}, \vee_{1}\right)$ e $\left(\mathcal{L}_{2}, \wedge_{2}, \vee_{2}\right)$ dois reticulados. Um homomorfismo de reticulados é uma aplicação $\varphi: \mathcal{L}_{1} \rightarrow \mathcal{L}_{2}$ tal que

$$
\varphi\left(x \vee_{1} y\right)=\varphi(x) \vee_{2} \varphi(y) \quad e \quad \varphi\left(x \wedge_{1} y\right)=\varphi(x) \wedge_{2} \varphi(y)
$$

para todos $x, y \in \mathcal{L}_{1}$. Isomorfismos são definidos da maneira usual.

Se $a, b \in \mathcal{L}$ e $a \preccurlyeq b$, o subconjunto $[a, b]=\{x \in \mathcal{L}: a \preccurlyeq x \preccurlyeq b\}$ é ditoo intervalo definido por a e b. É fácil ver que todo intervalo é um subreticulado. Uma cadeia é um intervalo totalmente ordenado em $\mathcal{L}$.

Definição 3.8 (complementos). Sejam $\mathcal{L}$ um reticulado $e[u, v] \subseteq \mathcal{L} u m$ intervalo. Dado $a \in[u, v]$, um elemento $b \in[u, v]$ é dito um complemento de a em $[u, v]$ (ou um complemento de a em relação $a[u, v]$ ) se $a \wedge b=u e$ $a \vee b=v$. Se dois elementos $b, c \in[u, v]$ são complementos de a em $[u, v]$, dizemos que b e c são a-relacionados em $[u, v]$.

A linguagem de reticulados é naturalmente apropriada para descrever a estrutura de submódulos de um módulo, ou de ideais de um anel. Por exemplo, se $M$ é um módulo semisimples sobre um anel $R$, dizemos que o reticulado de submódulos de $M$ é complementado, ou seja, todo submódulo $S \subseteq M$ tem um complemento direto no intervalo $[0, M]$. 
Proposição 3.9 (desigualdade modular). Se $\mathcal{L}$ é um reticulado contendo um intervalo $\mathcal{I}=[a, c]$, para todo $b \in \mathcal{L}$ vale a desigualdade

$$
a \vee(b \wedge c) \preccurlyeq(a \vee b) \wedge c .
$$

Prova. Temos $a \preccurlyeq(a \vee b)$ e $a \preccurlyeq c, \log 0$

$$
a \preccurlyeq \inf \{(a \vee b), c\}=(a \vee b) \wedge c ;
$$

temos ainda que $(b \wedge c) \preccurlyeq b \preccurlyeq(a \vee b)$ e $(b \wedge c) \preccurlyeq c$, logo

$$
(b \wedge c) \preccurlyeq \inf \{(a \vee b), c\}=(a \vee b) \wedge c .
$$

Sendo assim, $a \vee(b \wedge c)=\sup \{a,(b \wedge c)\} \preccurlyeq(a \vee b) \wedge c$.

Em geral, temos ao menos duas maneiras distintas de projetar $\mathcal{L}$ sobre o intervalo $\mathcal{I}=[a, c]$ : os operadores de projeçâao $p_{\mathcal{I}}$ e $P_{\mathcal{I}}$ definidos por

$$
p_{\mathcal{I}}(x)=a \vee(x \wedge c) \quad \text { e } \quad P_{\mathcal{I}}(x)=(a \vee x) \wedge c
$$

para todo $x \in \mathcal{L}$. Sendo assim, a desigualdade modular pode ser escrita, mais simplesmente, como

$$
p_{\mathcal{I}}(b) \preccurlyeq P_{\mathcal{I}}(b) \quad \forall \mathcal{I} \subseteq \mathcal{L}, \forall b \in \mathcal{L} .
$$

Veremos agora uma classe de reticulados para os quais sempre vale o sinal de igualdade na desigualdade modular.

Definição 3.10 (reticulados modulares). O reticulado $\mathcal{L}$ é modular se

$$
a \vee(b \wedge c)=(a \vee b) \wedge c
$$

para todos $a, b, c \in \mathcal{L}$ tais que $a \preccurlyeq c$. A equação acima é denominada lei modular.

Um importante exemplo de reticulado modular é o reticulado $L(G)$ de subgrupos normais de um grupo $G$, sendo $\vee$ e $\wedge$ o produto e a interseção de subgrupos, respectivamente. De fato, seja $G$ um grupo e $A, B, C$ subgrupos normais de $G$ com $A \subseteq C$. Para provar que $L(G)$ é modular, basta mostrar que $A B \cap C \subseteq A(B \cap C)$. Se $c \in A B \cap C$, podemos escrever $c=a b \mathrm{com}$ $a \in A$ e $b \in B$. Como $a^{-1} \in A \subseteq C$ temos $b \in C, \log o b \in B \cap C$. Daí $c=a b \in A(B \cap C)$.

Vemos abaixo o exemplo essencial de reticulado modular: o reticulado $L_{R}(M)$ de $R$-submódulos de um $R$-módulo $M$, sendo $\vee$ e $\wedge$ a soma e a interseção de submódulos, respectivamente. 
Proposição 3.11. O reticulado $L_{R}(M)$ de $R$-submódulos de um $R$-módulo $M$ é modular, ou seja, para todos $A, B, C \in L_{R}(M)$ temos

$$
A \subseteq C \Longrightarrow A+(B \cap C)=(A+B) \cap C .
$$

Prova. Pela desigualdade modular, basta ver que $(A+B) \cap C \subseteq A+(B \cap C)$. Se $c \in(A+B) \cap C$ então $c=a+b$, com $a \in A$ e $b \in B$. Daí $b=c-a \in C$, uma vez que $A \subseteq C$. $\operatorname{logo} b \in B \cap C$, donde $c=a+b \in A+(B \cap C)$.

Teorema 3.12 (caracterização dos reticulados modulares). Um reticulado $\mathcal{L}$ é modular se, e somente se, para cada intervalo $\mathcal{I} \subseteq \mathcal{L}$, quaisquer dois elementos que são comparáveis e relacionados em $\mathcal{I}$ são iguais.

Prova. Pela definição (3.10), se $\mathcal{L}$ não é modular então existem $a, b, c \in \mathcal{L}$ tais que $a \prec c \mathrm{e}$

$$
a \vee(b \wedge c) \prec(a \vee b) \wedge c
$$

Definindo $a_{1}=a \vee(b \wedge c)$ e $c_{1}=(a \vee b) \wedge c$, podemos escrever $a \preccurlyeq a_{1} \prec c_{1} \preccurlyeq c$. Então

$$
b \wedge c_{1} \preccurlyeq b \wedge c \preccurlyeq a \vee(b \wedge c)=a_{1},
$$

donde $b \wedge c_{1} \preccurlyeq b \wedge a_{1}$ e portanto $b \wedge a_{1}=b \wedge c_{1}$ por monotonicidade; e

$$
a_{1} \vee b \succcurlyeq a \vee b \succcurlyeq(a \vee b) \wedge c=c_{1},
$$

donde $b \vee a_{1} \succcurlyeq b \vee c_{1}$ e portanto $b \vee a_{1}=b \vee c_{1}$ por monotonicidade. Defina

$$
b \wedge a_{1}=b \wedge c_{1}=a_{2} \quad \text { e } \quad b \vee a_{1}=b \vee c_{1}=c_{2} .
$$

Concluímos assim que $a_{1}, c_{1}$ são comparáveis e $b$-relacionados em $\left[a_{2}, c_{2}\right]$, mas não são iguais.

Reciprocamente, se $a^{\prime}, c^{\prime}$ são dois elementos distintos que são comparáveis e relacionados em $[a, c]$, digamos com

$$
a^{\prime} \wedge b=b \wedge c^{\prime}=a, \quad a^{\prime} \vee b=b \vee c^{\prime}=c \quad \text { e } \quad a \preccurlyeq a^{\prime} \prec c^{\prime} \preccurlyeq c,
$$

então $a^{\prime} \vee\left(b \wedge c^{\prime}\right)=a^{\prime} \prec c^{\prime}=\left(a^{\prime} \vee b\right) \wedge c^{\prime}$, e portanto $\mathcal{L}$ não é modular.

Um reticulado $\mathcal{L}$ pode ainda, eventualmente, satisfazer às condições

$$
\begin{aligned}
& (a \vee b) \wedge c=(a \wedge c) \vee(b \wedge c) \\
& (a \wedge b) \vee c=(a \vee c) \wedge(b \vee c)
\end{aligned}
$$

para todos $a, b, c \in \mathcal{L}$. Essas leis são chamadas leis distributivas. Nosso próximo passo é descrever reticulados que satisfazem a essas leis. 
Definição 3.13 (reticulados distributivos). Um reticulado para o qual valem as leis distributivas é dito um reticulado distributivo.

Proposição 3.14 (equivalência entre as leis distributivas). Para todo reticulado $\mathcal{L}$, são equivalentes:

(i) $(a \vee b) \wedge c=(a \wedge c) \vee(b \wedge c) \quad \forall a, b, c \in \mathcal{L}$;

(ii) $(a \wedge b) \vee c=(a \vee c) \wedge(b \vee c) \quad \forall a, b, c \in \mathcal{L}$;

(iii) $(a \vee b) \wedge c \preccurlyeq a \vee(b \wedge c) \quad \forall a, b, c \in \mathcal{L}$.

Em particular, todo reticulado distributivo é modular.

\section{Prova.}

$(i) \Rightarrow($ iii $)$ Como $a \wedge c \preccurlyeq a$, por $(3.5)$ temos $(a \wedge c) \vee(b \wedge c) \preccurlyeq a \vee(b \wedge c)$. Usando $(i)$, segue o resultado.

$($ iii $) \Rightarrow(i)$ Para $a, b, c \in \mathcal{L}$ quaisquer, temos $(a \vee b) \wedge c \preccurlyeq(b \wedge c) \vee a$; por monotonicidade e por (iii), temos

$$
\begin{aligned}
{[(a \vee b) \wedge c] \wedge c } & =(a \vee b) \wedge c \\
& \preccurlyeq[(b \wedge c) \vee a] \wedge c \\
& \preccurlyeq(b \wedge c) \vee(a \wedge c)
\end{aligned}
$$

Por outro lado, como $a \vee b \succcurlyeq(a \wedge c) \vee(b \wedge c)$ e $c \succcurlyeq(a \wedge c) \vee(b \wedge c)$ temos que $(a \vee b) \wedge c \succcurlyeq(a \wedge c) \vee(b \wedge c)$, o que prova a igualdade.

$(i) \Rightarrow(i i)$ Admitindo a validade da primeira lei distributiva, obtemos

$$
\begin{aligned}
(a \wedge b) \vee(a \wedge c) & =[a \vee(a \wedge c)] \wedge[b \vee(a \wedge c)] \\
& =a \wedge[(b \vee a) \wedge(b \vee c)] \\
& =[a \wedge(b \vee a)] \wedge(b \vee c) \\
& =a \wedge(b \vee c)
\end{aligned}
$$

para quaisquer $a, b, c \in \mathcal{L}$.

$(i i) \Rightarrow(i)$ Admitindo a validade da segunda lei distributiva, obtemos

$$
\begin{aligned}
(a \vee b) \wedge(a \vee c) & =[a \wedge(a \vee c)] \vee[b \wedge(a \vee c)] \\
& =a \vee[(b \wedge a) \vee(b \wedge c)] \\
& =[a \vee(b \wedge a)] \vee(b \wedge c) \\
& =a \vee(b \wedge c)
\end{aligned}
$$

para quaisquer $a, b, c \in \mathcal{L}$. 
Se $\mathcal{L}$ é um reticulado distributivo vale a condição (iii); logo, para todo intervalo $\mathcal{I}=[a, c] \subseteq \mathcal{L}$ vale o sinal de igualdade na desigualdade modular. Assim, todo reticulado distributivo é modular.

O teorema abaixo caracteriza os reticulados distributivos e é o principal resultado desta seção. Note que esse teorema torna ainda mais claro que todo reticulado distributivo é um reticulado modular, pois fortalece a hipótese do teorema (3.12).

Teorema 3.15 (caracterização dos reticulados distributivos). Um reticulado $\mathcal{L}$ é distributivo se, e somente se, para cada intervalo $\mathcal{I} \subseteq \mathcal{L}$, dois elementos relacionados em $\mathcal{I}$ são iguais.

\section{Prova.}

$\Leftrightarrow$ Suponha que $\mathcal{L}$ é distributivo e que $a, b$ são $c$-relacionados em um intervalo $\mathcal{I}=[u, v] \subseteq \mathcal{L}$, ou seja, tais que $a \wedge c=b \wedge c=u$ e $a \vee c=b \vee c=v$ Então

$$
a=a \wedge(b \vee c)=(a \wedge b) \vee(a \wedge c)=a \wedge b
$$

Isso mostra que $a \preccurlyeq b, \operatorname{logo} a=b$ pelo teorema (3.12).

$(\Leftarrow)$ Suponha que, para cada intervalo $\mathcal{I} \subseteq \mathcal{L}$, dois elementos relacionados em $\mathcal{I}$ são iguais. Em particular, dois elementos comparáveis e relacionados em um intervalo qualquer são iguais, $\operatorname{logo} \mathcal{L}$ é modular pela proposição (3.12).

Tome 3 elementos quaisquer $c_{1}, c_{2}, c_{3} \in \mathcal{L}$; defina

$$
\begin{array}{ll}
a_{1}=c_{2} \wedge c_{3} & b_{1}=c_{2} \vee c_{3} \\
a_{2}=c_{1} \wedge c_{3} & b_{2}=c_{1} \vee c_{3} \\
a_{3}=c_{1} \wedge c_{2} & b_{3}=c_{1} \vee c_{2}
\end{array}
$$

Como $a_{i} \preccurlyeq b_{i}$ para $i=1,2,3$, pela lei modular vem

$$
\left(a_{i} \vee c_{i}\right) \wedge b_{i}=a_{i} \vee\left(c_{i} \wedge b_{i}\right)=d_{i}
$$

Defina ainda

$$
u=a_{1} \vee a_{2} \vee a_{3} \quad \text { e } \quad v=b_{1} \wedge b_{2} \wedge b_{3} .
$$

Então, como $c_{2} \wedge b_{2} \preccurlyeq b_{1}$ e $c_{1} \preccurlyeq b_{2}$, aplicando a lei modular nos intervalos $\left[c_{2} \wedge b_{2}, b_{1}\right]$ e $\left[c_{1}, b_{2}\right]$ temos

$$
\begin{aligned}
\left(c_{2} \wedge b_{2}\right) \vee\left[c_{1} \wedge b_{1}\right] & =\left[\left(c_{2} \wedge b_{2}\right) \vee c_{1}\right] \wedge b_{1} \\
& =\left[c_{1} \vee\left(c_{2} \wedge b_{2}\right)\right] \wedge b_{1} \\
& =\left[\left(c_{1} \vee c_{2}\right) \wedge b_{2}\right] \wedge b_{1} \\
& =b_{3} \wedge b_{2} \wedge b_{1} \\
& =v .
\end{aligned}
$$


Assim,

$$
\begin{aligned}
d_{1} \vee d_{2} & =\left(c_{1} \wedge b_{1}\right) \vee a_{1} \vee\left(c_{2} \wedge b_{2}\right) \vee a_{2} \\
& =v \vee a_{1} \vee a_{2} \\
& =v
\end{aligned}
$$

Analogamente, encontramos $d_{1} \vee d_{2}=d_{1} \vee d_{3}=d_{2} \vee d_{3}=v$.

Como $a_{1} \preccurlyeq c_{2} \vee a_{2}$ e $a_{2} \preccurlyeq c_{1}$, aplicando a lei modular nos intervalos $\left[a_{1}, c_{2} \vee a_{2}\right]$ e $\left[a_{2}, c_{1}\right]$ temos

$$
\begin{aligned}
{\left[a_{1} \vee c_{1}\right] \wedge\left(c_{2} \vee a_{2}\right) } & =a_{1} \vee\left[c_{1} \wedge\left(c_{2} \vee a_{2}\right)\right] \\
& =a_{1} \vee\left[\left(a_{2} \vee c_{2}\right) \wedge c_{1}\right] \\
& =a_{1} \vee\left[a_{2} \vee\left(c_{2} \wedge c_{1}\right)\right] \\
& =a_{1} \vee a_{2} \vee a_{3} \\
& =u .
\end{aligned}
$$

Assim,

$$
\begin{aligned}
d_{1} \wedge d_{2} & =\left(a_{1} \vee c_{1}\right) \wedge b_{1} \wedge\left(a_{2} \vee c_{2}\right) \wedge b_{2} \\
& =u \wedge b_{1} \wedge b_{2} \\
& =u
\end{aligned}
$$

Analogamente, encontramos $d_{1} \wedge d_{2}=d_{1} \wedge d_{3}=d_{2} \wedge d_{3}=u$. Verificamos, portanto, que $d_{i}$ e $d_{j}$ são relacionados no intervalo $[u, v]$ sempre que $i \neq j$. Por hipótese, temos $d_{1}=d_{2}=d_{3}$, logo $u=d_{1}=d_{2}=d_{3}=v$. Assim,

$$
\begin{gathered}
c_{1} \vee a_{1} \succcurlyeq u=v \succcurlyeq c_{2} \wedge b_{2}, \\
c_{1} \vee\left(c_{3} \wedge c_{2}\right) \succcurlyeq\left(c_{1} \vee c_{3}\right) \wedge c_{2} .
\end{gathered}
$$

Como $c_{1}, c_{2}, c_{3} \in \mathcal{L}$ foram escolhidos de forma arbitrária, da proposição (3.14) segue que $\mathcal{L}$ é distributivo.

Corolário 3.16 (lei do cancelamento). Um reticulado $\mathcal{L}$ é distributivo se, e somente se, vale a lei do cancelamento, ou seja, se

$$
a \wedge b=a \wedge c \quad e \quad a \vee b=a \vee c \Longrightarrow b=c
$$

para todos $a, b, c \in \mathcal{L}$.

Prova. Afirmar a lei do cancelamento implica em dizer que, para qualquer intervalo $\mathcal{I} \subseteq \mathcal{L}$, quaisquer dois elementos relacionados em $\mathcal{I}$ são iguais, ou seja, que $\mathcal{L}$ é distributivo (3.15). Reciprocamente, seja $\mathcal{L}$ um reticulado distributivo. Defina $u, v \in \mathcal{L}$ pondo $u=a \wedge b$ e $v=a \vee b$; por hipótese, $b$ e $c$ são $a$-relacionados em $[u, v]$. Do teorema (3.15) segue que $b=c$. 


\subsection{Teorema de Behrens}

Nesta seção trataremos de reticulados de submódulos e de ideais (geralmente, à esquerda). Nosso primeiro objetivo é descrever o reticulado $L_{R}(M)$ de submódulos de um módulo distributivo $M$ sobre um anel $R$ satisfazendo uma série de hipóteses. No final da seção, o teorema de Behrens (3.30) descreve o reticulado de ideais à esquerda de $R$ no caso em que $R$ é um anel semiperfeito, noetheriano à esquerda e com uma condição de finitude sobre o radical $J(R)$.

A próxima seção 3.3 trata do teorema de Stephenson, que generaliza substancialmente o teorema de Behrens. Deste modo, essas seções ilustram o progresso da pesquisa na área da década de 1960 à década de 1970. No entanto, o leitor apressado, ávido por resultados mais gerais, pode seguir diretamente para a seção 3.3 .

A exposição segue o livro de Behrens [BE]. Para começar, fixamos mais uma vez a terminologia utilizada.

Definição 3.17 (anéis e módulos distributivos). Sejam $R$ um anel e $M$ um $R$-módulo. $M$ é dito distributivo se o reticulado $L_{R}(M)$ é distributivo. $O$ anel $R$ é dito distributivo (à esquerda) se o reticulado $L_{R}\left({ }_{R} R\right)$ é distributivo. Também dizemos, de forma mais sintética, que $M$ e $R$ são um $\boldsymbol{D}$-módulo $e$ um D-anel (à esquerda).

Exemplo 3.18. O módulo $\mathbb{Z} \mathbb{Z}$ é distributivo.

Isso ocorre pois $[m,(n, p)]=([m, n],[m, p])$ para quaisquer inteiros positivos $m, n, p$, donde

$$
m \mathbb{Z} \cap(n \mathbb{Z}+p \mathbb{Z})=(m \mathbb{Z} \cap n \mathbb{Z})+(m \mathbb{Z} \cap p \mathbb{Z})
$$

Exemplo 3.19. Seja $M$ um $R$-módulo. Se $M$ possui subconjuntos linearmente independentes sobre $R$ com mais do que um elemento, então $M$ não é distributivo.

Com efeito, considere dois elementos linearmente independentes $a, b \in M$. Defina $A=R a, B=R b$ e $C=R(a+b)$; é fácil ver que

$$
C=(A+B) \cap C \neq A \cap C+B \cap C=0+0=0
$$

Proposição 3.20. Um produto direto de D-anéis à esquerda é um D-anel à esquerda. 
Prova. Seja $R$ um anel tal que $R=\prod_{i \in I} R_{i}$, sendo $R_{i}$ um D-anel à esquerda para cada $i \in I$, e considere os ideais à esquerda $A, B, C \in L_{R}\left({ }_{R} R\right)$. É claro que podemos escrever $A=\prod_{i \in I} A_{i}$, sendo $A_{i}$ um ideal à esquerda de $R_{i}$ para cada $i \in I$, e similarmente para $B$ e $C$. É claro, também, que valem as implicações

$$
\begin{array}{ll}
A \cap B=A \cap C \quad \Longrightarrow A_{i} \cap B_{i}=A_{i} \cap C_{i} & \forall i \in I ; \\
A+B=A+C \Longrightarrow A_{i}+B_{i}=A_{i}+C_{i} & \forall i \in I .
\end{array}
$$

Pela lei do cancelamento (3.16), temos $B_{i}=C_{i}$ para todo $i \in I$, donde $B=C$. Como $A, B, C$ são arbitrários, pela lei do cancelamento temos que $R$ é um D-anel à esquerda.

Proposição 3.21. Quocientes e submódulos de módulos distributivos são distributivos.

Prova. Sejam $R$ um anel, ${ }_{R} M$ um módulo distributivo e $N$ um $R$-submódulo de $M$. Se $A, B, C \in L_{R}(N)$ então $A, B, C \in L_{R}(M)$, então é claro que $L_{R}(N)$ é distributivo. Pelo teorema da correspondência para módulos, a projeção natural $M \rightarrow M / N$ induz um isomorfismo de reticulados entre $L_{R}(M / N)$ e o subreticulado de $L_{R}(M)$ consistindo de submódulos de $M$ contendo $N$. Logo $L_{R}(M / N)$ é distributivo.

A proposição a seguir nos mostra uma primeira característica essencial de módulos distributivos: seus submódulos minimais não são isomorfos. Esse fato será generalizado no corolário (3.34).

Proposição 3.22. Seja $R$ um anel e $U \neq V$ dois submódulos minimais do R-módulo M. Se $M$ é distributivo então U e V não são isomorfos.

Prova. Suponha, por absurdo, que $\varphi: U \rightarrow V$ é um $R$-isomorfismo. Então temos que $W=\{u+\varphi(u): u \in U\}$ é um $R$-submódulo de $M$ e, como $W \subseteq U+V$ e $M$ é distributivo, temos

$$
0 \neq W=W \cap(U+V)=(W \cap U)+(W \cap V) .
$$

Se mostrarmos que $W \cap U=W \cap V=0$ teremos uma contradição. De fato, se $x \in W \cap U$ então $x=u+\varphi(u)=u^{\prime}$ para alguns $u, u^{\prime} \in U$. Como $U \cap V=0$ por minimalidade, temos $x-u=\varphi(u)=0$, donde $x=0$. Analogamente se mostra que $W \cap V=0$.

Corolário 3.23. Sejam $R$ um anel artiniano simples e $M$ um $R$-módulo não-nulo. O reticulado $L_{R}(M)$ é distributivo se e somente se $M$ é simples. 
Prova. Se $M$ é simples, $L_{R}(M)$ é trivialmente distributivo. Reciprocamente, admita que $L_{R}(M)$ é distributivo. Sabemos que todo anel artiniano simples é isomorfo ao anel semisimples $\mathbb{M}_{n}(D)$ para algum anel de divisão $D$ pelo teorema de Wedderburn (1.29), e que todo módulo não-nulo $M$ sobre um anel semisimples é uma soma direta de submódulos simples (1.36). Suponha que $M$ não é simples e que $L_{R}(M)$ é distributivo. Então $M$ tem pelo menos dois submódulos simples distintos, e pela proposição anterior eles não são isomorfos; mas isso é uma contradição ao fato de que todos os módulos simples sobre um anel artiniano simples são isomorfos entre si.

$O$ resultado acima, de caráter bastante restrito, pode ser generalizado para o caso em que $R / J(R)$ é um anel artiniano simples. Mesmo assim, precisaremos de hipóteses adicionais fortes.

Proposição 3.24. Sejam $R$ um anel e $J=J(R)$ seu radical. Suponha que $R / J$ é um anel artiniano simples e que ${ }_{R} M$ é um módulo noetheriano tal que $\bigcap_{s=1}^{\infty} J^{s} M=0$. Então, $M$ é distributivo se e somente se $L_{R}(M)$ consiste apenas de termos da série de Loewy

$$
M \supseteq J M \supseteq J^{2} M \supseteq \cdots
$$

mầs o submódulo nulo. Nesse caso, todo R-submódulo de $M$ é cíclico.

Prova. Suponha, a princípio, que $L_{R}(M)=\left\{M, J M, J^{2} M, \ldots, 0\right\}$. É fácil ver que $L_{R}(M)$ é um reticulado distributivo.

Reciprocamente, se $M$ é distributivo então é fácil ver que a distributividade de $L_{R}(M)$ implica na distributividade do subreticulado consistindo de $R$-submódulos $U$ tais que $J^{s+1} M \subseteq U \subseteq J^{s} M$. Logo, pelo teorema da correspondência, o reticulado de $Q_{s}=J^{s} M / J^{s+1} M$ é distributivo. Como $J \subseteq A n n_{R} Q_{s}$, da proposição (1.7) segue que $Q_{s}$ é um $\bar{R}$-módulo sobre o anel artiniano simples $\bar{R}=R / J$; portanto, se $Q_{s} \neq 0$ então $Q_{s}$ é simples, pelo corolário (3.23).

Seja $U \neq 0$ um submódulo de $M$. Como $\bigcap_{s} J^{s} M=0$ por hipótese, existe um expoente máximo $t \geqslant 0$ tal que $U \subseteq J^{t} M$; vejamos que $U=J^{t} M$. Como $Q_{t}=J^{t} M / J^{t+1} M$ é simples, não existe um $R$-submódulo de $M$ estritamente entre $J^{t+1} M$ e $J^{t} M$. Logo, podemos escrever

$$
J^{t} M=U+J^{t+1} M .
$$

Como $M$ é noetheriano por hipótese, pelo teorema (1.12) $J^{t} M$ é finitamente gerado; pelo lema de Nakayama (1.42) segue que $U=J^{t} M$.

Resta mostrar que todo $R$-submódulo de $M$ é cíclico. Se $J^{s} M \neq 0$, tome $m_{s} \in J^{s} M \backslash J^{s+1} M$. Então $R m_{s}$ é um $R$-submódulo de $J^{s} M$ e $R m_{s} \not \subset J^{s+1} M$; como $J^{s} M / J^{s+1} M$ é simples, temos $J^{s} M=R m_{s}$. 
Podemos substituir a hipótese de $R / J$ ser artiniano simples pela hipótese mais fraca de que $R$ seja um anel semiperfeito; nesse caso, ainda é possível descrever a estrutura de $L_{R}(M)$, se soubermos de antemão que esse reticulado é distributivo. Esse é o objetivo do próximo teorema (3.26).

Lema 3.25. Seja $R$ um anel, $e \in R$ um idempotente e $M$ um $R$-módulo. Então a aplicação $\phi: U \mapsto e U$, definida para todo submódulo $U \in L_{R}(M)$, é um epimorfismo de $L_{R}(M)$ sobre $L_{\text {eRe }}(e M)$. Em particular, se $L_{R}(M)$ é distributivo então $L_{e R e}(e M)$ também é.

Prova. Sejam $U, V \in L_{R}(M)$. Temos $e(U+V)=e U+e V, U \cap e M=e U$, donde segue também $e(U \cap V)=e U \cap e V$. Portanto, $\phi$ é um homomorfismo de reticulados. Para ver que $\phi$ é epimorfismo, tome $P=e P \in L_{e}$ e $(e M)$; então $R P \in L_{R}(M)$ e $\phi(R P)=e R P=e R e P=P$.

\section{Notação}

Seja $R$ um anel, $J$ seu radical e $M$ um $R$-módulo tal que $\bigcap_{n=1}^{\infty} J^{n} M=0$. Denotaremos por $J^{0} M$ o módulo $M$ e por $J^{\infty} M$ o submódulo nulo. Dessa forma, a notação $J^{n} M$ faz sentido para qualquer $n \in\{0\} \cup \mathbb{N} \cup\{\infty\}$.

Lembramos ainda que, se $R$ é um anel semiperfeito e $J=J(R)$, as unidades dos somandos diretos simples de $R / J$ podem ser levantadas a uma família completa de idempotentes ortogonais $\left\{e_{1}, \ldots, e_{q}\right\}$ em $R$. Definindo $R_{i}=e_{i} e_{i}$ para $i=1, \ldots, q$, temos que $J_{i}=J\left(R_{i}\right)=e_{i} J e_{i}$ para cada $i$ (proposição 2.19).

Teorema 3.26. Seja $R$ um anel semiperfeito com radical $J=J(R)$.

Hipóteses:

- ${ }_{R} M$ é um módulo noetheriano tal que $\bigcap_{n=1}^{\infty} J^{n} M=0$.

- $L_{R}(M)$ é distributivo.

Conclusões:

- Todo $R$-submódulo $U \subseteq M$ pode ser escrito de modo único como soma direta (de grupos abelianos) $U=e_{1} U \oplus \cdots \oplus e_{q} U$ de $R_{i}$-submódulos $d a$ forma $e_{i} U=J_{i}^{s_{i}} M$, onde $s_{i} \in\{0\} \cup \mathbb{N} \cup\{\infty\}$.

- $L_{R}(M)$ é isomorfo a um certo subreticulado do reticulado consistindo de q-tuplas $\left(s_{1}, \ldots, s_{q}\right), s_{i} \in\{0\} \cup \mathbb{N} \cup\{\infty\}$, ordenado parcialmente por $\left(s_{1}, \ldots, s_{q}\right) \preccurlyeq\left(t_{1}, \ldots, t_{q}\right) \Longleftrightarrow s_{i} \geqslant t_{i}, i=1, \ldots, q$.

- Todo R-submódulo de $M$ é cíclico. 
Prova. Por hipótese, temos que

$$
\frac{R_{i}}{J_{i}}=\frac{R_{i}}{J \cap R_{i}} \simeq \frac{R_{i}+J}{J}=\bar{e}_{i} \bar{R}_{\bar{e}_{i}}
$$

é uma componente simples do anel artiniano semisimples $\bar{R}$ (teorema 2.22). Logo, para todo $R$-submódulo $U$ do módulo distributivo noetheriano ${ }_{R} M$ podemos escrever

$$
U=1 U=e_{1} U \oplus \cdots \oplus e_{q} U=U_{1} \oplus \cdots \oplus U_{q}
$$

ou seja, $U$ é uma soma direta (como grupos abelianos) dos $R_{i}$-submódulos $U_{i}=e_{i} U$ dos $R_{i}$-módulos $M_{i}=e_{i} M$.

$\bar{R}_{i}$ é artiniano simples; como $J_{i}^{s} M_{i}=\left(e_{i} J e_{i}\right)^{s} M$ está contido em $J^{s} M$, então $\bigcap_{s=1}^{\infty} J_{i}^{s} M_{i}=0$. O reticulado $L_{R}(M)$ é distributivo, logo $L_{R_{i}}\left(M_{i}\right)$ é distributivo (lema 3.25). Assim, valem as hipóteses da proposição (3.24) para o $R_{i}$-módulo noetheriano $M_{i}$, donde deduzimos que o reticulado $L_{R_{i}}\left(M_{i}\right)$ consiste da série de Loewy

$$
M_{i} \supseteq J_{i} M_{i} \supseteq J_{i}^{2} M_{i} \supseteq \cdots
$$

mais o submódulo nulo de $M_{i}$. Por outro lado, pela equação (3.1), para cada $U \in L_{R}(M)$ podemos escrever $U=e_{1} U \oplus \cdots \oplus e_{q} U$. Portanto, como $e_{i} U=J_{i}^{s_{i}} M_{i}=J_{i}^{s_{i}} M$ para algum $s_{i} \in\{0\} \cup \mathbb{N} \cup\{\infty\}$, cada submódulo $U \subseteq M$ pode ser escrito, de modo único, na forma

$$
U=J_{1}^{s_{1}} M \oplus \cdots \oplus J_{q}^{s_{q}} M
$$

uma soma direta dos grupos abelianos $J_{i}^{s_{i}} M$.

Denote por $\left(s_{1}, \ldots, s_{q}\right)$ a $q$-tupla correspondente a $U$ e por $\left(t_{1}, \ldots, t_{q}\right)$ a $q$-tupla correspondente a um $R$-submódulo $V$ na decomposição acima. Daí $\left(\min \left\{s_{i}, t_{i}\right\}: 1 \leqslant i \leqslant q\right)$ e $\left(\max \left\{s_{i}, t_{i}\right\}: 1 \leqslant i \leqslant q\right)$ são as $q$-tuplas que correspondem a $U+V$ e $U \cap V$, respectivamente. Note que nem toda $q-$ tupla $\left(n_{1}, \ldots, n_{q}\right)$ de elementos de $\{0\} \cup \mathbb{N} \cup\{\infty\}$ ocorre como uma $q$-tupla de expoentes correspondendo a algum $R$-submódulo de $M$ pois, em geral, $\bigoplus_{i=1}^{q} J_{i}^{s_{i}} M$ é um subgrupo de $M$, não necessariamente um submódulo, como veremos no exemplo (3.27) a seguir.

Finalmente, vejamos que todo $R$-submódulo $U \subseteq M$ é cíclico. Escreva $U$ como na equação (3.1); pela proposição (3.24) temos que cada $R_{i}$-submódulo $e_{i} U \subseteq e_{i} M$ é cíclico, digamos $e_{i} U=R_{i} u_{i}$ para algum $u_{i} \in e_{i} U$, para cada $i \in\{1, \ldots, q\}$. Defina $u=u_{1}+\cdots+u_{q}$. Então $R_{i} u_{i}=R_{i} e_{i} u$ para todo $i \in\{1, \ldots, q\}$. Logo $U=\sum_{i=1}^{q} R_{i} u_{i} \subseteq R u \subseteq U$, donde $U=R u$. 
Exemplo 3.27. Sejam $R$ e $M$ como no teorema (3.26). Nem toda q-tupla $\left(n_{1}, \ldots, n_{q}\right)$ de elementos de $\{0\} \cup \mathbb{N} \cup\{\infty\}$ ocorre como uma q-tupla de expoentes correspondendo a algum $R$-submódulo de $M$.

Usando a notação do teorema acima, definimos $R$ e $M$ por

$$
R=\left[\begin{array}{lll}
F & F & F \\
0 & F & F \\
0 & 0 & F
\end{array}\right] \quad \text { e } \quad M=\left[\begin{array}{c}
F \\
F \\
F
\end{array}\right]
$$

sendo $F$ um corpo não-nulo qualquer. Note que $R$ e $M$ satisfazem às hipóteses do teorema (3.26), $\operatorname{com} q=3 \mathrm{e}$

$$
J=\left[\begin{array}{ccc}
0 & F & F \\
0 & 0 & F \\
0 & 0 & 0
\end{array}\right], e_{1}=\left[\begin{array}{lll}
1 & 0 & 0 \\
0 & 0 & 0 \\
0 & 0 & 0
\end{array}\right], e_{2}=\left[\begin{array}{lll}
0 & 0 & 0 \\
0 & 1 & 0 \\
0 & 0 & 0
\end{array}\right], e_{3}=\left[\begin{array}{lll}
0 & 0 & 0 \\
0 & 0 & 0 \\
0 & 0 & 1
\end{array}\right] \text {. }
$$

Observe que à tripla $(0,1,0)$ corresponde o subgrupo

$$
\left(e_{1} J e_{1}\right)^{0} M \oplus\left(e_{2} J e_{2}\right)^{1} M \oplus\left(e_{3} J e_{3}\right)^{0} M=\left[\begin{array}{c}
F \\
0 \\
F
\end{array}\right] \subset M,
$$

que não é um $R$-submódulo de $M$. Observe ainda que $L_{R}(M)$ é isomorfo ao reticulado $(1,1,1) \preccurlyeq(0,1,1) \preccurlyeq(0,0,1) \preccurlyeq(0,0,0)$.

Podemos especializar o teorema (3.26) acima para o caso particular em que o módulo distributivo ${ }_{R} M$ em questão é ${ }_{R} R$. Esse é o conteúdo do teorema de Behrens (3.30).

Definição 3.28 (anéis uniseriais). Um anel $R$ é dito um anel uniserial à esquerda (à direita) se seus ideais à esquerda (à direita) formam uma cadeia totalmente ordenada.

Um anel uniserial noetheriano comutativo não-artiniano é apresentado no exemplo (2.63) à página 47 .

Definição 3.29 (expoente de um ideal bilateral). Seja $J \subseteq R$ um ideal bilateral de um anel $R$ tal que $\bigcap_{n=1}^{\infty} J^{n}=0$, e considere a cadeia descendente $J \supseteq J^{2} \supseteq \cdots$ Definimos por $\exp (J)$ o indice de nilpotência de $J$ se $J$ é nilpotente, e dizemos que $\exp (J)=\infty$ se $J$ não é nilpotente. $\exp (J)$ é chamado de expoente de $J$.

Teorema 3.30 (Behrens). Seja $R$ um anel semiperfeito e noetheriano à esquerda, com radical $J=J(R)$ tal que $\bigcap_{n=1}^{\infty} J^{n}=0$. Então o reticulado $L_{R}(R)$ de ideais à esquerda de $R$ é distributivo se e somente se $R$ é soma direta (de anéis) de um número finito de anéis locais uniseriais à esquerda. 
Prova. Usaremos a mesma notação do teorema (3.26). Suponha que $L_{R}(R)$ é distributivo; então $L_{R_{i}}\left(e_{i} R\right)$ é distributivo (lema 3.25). Como cada $R_{i} / J_{i}$, $i=1, \ldots, q$, é simples, do teorema (3.24) segue que os $R_{i}$-submódulos do $R_{i}$-módulo $e_{i} R$ são apenas os membros da série de Loewy $J_{i}^{n} R, n=0,1, \ldots$ Em particular, $J_{i} R$ é o submódulo maximal de $e_{i} R$, portanto $e_{i} R / J_{i} R$ é um $R_{i}$-módulo simples. Logo $e_{i} R / J_{i} R$ é cíclico, gerado pelo elemento não-nulo $e_{i}+J_{i} R$ (proposição 1.2). Então, como $R_{i}=e_{i} R e_{i}$, temos

$$
e_{i} R=R_{i}+J_{i} R
$$

Por um lado, temos

$$
e_{i} R=e_{i} R e_{i}+\left(\sum_{j \neq i} e_{i} R e_{j}\right)
$$

e, por outro lado, temos

$$
R_{i}+J_{i} R=e_{i} R e_{i}+\left(\sum_{j \neq i} J_{i} R e_{j}\right),
$$

onde os segundos membros de (3.3) e (3.4) são somas diretas de grupos abelianos. Portanto, pela equação (3.2) temos

$$
e_{i} R e_{j}=J_{i} R e_{j} \quad \operatorname{para} j \neq i,
$$

e assim

$$
e_{i} R e_{j}=J_{i} R e_{j}=J_{i}^{2} R e_{j}=J_{i}^{3} R e_{j}=\cdots=J_{i}^{n} R e_{j}=\cdots ;
$$

como $\bigcap_{n=1}^{\infty} J^{n}=0$, temos $e_{i} R e_{j}=0$ se $i \neq j$. Isso implica que

$$
R=R_{1} \oplus \cdots \oplus R_{q}
$$

e que portanto $R$ é uma soma direta (como anéis) dos anéis locais $R_{i}$.

A série de Loewy do $R_{i}$-módulo $e_{i} R$ consiste dos $J_{i}^{n} R=J_{i}^{n}, n=0,1, \ldots$ Esses $R_{i}$-módulos são, portanto, ideais bilaterais do anel $R_{i}$. Vimos que $R=R_{1} \oplus \cdots \oplus R_{q}, \log$ o esses ideais são também ideais bilaterais de $R$. Pelo teorema (3.26), todo ideal à esquerda $L \subseteq R$ pode ser escrito, de modo único, como uma soma direta

$$
e_{1} L \oplus \cdots \oplus e_{q} L=J_{1}^{n_{1}} \oplus \cdots \oplus J_{q}^{n_{q}}
$$

dos ideais bilaterais $J_{i}^{n_{i}}$ de $R$. Observe que, assim, ao contrário do que acontece no teorema (3.26), toda q-tupla $\left(n_{1}, \ldots, n_{q}\right)$ ocorre como q-tupla de expoentes de algum ideal à esquerda de $R$. 
Reciprocamente, se $R$ é uma soma direta de um número finito de anéis uniseriais à esquerda locais $R_{i}, i=1, \ldots, q$, então é claro que todo ideal à esquerda de $R$ é soma direta de potências dos radicais $J\left(R_{i}\right)$, ou seja, todo ideal à esquerda de $R$ pode ser escrito na forma $J_{1}^{n_{1}} \oplus \cdots \oplus J_{q}^{n_{q}}$. Portanto, o reticulado $L_{R}(R)$ consiste de $q$-tuplas $\left(s_{1}, \ldots, s_{q}\right), s_{i} \in\left\{0,1, \ldots, \exp \left(J_{i}\right)\right\}$, ordenado parcialmente por

$$
\left(s_{1}, \ldots, s_{q}\right) \preccurlyeq\left(t_{1}, \ldots, t_{q}\right) \Longleftrightarrow s_{i} \geqslant t_{i}, \quad i=1, \ldots, q .
$$

Esse reticulado é distributivo.

\subsection{Teorema de Stephenson}

$\mathrm{Na}$ seção anterior, caracterizamos módulos distributivos noetherianos sobre anéis semiperfeitos com uma condição de finitude adicional sobre o radical (teorema de Behrens). Esses resultados foram generalizados de uma forma substancial por Stephenson na década de 1970. Nesta seção, diversos fatos referentes a módulos distributivos e seus homomorfismos são apresentados. O principal resultado é o teorema de Stephenson (3.46), que caracteriza anéis distributivos à esquerda em termos de certas condições de finitude.

A exposição segue os artigos de Stephenson [ST I] e [ST II].

Os próximos dois lemas preparam a proposição (3.33), que oferece uma caracterização um pouco mais geral de D-módulos.

Lema 3.31. Sejam $M$ um R-módulo e $A, B \in L_{R}(M)$ tais que $A \cap B=0$. Então há uma correspondência biunívoca entre as projeções $p: A \oplus B \rightarrow B$ e o conjunto dos complementos diretos de $B$ em $A \oplus B$, denotado por

$$
L(A, B)=\left\{X \in L_{R}(M): X \oplus B=A \oplus B\right\} .
$$

Essa correspondência é definida por $p \longleftrightarrow \operatorname{ker} p$.

Prova. Tome uma projeção $p: A \oplus B \rightarrow B$; para provar que ker $p \in L(A, B)$ precisamos mostrar que $\operatorname{ker} p \oplus B=A \oplus B$. Tome $a+b \in A \oplus B$; então $p(a+b)=b^{\prime} \in B$. Como $p$ induz a identidade em $B$ temos $p\left(a+b-b^{\prime}\right)=0$, donde $a+b-b^{\prime} \in \operatorname{ker} p$. Logo $a+b \in \operatorname{ker} p \oplus B$, donde $A \oplus B \subseteq \operatorname{ker} p \oplus B$. A inclusão contrária é óbvia.

Inversamente, resta mostrar que a cada $C \in L(A, B)$ corresponde uma única projeção $p: A \oplus B \rightarrow B$ tal que ker $p=C$. Como $C \oplus B=A \oplus B$, para todos $a \in A, b \in B$ temos $a+b=c+b^{\prime}$ para certos $c \in C, b^{\prime} \in B$ unicamente determinados. Assim, é fácil ver que $c+b^{\prime} \mapsto b^{\prime}$ define uma projeção $p$ tal 
que ker $p=C$. Se há outra projeção $\pi: A \oplus B \rightarrow B$ tal que $\operatorname{ker} \pi=C$, então $\pi(a+b)=\pi\left(c+b^{\prime}\right)=\pi(c)+\pi\left(b^{\prime}\right)=b^{\prime}=p(a+b)$, logo $p$ é único.

\section{Notação}

Dado um submódulo $P$ de um $R$-módulo $M$ e um homomorfismo $\alpha: P \rightarrow M$ tal que $P \cap \alpha(P)=0$, definimos $\bar{P}(\alpha)=(1-\alpha)(P)$, onde 1 é a inclusão de $P$ em $M$.

Dessa forma, $\bar{P}(\alpha)$ é um complemento direto de $\alpha(P)$ em $P \oplus \alpha(P)$, logo $\bar{P}$ define uma aplicação de $\operatorname{Hom}_{R}(P, M)$ em $L(P, \alpha(P))$. No próximo lema vemos uma situação onde essa aplicação é bijetora.

Lema 3.32. Sejam $A, B \in L_{R}(M)$ tais que $A \cap B=0$ e $\alpha \in \operatorname{Hom}_{R}(A, B)$. Então $\bar{A}$ define uma bijeção entre $\operatorname{Hom}_{R}(A, B)$ e $L(A, B)$.

Prova. Dado $\alpha \in \operatorname{Hom}_{R}(A, B)$, seja $\alpha^{*} \in \operatorname{Hom}_{R}(A \oplus B, B)$ o homomorfismo (único) induzindo $\alpha$ em $A$ e a identidade em $B$. É claro que $\alpha^{*}$ é uma projeção de $A \oplus B$ sobre $B$. Observe então que ker $\alpha^{*}=(1-\alpha)(A)=\bar{A}(\alpha)$; portanto, pelo lema (3.31), temos que $\bar{A}(\alpha) \in L(A, B)$ corresponde univocamente a $\alpha \in \operatorname{Hom}_{R}(A, B)$ pela aplicação $\bar{A}$.

Reciprocamente, tome $C \in L(A, B)$. Pelo lema (3.31), a $C$ corresponde uma única projeção $p: A \oplus B \rightarrow B$ tal que $C=\operatorname{ker} p$. É claro que $p$ é da forma $\alpha^{*}$ para um único homomorfismo $\alpha \in \operatorname{Hom}_{R}(A, B)$, logo

$$
C=\operatorname{ker} p=\operatorname{ker} \alpha^{*}=\bar{A}(\alpha) .
$$

Proposição 3.33. Seja $R$ um anel e $M$ um $R$-módulo. Então $M$ é um $D$-módulo se e somente se

$$
\operatorname{Hom}_{R}\left(\frac{A}{A \cap B}, \frac{B}{A \cap B}\right)=0
$$

para todos $A, B \in L_{R}(M)$.

Prova. Pelo lema (3.32), se $P \cap Q=0$ então existe uma bijeção entre $\operatorname{Hom}_{R}(P, Q)$ e o conjunto $L(P, Q)$ de complementos diretos de $Q$ em $P \oplus Q$. Tome $P=A /(A \cap B)$ e $Q=B /(A \cap B)$; então há uma bijeção entre os complementos de $B /(A \cap B)$ em $(A+B) /(A \cap B)$ e os homomorfismos em $\operatorname{Hom}_{R}(A /(A \cap B), B /(A \cap B))$. Isso é o mesmo que dizer que existe uma bijeção entre $\operatorname{Hom}_{R}(A /(A \cap B), B /(A \cap B))$ e os complementos de $B$ em relação ao intervalo $[A \cap B, A+B]$ do reticulado $L_{R}(M)$.

Como $M$ é distributivo, quaisquer dois complementos relativos em $L_{R}(M)$ são iguais, pelo teorema (3.15); $\operatorname{logo}_{0} \operatorname{Hom}_{R}(A /(A \cap B), B /(A \cap B))$ tem apenas um elemento, que deve ser portanto o homomorfismo nulo. 
Corolário 3.34. Sejam $A, B R$-submódulos do R-módulo distributivo $M$.

(i) $S e A+B=M$, então $\operatorname{Hom}_{R}(M / A, M / B)=0$.

(ii) Se $A \cap B=0$, então $\operatorname{Hom}_{R}(A, B)=0$.

\section{Prova.}

(i) Se $A+B=M$ então $M / A \simeq B /(A \cap B)$ e $M / B \simeq A /(A \cap B)$, pelo segundo teorema do isomorfismo.

(ii) Isso é claro.

Observe que o corolário acima generaliza a proposição (3.22).

Corolário 3.35. Todo idempotente de um D-anel é central.

Prova. Se $R$ é um D-anel e $e \in R$ é idempotente, então $R e \cap R(1-e)=0$; de fato, se $a \in R e \cap R(1-e)$ então $a=r e=s-$ se para alguns $r, s \in R$, donde $a=r e=r e^{2}=s e-s e^{2}=0$. Pelo corolário (3.34) temos, portanto,

$$
\operatorname{Hom}_{R}(R e, R(1-e))=0 \quad \text { e } \quad \operatorname{Hom}_{R}(R(1-e), R e)=0 .
$$

Do lema (2.5) segue que $e R(1-e)=(1-e) R e=0$. Para todo $r \in R$ temos então que $\operatorname{er}(1-e)=(1-e) r e=0$, ou seja, re $=e r=$ ere. Então $e$ é central.

Lema 3.36. Sejam $R$ um anel, $P, M, Q R$-módulos, $\varphi \in \operatorname{Hom}_{R}(P, M) e$ $\psi \in \operatorname{Hom}_{R}(M, Q)$. Se $A, B \in L_{R}(M)$ então

(i) $\varphi^{-1} \varphi \varphi^{-1}(A)=\varphi^{-1}(A)$.

(ii) $\varphi \varphi^{-1}(A)=A \cap \operatorname{im} \varphi$.

(iii) $A \subseteq \operatorname{im} \varphi$ ou $B \subseteq \operatorname{im} \varphi \Longrightarrow \varphi^{-1}(A)+\varphi^{-1}(B)=\varphi^{-1}(A+B)$.

(iv) $\psi \psi^{-1} \psi(A)=\psi(A)$.

(v) $\psi^{-1} \psi(A)=A+\operatorname{ker} \psi$.

(vi) $A \supseteq \operatorname{ker} \psi$ ou $B \supseteq \operatorname{ker} \psi \Longrightarrow \psi(A) \cap \psi(B)=\psi(A \cap B)$.

Prova. Para o item (ii), observe que

$$
a \in A \cap \operatorname{im} \varphi \Longleftrightarrow \exists a^{\prime} \in \varphi^{-1}(A): \varphi\left(a^{\prime}\right)=a \Longleftrightarrow a \in \varphi \varphi^{-1}(A) .
$$

Temos que $\varphi^{-1}(A \cap \operatorname{im} \varphi)=\varphi^{-1}(A)$, pois

$$
x \in \varphi^{-1}(A) \Longleftrightarrow \varphi(x) \in A \cap \operatorname{im} \varphi \Longleftrightarrow x \in \varphi^{-1}(A \cap \operatorname{im} \varphi) ;
$$


$\operatorname{logo} \varphi^{-1}\left[\varphi \varphi^{-1}(A)\right]=\varphi^{-1}(A \cap \operatorname{im} \varphi)=\varphi^{-1}(A)$, provando $(i)$. Note que $x \in \varphi^{-1}(A)+\varphi^{-1}(B) \Longrightarrow \exists a, b \in A, B: \varphi(x)=a+b \Longleftrightarrow x \in \varphi^{-1}(A+B)$, $\operatorname{logo} \varphi^{-1}(A)+\varphi^{-1}(B) \subseteq \varphi^{-1}(A+B)$. Reciprocamente, se $x \in \varphi^{-1}(A+B)$ e, digamos, $A \subseteq \operatorname{im} \varphi$, então $\varphi(x)=a+b$ para alguns $a \in A, b \in B$ e existe $a^{\prime} \in \varphi^{-1}(A)$ tal que $\varphi\left(a^{\prime}\right)=a$. Assim, temos

$$
\varphi(x)=a+b \Longleftrightarrow \varphi\left(x-a^{\prime}\right)=b \Rightarrow x-a^{\prime} \in \varphi^{-1}(B),
$$

donde $x \in \varphi^{-1}(A)+\varphi^{-1}(B)$, provando o item (iii).

Para o item $(v)$, é claro que

$$
x \in \psi^{-1} \psi(A) \Longleftrightarrow \psi(x) \in \psi(A) \Longleftrightarrow x \in A+\operatorname{ker} \psi,
$$

donde segue trivialmente também o item $(i v)$.

Finalmente, é claro que $\psi(A \cap B) \subseteq \psi(A) \cap \psi(B)$. Reciprocamente, digamos que $B \supseteq \operatorname{ker} \psi$. Se $x \in \psi(A) \cap \psi(B)$ então existem $a \in A$ e $b \in B$ tais que $x=\psi(a)=\psi(b)$. Daí $a-b \in \operatorname{ker} \psi$, ou seja, $a \in B+\operatorname{ker} \psi=B$. Daí segue que $x=\psi(a) \in \psi(A \cap B)$, provando (vi).

Lema 3.37. Para um $R$-módulo $M$ são equivalentes:

(i) Mé um D-módulo.

(ii) Para todo $R$-módulo $P$ e para todo $\varphi \in \operatorname{Hom}_{R}(P, M)$ temos

$$
\varphi^{-1}(A+B)=\varphi^{-1}(A)+\varphi^{-1}(B)
$$

para todos $A, B \in L_{R}(M)$.

(iii) Para todo R-módulo $Q$ e para todo $\psi \in \operatorname{Hom}_{R}(M, Q)$ temos

$$
\psi(A \cap B)=\psi(A) \cap \psi(B)
$$

para todos $A, B \in L_{R}(M)$.

Prova.

(i) $\Rightarrow(i i)$ Como $M$ é um D-módulo, pelo lema anterior podemos escrever

$$
\begin{aligned}
\varphi^{-1}(A)+\varphi^{-1}(B) & =\varphi^{-1} \varphi \varphi^{-1}(A)+\varphi^{-1} \varphi \varphi^{-1}(B) \\
& =\varphi^{-1}\left[\varphi \varphi^{-1}(A)+\varphi \varphi^{-1}(B)\right] \\
& =\varphi^{-1}[(A \cap \operatorname{im} \varphi)+(B \cap \operatorname{im} \varphi)] \\
& =\varphi^{-1}[(A+B) \cap \operatorname{im} \varphi] \\
& =\varphi^{-1} \varphi \varphi^{-1}(A+B) \\
& =\varphi^{-1}(A+B) .
\end{aligned}
$$


(ii) $\Rightarrow\left(\right.$ (i) Dado $S \in L_{R}(M)$ arbitrário, considere a inclusão $\iota: S \rightarrow M$. Então, para todos $A, B \in L_{R}(M)$ temos

$$
(A+B) \cap S=\iota^{-1}(A+B)=\iota^{-1}(A)+\iota^{-1}(B)=(A \cap S)+(B \cap S) .
$$

(i) $\Rightarrow($ iii $)$ Como $M$ é um D-módulo, pelo lema anterior podemos escrever

$$
\begin{aligned}
\psi(A) \cap \psi(B) & =\psi \psi^{-1} \psi(A) \cap \psi \psi^{-1} \psi(B) \\
& =\psi\left[\psi^{-1} \psi(A) \cap \psi^{-1} \psi(B)\right] \\
& =\psi[(A+\operatorname{ker} \psi) \cap(B+\operatorname{ker} \psi)] \\
& =\psi[(A \cap B)+\operatorname{ker} \psi] \\
& =\psi \psi^{-1} \psi(A \cap B) \\
& =\psi(A \cap B) .
\end{aligned}
$$

(iii) $\Rightarrow\left(\right.$ i) Dado $S \in L_{R}(M)$ arbitrário, tome a projeção $\pi: M \rightarrow M / S$. Então, para todos $A, B \in L_{R}(M)$ temos

$$
(A \cap B)+S=\pi(A \cap B)=\pi(A) \cap \pi(B)=(A+S) \cap(B+S),
$$

como queríamos demonstrar.

Proposição 3.38. Sejam $P$ e $Q$ dois R-módulos e $\varphi, \psi \in \operatorname{Hom}_{R}(P, Q)$.

(i) Se $Q$ é um D-módulo, então $P=\psi^{-1}(\operatorname{im} \varphi)+\varphi^{-1}(\operatorname{im} \psi)$.

(ii) Se P é um D-módulo, então $0=\psi(\operatorname{ker} \varphi) \cap \varphi(\operatorname{ker} \psi)$.

\section{Prova.}

(i) Como $P=(\varphi+\psi)^{-1}(\operatorname{im}(\varphi+\psi))$ e $\operatorname{im}(\varphi+\psi) \subseteq \operatorname{im} \varphi+\operatorname{im} \psi$, então $P=(\varphi+\psi)^{-1}(\operatorname{im} \varphi+\operatorname{im} \psi)$. Aplicando o lema (3.37) (ii), temos

$$
\begin{aligned}
P & =(\varphi+\psi)^{-1}(\operatorname{im} \varphi+\operatorname{im} \psi) \\
& =(\varphi+\psi)^{-1}(\operatorname{im} \varphi)+(\varphi+\psi)^{-1}(\operatorname{im} \psi) \\
& =\psi^{-1}(\operatorname{im} \varphi)+\varphi^{-1}(\operatorname{im} \psi) .
\end{aligned}
$$

(ii) Como $0=(\varphi+\psi)(\operatorname{ker}(\varphi+\psi))$ e $\operatorname{ker}(\varphi+\psi) \supseteq \operatorname{ker} \varphi \cap \operatorname{ker} \psi$, então $0=(\varphi+\psi)(\operatorname{ker} \varphi \cap \operatorname{ker} \psi)$. Aplicando o lema (3.37) (iii), temos

$$
\begin{aligned}
0 & =(\varphi+\psi)(\operatorname{ker} \varphi \cap \operatorname{ker} \psi) \\
& =(\varphi+\psi)(\operatorname{ker} \varphi) \cap(\varphi+\psi)(\operatorname{ker} \psi) \\
& =\psi(\operatorname{ker} \varphi) \cap \varphi(\operatorname{ker} \psi) .
\end{aligned}
$$


O leitor deste trabalho não terá deixado de perceber uma forte dualidade na maioria das definições e dos resultados deste capítulo; isso é conseqüência direta das condições equivalentes impostas para a distributividade de um reticulado (3.14). Essa dualidade é evidente não só nos enunciados, como também nas demonstrações; com efeito, a maioria das demonstrações de itens "duais" é levada a cabo invertendo o sentido de implicações e permutando os símbolos $\cap \mathrm{e}+$, im e ker, $\subseteq$ e $\supseteq$ etc.

Definição 3.39. Para quaisquer elementos $x, y$ de um $R$-módulo $M$, o ideal à esquerda $\{r \in R: r y \in R x\} \subseteq R$ será denotado por $(R x: y)$.

Lema 3.40. Se $R$ é um anel e $M$ um $R$-módulo, são equivalentes:

(i) $M$ é um D-módulo.

(ii) $(R a: b)+(R b: a)=R$ para todos $a, b \in M$.

(iii) $R(a+b)=[R a \cap R(a+b)]+[R b \cap R(a+b)]$ para todos $a, b \in M$.

\section{Prova.}

(i) $\Rightarrow$ (ii) Defina $P={ }_{R} R$ e $Q={ }_{R} M$, e considere os $R$-homomorfismos $\alpha, \beta \in \operatorname{Hom}_{R}(P, Q)$ dados por $\alpha(r)=$ ra e $\beta(r)=r b, \forall r \in R$. Note que

$$
\beta^{-1}(\operatorname{im} \alpha)=\beta^{-1}(R a)=\{r \in R: \beta(r) \in R a\}=(R a: b) ;
$$

similarmente temos também $\alpha^{-1}(\operatorname{im} \beta)=(R b: a)$. Pela proposição (3.38)(i) concluímos, portanto, que $(R a: b)+(R b: a)=R$ para todos $a, b \in M$.

(ii) $\Rightarrow$ (iii) Primeiro vejamos que $(R a:(a+b))(a+b)=R a \cap R(a+b)$ para todos $a, b \in M$. De fato, $r \in(R a:(a+b)) \Leftrightarrow r(a+b) \in R a$ por definição, donde $r(a+b) \in(R a:(a+b))(a+b) \subseteq R a \cap R(a+b)$ para todos $a, b \in M$. Por outro lado, se $m \in R a \cap R(a+b)$ para todos $a, b \in M$ então $m=r a=r^{\prime}(a+b)$ para algum $r^{\prime} \in R$, donde $r^{\prime} \in(R a:(a+b))$. Portanto $m=r^{\prime}(a+b) \in(R a:(a+b))(a+b)$, provando a igualdade desejada.

Em segundo lugar temos, como conseqüência direta da definição (3.39), as igualdades $(R a: b)=(R a:(a+b))$ e $(R b: a)=(R b:(a+b))$.

Portanto, se $(R a: b)+(R b: a)=R$ para todos $a, b \in M$, então

$$
\begin{aligned}
R & =(R a: b)+(R b: a) \quad \forall a, b \in M \\
\Longrightarrow \quad R(a+b) & =[(R a: b)+(R b: a)](a+b) \quad \forall a, b \in M \\
\Longrightarrow \quad R(a+b) & =[(R a:(a+b))+(R b:(a+b))](a+b) \quad \forall a, b \in M \\
\Longrightarrow \quad R(a+b) & =[R a \cap R(a+b)]+[R b \cap R(a+b)] \quad \forall a, b \in M .
\end{aligned}
$$


(iii) $\Rightarrow$ (i) Considere submódulos quaisquer $A, B, C \in L_{R}(M)$ e tome um elemento $c=a+b \in C \cap(A+B)$, onde $a \in A, b \in B$ e $c \in C$. Por hipótese

$$
R c=R(a+b)=(R a \cap R c)+(R b \cap R c) \subseteq(A \cap C)+(B \cap C),
$$

donde $C \cap(A+B) \subseteq(A \cap C)+(B \cap C)$. A inclusão contrária é óbvia.

O socle de um $R$-módulo $M$ é denotado por $\operatorname{soc}(M)$ e é, por definição, a soma de todos os $R$-submódulos simples de $M$. O resultado abaixo, devido a Victor Camillo [CA], nos dá uma caracterização simples de D-módulos em termos de socles de seus quocientes.

Teorema 3.41 (Camillo - 1975). Sejam $R$ um anel e $M$ um R-módulo. Então $M$ é um $D$-módulo se e somente se todo quociente de $M$ tem socle livre de quadrados, ou seja, $M / N$ nâo tem $R$-submódulos isomorfos a $T \oplus T$, onde $T$ é um $R$-módulo simples, para todo $R$-submódulo $N \subseteq M$.

Prova. Suponha que $M$ é um D-módulo e tome $N \in L_{R}(M)$. Se $M / N$ tivesse um $R$-submódulo da forma $T \oplus T$, onde $T$ é simples, $T \oplus T$ seria distributivo pela proposição (3.21). Mas isso contradiz a proposição (3.34) (e também a proposição 3.22 ), logo todo quociente de $M$ tem socle livre de quadrados.

Reciprocamente, suponha que todo quociente de $M$ tem socle livre de quadrados, e considere $a, b \in M$. Para provar que $M$ é um D-módulo veremos que $(R a: b)+(R b: a)=R$, e o resultado seguirá do lema (3.40).

Seja $K$ um ideal à esquerda maximal de $R$, e considere o $R$-módulo

$$
S=\frac{R a+R b}{K a+K b} .
$$

Se $a \in(K a+K b)$ então $(1-k) a \in K b \subseteq R b$ para algum $k \in K$. Portanto $(1-k) \in(R b: a)$, e como $(1-k) \notin K$ então $(R b: a) \subsetneq K$. Portanto $(R a: b)+(R b: a) \subsetneq K$. Como $K$ é arbitrário, isso significa que o ideal à esquerda $(R a: b)+(R b: a)$ não está contido em nenhum ideal à esquerda maximal de $R$, ou seja, $(R a: b)+(R b: a)=R$. De forma análoga, mostra-se que se $b \in(K a+K b)$ então $(R a: b)+(R b: a)=R$.

Suponha, agora, que $a \notin(K a+K b)$ e $b \notin(K a+K b)$. Como $K$ é um ideal à esquerda maximal, temos que

$$
A=\frac{R a+K b}{K a+K b} \quad \text { e } \quad B=\frac{K a+R b}{K a+K b}
$$

são $R$-submódulos simples de $S$, gerados, respectivamente, pelos elementos $a+(K a+K b)$ e $b+(K a+K b)$. Denote por $\bar{a}, \bar{b}$ as classes de $a, b$ em $S$ e por 
$\operatorname{Ann}_{R}(\bar{a})=\{r \in R: r \bar{a}=\overline{0}\}$ o anulador de $\bar{a}$ em $R$. Admita que $r_{1} \bar{a}=r_{2} \bar{a}$ para alguns $r_{1}, r_{2} \in R$ e suponha que $r_{1} \bar{b} \neq r_{2} \bar{b}$. Teríamos $\left(r_{1}-r_{2}\right) \bar{b} \neq \overline{0}$ e logo $\left(r_{1}-r_{2}\right) \notin K$, donde $\operatorname{Ann}_{R}(\bar{a}) \supseteq K+R\left(r_{1}-r_{2}\right)=R$, pois $K$ é maximal. Isso contradiria a hipótese de que $a \notin(K a+K b)$. Logo, mostramos que

$$
r_{1} \bar{a}=r_{2} \bar{a} \Longrightarrow r_{1} \bar{b}=r_{2} \bar{b} .
$$

Isso quer dizer que a aplicação $\varphi: A \rightarrow B$ dada por $\varphi(r \bar{a})=r \bar{b}$ para todo $r \in R$ está bem definida. É fácil ver que $\varphi$ é um isomorfismo, $\log 0 A \simeq B$.

Se $A \neq B$ então $\operatorname{soc}(M)$ teria um submódulo $A \oplus B \simeq A \oplus A$, contra a hipótese de $\operatorname{soc}(M)$ ser livre de quadrados. Então $A=B$, donde deduzimos que $a+(K a+K b)=r b+(K a+K b)$ para algum $r \in R$. Como feito acima, $(1-k) \in(R b: a)$ para algum $k \in K$, e temos $(R a: b)+(R b: a)=R$. Portanto, em qualquer caso temos $(R a: b)+(R b: a)=R$, como queríamos demonstrar.

À página 33 definimos o conceito de submódulo supérfluo. Definimos, agora, o conceito dual.

Definição 3.42 (ideais e submódulos essenciais). $P \in L_{R}(M)$ é dito um submódulo essencial do $R$-módulo $M$ se $P \cap Q \neq 0$ para todo submódulo não-nulo $Q \in L_{R}(M)$. Se todo $R$-submódulo näo-nulo de $M$ é essencial, dizemos que $M$ é um módulo uniforme. Um ideal à esquerda essencial de um anel $R$ é um $R$-submódulo essencial de ${ }_{R} R$.

Lema 3.43. Sejam $P$ e $Q$ dois $R$-módulos e $\varphi, \psi \in \operatorname{Hom}_{R}(P, Q)$.

(i) Suponha que todo submódulo próprio de $P$ é supérfluo e que $Q$ é um $D$-módulo. Então ou $\operatorname{im} \varphi \subseteq \operatorname{im} \psi$ ou $\operatorname{im} \psi \subseteq \operatorname{im} \varphi$.

(ii) Suponha que todo submódulo próprio de $Q$ é essencial e que $P$ é um $D$-módulo. Então ou $\operatorname{ker} \varphi \subseteq \operatorname{ker} \psi$ ou $\operatorname{ker} \psi \subseteq \operatorname{ker} \varphi$.

\section{Prova.}

(i) Pela proposição $3.38(i)$, temos $P=\psi^{-1}(\operatorname{im} \varphi)+\varphi^{-1}(\operatorname{im} \psi)$. Como todo $R$-submódulo de $P$ é supérfluo por hipótese, então $\psi^{-1}(\operatorname{im} \varphi)=P$ ou $\varphi^{-1}(\operatorname{im} \psi)=P$. Daí é claro que $\operatorname{im} \psi \subseteq \operatorname{im} \varphi$ ou $\operatorname{im} \varphi \subseteq \operatorname{im} \psi$.

(ii) Pela proposição $3.38($ ii $)$, temos que $0=\psi(\operatorname{ker} \varphi) \cap \varphi(\operatorname{ker} \psi)$. Como todo $R$-submódulo de $Q$ é essencial por hipótese, então $\psi(\operatorname{ker} \varphi)=0$ ou $\varphi(\operatorname{ker} \psi)=0$. Daí é claro que $\operatorname{ker} \varphi \subseteq \operatorname{ker} \psi$ ou $\operatorname{ker} \psi \subseteq \operatorname{ker} \varphi$.

Corolário 3.44. Se Ré um anel local $e_{R} M$ é um D-módulo, então $L_{R}(M)$ é um reticulado totalmente ordenado. 
Prova. Considere um elemento $m \in M$ arbitrário. O $R$-submódulo cíclico $R m \subseteq M$ é imagem da aplicação $\varphi_{m}: R \rightarrow M$ definida por $\varphi_{m}(r)=r m$ $\forall r \in R$. É claro que $\varphi_{m} \in \operatorname{Hom}_{R}(R, M)$; como $R$ é um anel local e ${ }_{R} M$ é um $D$-módulo, pelo lema (3.43) concluímos que os submódulos cíclicos de ${ }_{R} M$ formam uma cadeia totalmente ordenada.

Suponha que $L_{R}(M)$ não seja totalmente ordenado. Então existiriam $A, B \in L_{R}(M)$ tais que $A \not \subset B$ e $B \not \subset A$, donde existiriam $a \in A, b \in B$ tais que $a \notin B$ e $b \notin A$. No entanto, temos que $R a \subseteq R b$ ou $R b \subseteq R a$; digamos que seja $R a \subseteq R b$. Em particular, $a \in R b \subseteq B$, contradição. Logo, $L_{R}(M)$ é totalmente ordenado.

Corolário 3.45. $R$ é um anel local e distributivo à esquerda se, e somente se, $R$ é um anel uniserial à esquerda.

O teorema (3.46) abaixo, o resultado principal desta seção, estabelece que toda soma direta finita de anéis uniseriais pode ser caracterizada de maneira equivalente como um D-anel com mais alguma propriedade adicional (ser perfeito, semiperfeito ou artiniano).

Esse teorema foi provado por W. Stephenson em [ST I], generalizando assim o teorema (3.30), que trata do caso noetheriano.

Teorema 3.46 (Stephenson - 1974). Seja $R$ um anel.

(a) São equivalentes as seguintes afirmações:

(i) $R$ é um anel semiperfeito e distributivo à esquerda.

(ii) $R$ é soma direta finita de anéis uniseriais à esquerda.

(b) São equivalentes as seguintes afirmações:

(i) $R$ é um anel perfeito à esquerda e distributivo à esquerda.

(ii) $R$ é um anel perfeito à direita e distributivo à esquerda.

(iii) $R$ é um anel artiniano à esquerda e distributivo à esquerda.

(iv) $R$ é soma direta finita de anéis uniseriais à esquerda artinianos à esquerda.

Prova.

Parte (a)

(i) $\Rightarrow$ (ii) $R$ é um anel semiperfeito, $\log R$ admite uma decomposição $R=S \oplus M$ tal como descrita no teorema (2.22). Como $R$ é um D-anel, todo idempotente de $R$ é central pelo corolário (3.35). Então $M=0 \mathrm{e}$ $R=\bigoplus_{i=1}^{t} R e_{i}$, sendo $\left\{e_{1}, e_{2}, \ldots, e_{t}\right\}$ uma família completa de idempotentes 
primitivos ortogonais centrais em $R$. Pelo teorema (2.25) esses idempotentes são locais, de modo que $R$ é uma soma direta finita de anéis locais. Cada componente $R e_{i}$ dessa soma é um D-anel à esquerda. De fato, se $I$ é um ideal à esquerda de $R e_{i}$ então $I=I e_{i}=e_{i} I$ e temos

$$
R e_{i} \cdot I \subseteq I \Longrightarrow R \cdot e_{i} I \subseteq I \Longrightarrow R I \subseteq I
$$

donde $I$ é um ideal à esquerda de $R$. Assim, o reticulado de ideais à esquerda de $R e_{i}$ é um subreticulado do reticulado distributivo $L_{R}\left({ }_{R} R\right)$. Daí temos que $R$ é uma soma direta finita de anéis locais distributivos à esquerda, que são anéis uniseriais à esquerda pelo corolário (3.45).

(ii) $\Rightarrow$ (i) Suponha que $R=S_{1} \oplus \cdots \oplus S_{t}$ é uma soma direta finita de anéis uniseriais à esquerda. Pelo corolário (3.45), cada componente dessa soma é um anel local distributivo à esquerda. É claro que $1=e_{1}+\cdots+e_{t}$ é uma decomposição da unidade de $R$ em uma soma finita de idempotentes ortogonais não-nulos $e_{1}, \cdots, e_{t}$. Como os $S_{i}$ são anéis locais, pela proposição (2.2) só possuem idempotentes triviais; logo, cada $e_{i}$ é a unidade do anel $S_{i}$. Então $e_{i} S_{i} e_{i}=S_{i}$ é um anel local, donde os $e_{i}$ são idempotentes locais e portanto 1 é soma finita de idempotentes locais ortogonais. Pelo teorema (2.28), $R$ é semiperfeito. Pela proposição (3.20), $R$ é também um D-anel à esquerda.

Parte (b)

$(i v) \Rightarrow$ (iii) Se $R$ é soma direta de um número finito de anéis uniseriais à esquerda artinianos à esquerda, então $R$ é um anel artiniano à esquerda. Como anéis uniseriais à esquerda são distributivos à esquerda e somas diretas de D-anéis são D-anéis pela proposição (3.20), segue o resultado.

$($ iii $) \Rightarrow(i i),(i i i) \Rightarrow(i)$ Isso é claro (cf. exemplo 2.60$)$

$(i i) \Rightarrow(i v)$ Como todo anel perfeito à direita é semiperfeito, podemos dizer, pelo item (a) acima, que $R$ é soma direta finita $R_{1} \oplus \cdots \oplus R_{t}$ de anéis uniseriais à esquerda. Se $R$ é perfeito à direita, então cada $R_{i}$ satisfaz a $\mathrm{CCD}$ em ideais à esquerda principais. Suponha que $A_{1} \supset A_{2} \supset A_{3} \supset \ldots$ é uma cadeia estritamente descendente de ideais à esquerda de $R_{i}$. Escolha $a_{i} \in A_{i}$ tal que $a_{i} \notin A_{i+1}$; como $R$ é uniserial à esquerda, $A_{i} \supseteq R a_{i} \supset A_{i+1}$. Portanto, obtemos uma cadeia estritamente descendente de ideais à esquerda principais em $R_{i}$, uma contradição. Então cada $R_{i}$ é artiniano à esquerda.

$(i) \Rightarrow(i v)$ Ainda pelo item (a) acima, podemos dizer que $R$ é soma direta finita $R_{1} \oplus \cdots \oplus R_{t}$ de anéis uniseriais à esquerda. Se $R$ é perfeito à esquerda, 
então cada $R_{i}$ satisfaz a $\mathrm{CCD}$ em ideais à direita principais. Por um resultado bastante técnico de David Jonah [JO], cada $R_{i}$ satisfaz a $C C A$ em ideais à esquerda principais. Como cada $R_{i}$ é uniserial à esquerda, por um argumento análogo ao que foi feito na implicação acima segue que $R_{i}$ é noetheriano à esquerda. Então $J_{i}=J\left(R_{i}\right)$ é nilpotente pelo teorema de Levitzki (1.33), donde cada $R_{i}$ é semiprimário. Então, o teorema de Hopkins-Levitzki (1.34) nos diz que cada $R_{i}$ é artiniano à esquerda, como queríamos demonstrar. 


\section{Capítulo 4}

\section{Órbitas sob a Ação Regular}

Neste último capítulo, apresentaremos uma aplicação recente dos teoremas de Stephenson e Camilllo: a caracterização completa de anéis que têm apenas um número finito de órbitas sob a ação regular à esquerda. Esta classificação foi obtida por Hirano [HI] em 2002.

$\mathrm{Na}$ seção 4.1 são apresentados conceitos e proposições preliminares, e o teorema de Hirano é demonstrado. Na seção 4.2 são apresentados alguns exemplos.

\subsection{Teorema de Hirano}

Começamos nossa exposição relembrando o conceito de ação de um grupo.

Definição 4.1 (ações de grupos). Sejam $G$ um grupo e $A$ um conjunto. Uma ação de $G$ sobre $A$ é uma aplicação $\mu: G \times A \rightarrow A$, escrita $\mu(g, a)=g \cdot a$ para todos $g \in G$ e $a \in A$, tal que

(i) $g_{1} \cdot\left(g_{2} a\right)=\left(g_{1} g_{2}\right) \cdot a \quad \forall g_{1}, g_{2} \in G$;

(ii) $1 \cdot a=a \quad \forall a \in A$.

Se $\mu: G \times A \rightarrow A$ é uma ação de $G$ sobre $A$, diremos que $G$ age sobre $A$.

É fácil ver que se $G$ é um grupo agindo sobre um conjunto $A$ então a relação $\sim$ em $A$ definida por $a \sim b \Leftrightarrow a=g b$ para algum $g \in G$ é uma relação de equivalência em $A$. Estaremos interessados nas classes de equivalência definidas por essa relação.

Definição 4.2 (órbitas). Seja $G$ um grupo agindo sobre um conjunto $A$. As classes de equivalência determinadas pela relação $\sim$ mencionada acima são chamadas órbitas da ação. A órbita de um elemento $x \in A$ é a classe de equivalência que o contém, e é denotada por $\mathcal{O}(x)$. 
Definição 4.3 (a ação regular à esquerda). Seja $R$ um anel com unidade e $U=U(R)$ o conjunto de todos os elementos invertiveis de $R$. A aplicação que a cada $(u, r) \in U \times R$ associa o produto $u \cdot r$ é uma ação, denominada ação regular à esquerda. As órbitas dessa ação são também chamadas de U-órbitas.

Proposição 4.4. Seja $R$ um anel com unidade que tem um número finito de órbitas sob a ação regular à esquerda. Então $R$ tem um número finito de

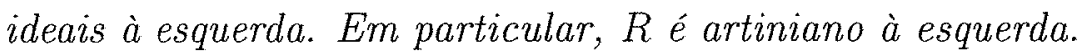

Prova. Todo ideal à esquerda de $R$ é invariante pela ação regular à esquerda, pois se $L \subseteq R$ é um ideal à esquerda então $U L \subseteq R L \subseteq L$. Veremos que todo ideal à esquerda de $R$ é uma união disjunta de $U$-órbitas. De fato, é fácil ver que

$$
a \in \mathcal{O}(x) \Longleftrightarrow a=u x \text { para algum } u \in U \Longleftrightarrow a \in U x
$$

$\operatorname{logo} \mathcal{O}(x)=U x$ para todo $x \in R$. Então

$$
\left.\begin{array}{r}
\bigcup_{x \in L} \mathcal{O}(x)=\bigcup_{x \in L} U x=U L \subseteq L \\
L=\bigcup_{x \in L}\{x\}=\bigcup_{x \in L}\{1 \cdot x\} \subseteq \bigcup_{x \in L} U x=\bigcup_{x \in L} \mathcal{O}(x)
\end{array}\right\} \Longrightarrow L=\bigcup_{x \in L} \mathcal{O}(x)
$$

Provamos que todo ideal à esquerda de $R$ é uma união disjunta de $U$-órbitas (pois órbitas são sempre disjuntas), $\operatorname{logo} R$ possui um número finito de ideais à esquerda distintos, donde é claro que $R$ é artiniano à esquerda.

Proposição 4.5. Sejam $R$ um anel que tem um número finito de ideais à esquerda, e $J=J(R)$. Então $R / J$ é isomorfo a uma soma direta de um número finito de anéis de divisão e um anel finito.

Prova. Pela proposição (4.4) $R$ é artiniano à esquerda, portanto $R / J$ é um anel artiniano semisimples pela proposição (1.24). Pelo teorema de Wedderburn-Artin, $R / J$ é isomorfo a uma soma direta de anéis de matrizes sobre anéis de divisão, cada qual um anel artiniano simples. Seja $S \simeq M_{n}(D)$ uma das componentes simples de $R / J$ para algum inteiro positivo $n$ e algum anel de divisão $D$. É fácil ver que se $D$ tem infinitos elementos e $n \geqslant 2$ então $M_{n}(D)$ tem infinitos ideais à esquerda (por exemplo, se $a, b \in D$ são distintos então os ideais à esquerda de $M_{n}(D)$ gerados pelos elementos $E_{11}+a E_{12} \mathrm{e}$ $E_{11}+b E_{12}$ também são distintos). Portanto, por hipótese, ou $D$ é finito ou $n=1$, ou seja, cada componente simples de $R / J$ é um anel de divisão ou é um anel finito. 
Proposição 4.6. Seja $R$ um anel que tem apenas um número finito de ideais à esquerda. Então nenhum quociente de ${ }_{R} R$ tem submódulos isomorfos a $T \oplus T$, sendo $T$ um $R$-módulo simples com infinitos elementos.

Prova. Suponha, por absurdo, que existam em $R$ ideais à esquerda $L, A$ e $B$ tais que $R / L$ tenha um submódulo

$$
\frac{A}{L} \oplus \frac{B}{L},
$$

sendo $A / L \simeq B / L$ dois $R$-módulos simples isomorfos com infinitos elementos. Pela proposição (4.5) podemos escrever

$$
\frac{R}{J} \simeq D_{1} \oplus \cdots \oplus D_{m} \oplus F,
$$

sendo cada $D_{i}$ um anel de divisão infinito e $F$ um anel semisimples finito.

Como $A / L$ é um $R$-módulo simples, $J \subseteq A n n_{R}(A / L)$ por definição. Logo, pela proposição (1.7), $A / L$ admite uma estrutura de $R / J$-módulo, e como $A / L$ é simples como $R$-módulo, então é simples também como $R / J$-módulo. Pela proposição (1.27), $A / L$ é isomorfo a algum ideal à esquerda minimal de $R / J$. Como $F$ é finito e $A / L$ tem infinitos elementos podemos supor, sem perda de generalidade, que $A / L \simeq D_{1}$ como $R / J$-módulos. Claramente temos, também, que $A / L \simeq D_{1}$ como $R$-módulos. Logo $A / L \simeq D_{1} \simeq B / L$ e então $A / L \oplus B / L$ tem, naturalmente, uma estrutura de espaço vetorial à esquerda sobre $D_{1}$.

Considere, agora, a projeção natural $\pi: R \rightarrow D_{1}$. Não é difícil ver que

$$
\text { ker } \pi=\operatorname{Ann}_{R}\left(\frac{A}{L} \oplus \frac{B}{L}\right),
$$

$\log 0$

$$
\frac{R}{\operatorname{Ann}_{R}\left(\frac{A}{L} \oplus \frac{B}{L}\right)} \simeq D_{1}
$$

pelo primeiro teorema do isomorfismo. Pela proposição (1.7), o reticulado $L_{1}$ de $R$-submódulos de $A / L \oplus B / L$ é isomorfo ao reticulado $L_{2}$ de subespaços do espaço vetorial $A / L \oplus B / L$ sobre $D_{1}$. No entanto, como este espaço é de dimensão 2 sobre um anel de divisão infinito, $L_{2}$ é infinito; como $R$ tem um número finito de ideais à esquerda por hipótese, $L_{1}$ é um reticulado finito. Dessa contradição, deduzimos que nenhum quociente de ${ }_{R} R$ tem submódulos isomorfos a $T \oplus T$, sendo $T$ um $R$-módulo simples infinito. 
O teorema de Hirano estabelece a equivalência entre número finito de órbitas sob a ação regular à esquerda e número finito de ideais à esquerda, ao mesmo tempo descrevendo explicitamente a estrutura de anéis com essa propriedade.

Teorema 4.7 (Hirano - 2002). Se $R$ é um anel, são equivalentes:

(i) R tem um número finito de órbitas sob a ação regular à esquerda.

(ii) R tem um número finito de ideais à esquerda.

(iii) $R$ é soma direta de um número finito de anéis uniseriais à esquerda artinianos à esquerda mais um anel finito.

\section{Prova.}

$(i) \Rightarrow(i i)$ Proposição (4.4).

(ii) $\Rightarrow$ (iii) Suponha que $R$ tem um número finito de ideais à esquerda. Então $R$ é artiniano à esquerda e, pela proposição (4.5), temos

$$
R / J=D_{1} \oplus \cdots \oplus D_{k} \oplus F_{1} \oplus \cdots \oplus F_{m}
$$

sendo cada $D_{i}$ um anel de divisão infinito e cada $F_{j}$ um anel simples finito. Suponha que $k \geqslant 1$ e $m \geqslant 1$. Pelo teorema (1.40), $\bar{R}=R / J$ tem uma família completa de idempotentes ortogonais centrais

$$
\left\{\bar{e}_{1}, \ldots, \bar{e}_{k}, \bar{f}_{1}, \ldots, \bar{f}_{m}\right\},
$$

sendo cada $\bar{e}_{i} \in D_{i}$ e cada $\bar{f}_{j} \in F_{j}$ os respectivos elementos identidade das componentes simples $D_{i}$ e $F_{j}, 1 \leqslant i \leqslant k, 1 \leqslant j \leqslant m$. Pelas proposições (2.17) e (2.18), esses idempotentes podem ser levantados a uma família completa de idempotentes ortogonais $\left\{e_{1}, \ldots, e_{k}, f_{1}, \ldots, f_{m}\right\}$ em $R$.

Suponha que $e_{p} R f_{q} \neq 0$ para alguns $p, q$ e tome $a \in R$ tal que $e_{p} a f_{q} \neq 0$. Podemos afirmar:

- $D_{p} \simeq \frac{R e_{p}}{J e_{p}}$ como $R$-módulos.

De fato, defina $\varphi: D_{p} \rightarrow R e_{p} / J e_{p}$ por $\varphi\left(r e_{p}+J\right)=r e_{p}+J e_{p} \forall r \in R$. É rotina verificar que $\varphi$ está bem definida e que $\varphi$ é um epimorfismo. Por outro lado, $r e_{p}+J e_{p}=0 \Rightarrow r e_{p} \in J e_{p} \Rightarrow r e_{p} \in J \Rightarrow r e_{p}+J=0$, donde $\varphi$ é também injetora. 
- $\frac{R e_{p}}{J e_{p}} \simeq \frac{R e_{p} a f_{q}}{J e_{p} a f_{q}}$ como $R$-módulos.

De fato, defina $\psi: r e_{p}+J e_{p} \longmapsto r e_{p} a f_{q}+J e_{p} a f_{q} \forall r \in R$. É rotina verificar que $\psi$ está bem definida e que $\psi$ é um epimorfismo, restando mostrar que $\psi$ é injetora. Como $D_{p} \simeq R e_{p} / J e_{p}$ é um $R$-módulo simples, ker $\psi$ só pode ser 0 ou $R e_{p} / J e_{p}$. Para mostrar que ker $\psi=0$ vejamos que $R e_{p} a f_{q} / J e_{p} a f_{q} \neq 0$. De fato, se fosse $e_{p} a f_{q} \in J e_{p} a f_{q}$ então teríamos $e_{p} a f_{q}=b e_{p} a f_{q}$ para algum $b \in J$, donde $(1-b) e_{p} a f_{q}=0$. Mas $1-b \in U(R)$ e $e_{p} a f_{q} \neq 0$ por hipótese, uma contradição; $\log 0 e_{p} a f_{q}+J e_{p} a f_{q} \neq 0$ como queríamos. Sendo assim, $T=R e_{p} a f_{q} / J e_{p} a f_{q}$ é um $R$-submódulo simples de $R f_{q} / J e_{p} a f_{q}$, donde

$$
\frac{R}{J e_{p} \oplus J e_{p} a f_{q}} \simeq \frac{R e_{p}}{J e_{p}} \oplus \frac{R f_{q}}{J e_{p} a f_{q}} \oplus\left(\bigoplus_{i \neq p} R e_{i}\right) \oplus\left(\bigoplus_{j \neq q} R f_{j}\right)
$$

é um quociente de ${ }_{R} R$ contendo um $R$-submódulo isomorfo a $T \oplus T$, sendo $T$ simples com infinitos elementos, em contradição com a proposição (4.6). Logo, $e_{i} R f_{j}=0$ para todos $i, j$.

Suponha, agora, que $f_{q} R e_{p} \neq 0$ para alguns índices $p, q$. Como grupos abelianos, podemos escrever

$$
\frac{\left(e_{p}+f_{q}\right) R\left(e_{p}+f_{q}\right)}{e_{p} J e_{p}+f_{q} J f_{q} R e_{p}+f_{q} R e_{p} J e_{p}+f_{q} J f_{q}} \simeq \frac{e_{p} R e_{p}}{e_{p} J e_{p}} \oplus M \oplus \frac{f_{q} R f_{q}}{f_{q} J f_{q}},
$$

onde

$$
M=\frac{f_{q} R e_{p}}{f_{q} J f_{q} R e_{p}+f_{q} R e_{p} J e_{p}} .
$$

Definindo $H=e_{p} J e_{p}, I=f_{q} J f_{q}$ e $N=f_{q} R e_{p}$ temos $M=N /(I N+N H)$. Suponha que $M=0$, isto é, que $N=I N+N H$. Como $I$ é nilpotente e $N \neq 0$ por hipótese, existe um inteiro positivo $n$ tal que $I^{n} N=0$ e $I^{n-1} N \neq 0$. Então

$$
I^{n-1} N=I^{n} N+I^{n-1} N H=I^{n-1} N H .
$$

Como $H$ é nilpotente e $I^{n-1} N \neq 0$, existe um inteiro positivo $m$ tal que $I^{n-1} N H^{m}=0$ e $I^{n-1} N H^{m-1} \neq 0$. No entanto, como $I^{n-1} N=I^{n-1} N H$ obtemos

$$
I^{n-1} N H^{m-1}=I^{n-1} N H H^{m-1}=I^{n-1} N H^{m}=0,
$$

uma contradição. Logo, $M \neq 0$. 
Observe que $M$ é um módulo à direita sobre o anel $e_{p} R e_{p}$, e que $e_{p} J e_{p}$ está contido em Ann $M_{e_{p} R e_{p}}$. Pela proposição (1.7), $M$ é um espaço vetorial à direita sobre o anel de divisão $e_{p} R e_{p} / e_{p} J e_{p} \simeq D_{p}$. Assim, $M$ é um espaço vetorial não-nulo sobre um anel de divisão com infinitos elementos, donde $M$ tem infinitos elementos. Observe também que $M$ é um módulo à esquerda sobre o anel $f_{q} R f_{q}$, e que $f_{q} J f_{q}$ está contido em $A n_{f_{q} R f_{q}} M$. Pela proposição (1.7), $M$ é um módulo à esquerda sobre o anel finito $f_{q} R f_{q} / f_{q} J f_{q} \simeq F_{q}$. Como $F_{q}$ é isomorfo a um anel de matrizes $M_{n}(D) \operatorname{com} n \geqslant 1$ e $D$ finito, $M$ é completamente redutivel e, assim, isomorfo a uma soma direta de ideais à esquerda (minimais) finitos de $M_{n}(D)$. Se essa soma direta fosse finita, $M$ seria finito, o que não é o caso; portanto, existe uma cadeia descendente infinita de submódulos distintos de $M, \log o M$ não é artiniano à esquerda. Como o anel $\left(e_{p}+f_{q}\right) R\left(e_{p}+f_{q}\right)$ é artiniano, isso contradiz a equação (4.1). Então, podemos afirmar que $f_{j} R e_{i}=0$ para todos $i, j$.

Escreva $e=\sum_{i=1}^{k} e_{i}$ e $f=\sum_{j=1}^{m} f_{j}$. Até agora, temos que $R=e R e \oplus f R f$ é uma soma direta de anéis. Então $J=J(R)=e J e \oplus f J f$. Como o anel $F=f R f$ é artiniano à esquerda, o módulo ${ }_{F} F$ tem uma série de composição

$$
0 \subset A_{1} \subset \cdots \subset A_{n}=F,
$$

e cada fator $A_{i+1} / A_{i}$ dessa série é um $F$-módulo simples. Assim, cada fator é um módulo simples sobre $F / J(F)$. No entanto, como

$$
F / J(F)=f R f / f J f \simeq F_{1} \oplus \cdots \oplus F_{m}
$$

é um anel finito, cada fator da série é um conjunto finito. Então é fácil ver que $F$ é finito.

Considere agora o anel $S=e R e$. Pela proposição (4.6), nenhum quociente de ${ }_{S} S$ tem submódulos isomorfos a $T \oplus T$, sendo $T$ um $S$-módulo simples com infinitos elementos. Mas todo $S$-módulo simples tem necessariamente infinitos elementos. De fato, se $T$ é um $S$-módulo simples então $T$ é também um $S / J(S)$-módulo simples; como $J(S)=e J$ e então $S / J(S)$ é isomorfo a $D_{1} \oplus \cdots \oplus D_{k}, \operatorname{logo} T$ é isomorfo a um dos $D_{i}$ pela proposição (1.27). Podemos, então, dizer que quocientes de ${ }_{S} S$ não têm submódulos isomorfos a $T \oplus T$, sendo $T$ um $S$-módulo simples. Pelo teorema de Camillo (3.41), ${ }_{S} S$ é um módulo distributivo. Como $S$ é artiniano à esquerda, pelo teorema de Stephenson (3.46) sabemos que $S$ é soma direta finita de anéis uniseriais à esquerda artinianos à esquerda.

Então $R=S \oplus F$ é soma direta de um número finito de anéis uniseriais à esquerda artinianos à esquerda mais um anel finito. 
$($ iii $) \Rightarrow($ i) Podemos admitir, sem perda de generalidade, que $R$ é um anel uniserial à esquerda artiniano à esquerda. Seja $R=I_{0} \supset \cdots \supset I_{n}=0$ a cadeia de todos os ideais à esquerda de $R$, e tome um elemento arbitrário $a_{i} \in I_{i} \backslash I_{i+1}$ para cada $i=0,1, \ldots, n-1$. São de fácil verificação as afirmações abaixo:

Fato 1: $x \in I_{0} \backslash I_{1} \Leftrightarrow R x=R$ e $x$ é invertível à esquerda.

Fato 2: $I_{1}=J(R)$.

Considere elementos $a_{i} \in I_{i} \backslash I_{i+1}$ para todo $i, 0 \leqslant i \leqslant n-1$. Vamos provar que $I_{i} \backslash I_{i+1}=U a_{i}$. É claro que $U a_{i} \subseteq I_{i} \backslash I_{i+1}$, pois se fosse $u a_{i} \in I_{i+1}$ para algum $u \in U$ teríamos $u^{-1} u a_{i}=a_{i} \in I_{i+1}$. Resta mostrar a inclusão contrária.

Para um certo índice $i$ suponha, por absurdo, que existe $b_{i} \in I_{i} \backslash I_{i+1}$ tal que $b_{i} \notin U a_{i}$. É claro que $U b_{i} \subseteq I_{i} \backslash I_{i+1}$ e $U a_{i}, U b_{i}$ são disjuntos, pois se $U a_{i} \cap U b_{i} \neq \varnothing$ então $u a_{i}=v b_{i}$ para certos $u, v \in U$ e daí $b_{i}=v^{-1} u a_{i} \in U a_{i}$. Observe que $I_{i}=R a_{i}=R b_{i}$. Então existem $r, s \in R \backslash U$ tais que

$$
\left\{\begin{array} { c } 
{ b _ { i } = r a _ { i } } \\
{ a _ { i } = s b _ { i } }
\end{array} \Longrightarrow \left\{\begin{array} { c } 
{ a _ { i } = ( s r ) a _ { i } } \\
{ b _ { i } = ( r s ) b _ { i } }
\end{array} \Longrightarrow \left\{\begin{array}{c}
(1-s r) a_{i}=0 \\
(1-r s) b_{i}=0
\end{array},\right.\right.\right.
$$

logo $(1-r s)$ e $(1-s r)$ não são invertíveis. Se mostrarmos que $r$ é invertível chegaremos a uma contradição, provando que $U a_{i}=I_{i} \backslash I_{i+1}$.

- $r, s, r s$ e $s r$ são invertíveis à esquerda.

Com efeito, se $r s \in I_{1}=J(R)$ então $(1-r s) \in U$ pelo teorema (1.24). Logo $r s \in I_{0} \backslash I_{1}$, donde $r s$ é invertível à esquerda pelo fato 1 . De forma análoga vemos que $s r$ é invertível à esquerda. Daí segue facilmente que $r$ e $s$ são invertíveis à esquerda.

A esta altura, a proposição (2.2) já nos diz que $r$ e $s$ são invertíveis, pois $R$ é um anel local. No entanto, vejamos como também é possível mostrar isso diretamente.

- rs e sr são invertíveis.

Como rs é invertível à esquerda, existe $t \in R$ tal que $t r s=1$, donde $t$ é invertível à direita. Como $r s b_{i}=b_{i}$ pela equação (4.2), vem

$\operatorname{trs}=1 \Rightarrow \operatorname{trs} b_{i}=b_{i} \Rightarrow t b_{i}=b_{i} \Rightarrow(1-t) b_{i}=0 \Rightarrow(1-t) \notin U \Rightarrow t \notin I_{1}=J(R)$.

Logo $t \in I_{0} \backslash I_{1}$, e portanto $t$ é invertível à esquerda pelo fato 1 . Vimos que $t$ é invertível à esquerda e à direita, logo $t \in U$ é invertível e temos que $r s=t^{-1} \in U$ é invertível. Um raciocínio inteiramente análogo nos mostra que também $s r$ é invertivel. 
- $r$ e $s$ são invertíveis.

Vimos que $r$ é invertível à esquerda. Como $t^{-1}=r s$ então $(r s) t=t(r s)=1$, donde $r$ é invertível à direita. Logo $r$ é invertível, e de forma análoga vemos que $s$ é invertível. $\operatorname{Logo} U a_{i}=I_{i} \backslash I_{i+1}, 0 \leqslant i \leqslant n-1$, donde segue facilmente que $R \backslash\{0\}=U a_{0} \cup \cdots \cup U a_{n}$. Pela prova da proposição (4.4) temos que $U a_{0}, \ldots, U a_{n}$ são todas as órbitas não-nulas de $R$ sob a ação regular à esquerda. Assim, chegamos à contradição desejada.

Corolário 4.8. Seja $R$ um anel que não tenha quocientes não-nulos finitos. Então, são equivalentes:

(i) $R$ tem um número finito de órbitas sob a ação regular à esquerda.

(ii) $R$ é um D-anel à esquerda, artiniano à esquerda.

Prova. Conseqüência direta do teorema (4.7).

Corolário 4.9. Seja $R$ um anel local infinito, e suponha que $R$ tem $n+2$ órbitas sob a ação regular à esquerda, e seja $J=J(R)$. Então $J^{n} \neq 0$, mas $J^{n+1}=0$.

Prova. Pelo teorema (4.7), $R$ é um anel uniserial à esquerda. Como $R$ tem $n+2$ órbitas sob a ação regular à esquerda, podemos ver facilmente que a cadeia de todos os ideais à esquerda de $R$ é $R \supseteq J \supseteq J^{2} \supseteq \cdots \supseteq J^{n+1}=0$. Logo $J^{n} \neq 0$.

\subsection{Exemplos}

Finalmente, calcularemos o número de órbitas sob a ação regular à esquerda para alguns anéis finitos. O primeiro exemplo mostra que a afirmação do corolário (4.9) não é verdadeira para anéis locais finitos.

\section{Exemplo 4.10.}

Sejam $F=\mathbb{F}_{q}$ o corpo de $q$ elementos e $V$ um espaço vetorial de dimensão finita $m$ sobre $F$. Considere o anel

$$
R=\left\{\|a, v\|=\left[\begin{array}{ll}
a & v \\
0 & a
\end{array}\right]: a \in F, v \in V\right\} .
$$

É fácil ver que $\|a, v\| \in U(R)$ se e somente se $a \neq 0$, e portanto $\|0, V\|$ é o único ideal à esquerda maximal de $R$. Logo $R$ é um anel local finito com $J=J(R)=\|0, V\|$. Duas órbitas de $R$ sob a ação regular à esquerda são 
$\{0\}$ e $U(R)$; como $\|a, v\| \cdot\|0, w\|=\|0, a w\|$ para todos $\|a, v\| \in U(R), w \in V$, vemos que as demais órbitas são da forma $\|0, S\|$, onde $S$ é um subespaço unidimensional de $V$. O espaço $V$ tem $q^{m}-1$ elementos não-nulos, e cada subespaço de dimensão 1 tem $q-1$ elementos não-nulos. Não é difícil ver que existem, portanto,

$$
n=\frac{q^{m}-1}{q-1}=1+q+q^{2}+\cdots+q^{m-1}
$$

subespaços unidimensionais de $V$. Então $R$ tem $n+2$ órbitas sob a ação regular à esquerda, mas $J^{n}=0$ se $m>1$. De fato, $J^{2}=0$.

Exemplo 4.11. Órbitas sob a ação regular para o anel $\mathbb{M}_{n}\left(\mathbb{F}_{q}\right)$.

Sejam $F=\mathbb{F}_{q}$ o corpo de $q$ elementos e $n$ um inteiro positivo. Considere o anel de matrizes $R=\mathbb{M}_{n}(F)$ sobre $F$. Nesse caso, $U(R)$ é o grupo linear geral $G=G L_{n}(F)$. Defina $E_{r}=E_{11}+\cdots+E_{r r}$. Se $A \in R \backslash\{0\}$, sabemos que existem $X, Y \in G$ tais que $X A Y=E_{r}$, ou seja, toda matriz de $R$ com posto $r$ é um elemento do conjunto $G E_{r} G$. Portanto podemos dizer que $R$ é a união disjunta dos conjuntos

$$
\{0\}, G E_{1} G, \ldots, G E_{n} G=G .
$$

É claro que, em cada órbita, todas as matrizes têm o mesmo posto. Então temos que contar o número de $G$-órbitas em cada um dos $G E_{r} G$. O conjunto de $G$-órbitas em $G E_{r} G$ é

$$
S_{r}=\left\{G E_{r} g_{1}, \ldots, G E_{r} g_{s}\right\} .
$$

Podemos supor, sem perda de generalidade, que $g_{1}=1$. Se considerarmos o subgrupo $H_{r}=\left\{g \in G: G E_{r} g=G E_{r}\right\} \subseteq G$, temos que $s=|G| /\left|H_{r}\right|$. Portanto, o número de órbitas sob a ação regular à esquerda é

$$
1+\sum_{r=1}^{n} \frac{|G|}{\left|H_{r}\right|}
$$

Para calcular esse número, veja que $H_{r}$ pode ser determinado explicitamente:

$$
H_{r}=\left\{\left[\begin{array}{cc}
A & 0 \\
C & B
\end{array}\right]: A \in G L_{r}(F), B \in G L_{n-r}(F), C \in \mathbb{M}_{(n-r) \times r}(F)\right\} .
$$

Portanto $\left|H_{r}\right|=\left[\left(q^{r}-1\right) \cdots\left(q^{r}-q^{r-1}\right)\right]\left[q^{r(n-r)}\right]\left[\left(q^{n-r}-1\right) \cdots\left(q^{n-r}-q^{n-r-1}\right)\right]$, e então o número de órbitas de $R$ sob a ação regular à esquerda é

$1+\sum_{r=1}^{n}|G| /\left|H_{r}\right|=1+\sum_{r=1}^{n}\left[\left(q^{n}-1\right) \cdots\left(q^{n}-q^{r-1}\right)\right] /\left[\left(q^{r}-1\right) \cdots\left(q^{r}-q^{r-1}\right)\right]$. 
Nosso último objetivo é construir um anel que tem um número finito de órbitas sob a ação regular à esquerda, mas infinitas órbitas sob a ação regular à direita. Para tanto, precisamos de algumas definições e alguns resultados preliminares.

Definição 4.12 (anéis de polinômios skew). Sejam $D$ um anel de divisão, $\sigma \in \operatorname{End}(D)$ um endomorfismo e $x$ uma variável. $O$ anel $R=D[x ; \sigma]$ é definido como o grupo abeliano de polinômios formais $\sum_{i} a_{i} x^{i}$ munido de uma multiplicação definida por xa $=\sigma(a) x$ para todo $a \in D$, estendida distributivamente a todo $R$. O grau de um polinômio $f$ será denotado por $\operatorname{deg} f$.

Proposição 4.13. Seja $D$ um anel de divisão. Então $T=D[x ; \sigma]$ é um domínio ideal à esquerda principal (DIEP). Além disso, se $T f=T g$ então $f=d g$ para algum $d \in D$. Em particular, $f$ e g têm o mesmo grau e o mesmo número de termos não-nulos.

Prova. Um ideal à esquerda $L \subseteq T$ é gerado por qualquer polinômio $g \in L$ de grau minimal. De fato, se $f \in L$ então a divisão de $f$ por $g$ nos permite escrever $f=q_{1} g+r$; pela minimalidade de $\operatorname{deg} g$ temos $r=0$, donde $f \in T g$. Se $T f=T g$ então $g=q_{2} f$ e portanto $\operatorname{deg} f \leqslant \operatorname{deg} g$. Então é claro que $\operatorname{deg} f=\operatorname{deg} g$ e $q_{1}=d \in D$.

Proposição 4.14. Para um anel $T=D[x ; \sigma]$, são equivalentes:

(i) $\sigma$ é epimorfismo.

(ii) T é um domínio ideal à direita principal (DIDP).

(iii) Se $f, g \in T \backslash\{0\}$ então $f T \cap g T \neq 0$.

Observação: (iii) é conhecida como "right Ore condition" . Prova.

$(i) \Rightarrow(i i)$ Se $\sigma$ é epimorfismo então todo elemento $\sum a_{i} x^{i} \in T$ pode ser escrito na forma $\sum x^{i} \sigma^{-1}\left(a_{i}^{\prime}\right)$. Então podemos aplicar a versão análoga (à direita) da proposição (4.13) e concluir que $T$ é um DIDP.

(ii) $\Rightarrow$ (iii) Suponha, por absurdo, que $f T \cap g T=0$. Então $g \notin f T$ e, como $T$ é um DIDP, poderíamos escrever $f T+g T=h T$ para algum $h \in T \backslash f T$, ou seja, $h=f t_{1}+g t_{2}$ para alguns $t_{1}, t_{2} \in T, t_{2} \neq 0$. No entanto, para algum $t \neq 0$ em $T$ teríamos

$$
f=h t=\left(f t_{1}+g t_{2}\right) t=f t_{1} t+g t_{2} t .
$$

Daí $0 \neq g t_{2} t=f\left(1-t_{1} t\right) \in f T \cap g T$, contradição. Logo $f T \cap g T \neq 0$. 
(iii) $\Rightarrow$ (ii) Para todo $d \in D$ existe algum $h \neq 0$ tal que $h \in x T \cap d x T$, então $h=x f=d x g$ para certos $f, g \in T$. Sejam $a x^{n}$ e $b x^{n}$ os termos dominantes de $f$ e $g$, respectivamente. Portanto, o termo dominante de $h$ é $\sigma(a) x^{n+1}=d \sigma(b) x^{n+1}$, daí $\sigma(a)=d \sigma(b)$. Então $d=\sigma\left(a b^{-1}\right) \in \sigma(D)$, logo $\sigma$ é epimorfismo.

Teorema 4.15. Sejam $K$ um corpo, $\sigma \in \operatorname{End}(K)$ e $T=K[x ; \sigma]$. Denote por $F=\{a \in K: \sigma(a)=a\}$ o conjunto dos elementos de $K$ fixados pelo endomorfismo $\sigma$ e por $Z(T)$ o centro do anel $T$.

(i) Se o é um automorfismo de ordem $n$, então temos $Z(T)=F\left[x^{n}\right]$; caso contrário, $Z(T)=F$.

(ii) Todo ideal bilateral de $T$ é da forma $T z x^{m}$ para algum $z \in Z(T) e$ algum inteiro $m \geqslant 0$.

\section{Prova.}

(i) Suponha que $\sigma$ é um automorfismo de ordem $n$; então é claro que $F\left[x^{n}\right] \subseteq Z(T)$. Reciprocamente, suponha que $f=\sum a_{i} x^{i} \in Z(T)$. Então é nulo o comutador $[g, f]=g f-f g$ para todo $g \in T$. Em particular,

$$
0=[x, f]=x f-f x=\sum\left(\sigma\left(a_{i}\right)-a_{i}\right) x^{i+1}
$$

donde $\sigma\left(a_{i}\right)=a_{i}$ para cada $i$ e, portanto, $a_{i} \in F$ para cada $i$. Para todo $a \in K$ temos também

$$
0=[a, f]=a f-f a=\sum a_{i}\left(a-\sigma^{i}(a)\right) x^{i},
$$

$\operatorname{logo} \sigma^{i}(a)=a$ sempre que $a_{i} \neq 0$. Isso implica que $n$ é divisor de $i$ sempre que $a_{i} \neq 0$. Portanto $f \in F\left[x^{n}\right]$ e $F\left[x^{n}\right]=Z(T)$ como queríamos.

Se $\sigma$ não é um automorfismo de ordem $n$, é claro que $F \subseteq Z(T)$. Se $f=\sum a_{i} x^{i} \in Z(T)$ vemos, de forma análoga, que $\sigma^{i}(a)=a$ para todo $a \in K$ sempre que $a_{i} \neq 0$. Como $\sigma$ não é um automorfismo de ordem $n$, vemos que $a_{i} \neq 0$ somente se $i=0 ; \operatorname{logo} f=a_{0} \in F$ e $F=Z(T)$ como queríamos.

(ii) Suponha que $A \subseteq T$ é um ideal e escolha $f=\sum_{i=0}^{t} a_{i} x^{i} \in A \backslash\{0\}$ de grau mínimo t. Pela proposição (4.13) temos $A=T f$ e $f$ tem o mesmo número de termos não-nulos que qualquer outro polinômio em $A$ de grau $t$. Sem perda de generalidade, suponha que $f$ é mônico. Então $a_{t}=1$ e $o$ comutador

$$
[x, f]=x f-f x=\sum_{i=0}^{t}\left(\sigma\left(a_{i}\right)-a_{i}\right) x^{i+1}
$$


tem menos termos que $f$. Logo $[x, f]=0$ pela discussão acima, e portanto $a_{i} \in F$ para cada $i$. Para todo $a \in K$ vemos também que

$$
g=\left(\sigma^{t}(a)\right) f-f a=\sum_{i=0}^{t} a_{i}\left(\sigma^{t}(a)-\sigma^{i}(a)\right) x^{i}
$$

tem grau menor que $t$ e está em $A, \operatorname{logo} g=0$. Daí $a_{i}\left(\sigma^{t}(a)-\sigma^{i}(a)\right)=0$ para cada $i$. Então $\sigma^{t}(a)=\sigma^{i}(a)$ para todo $a \in K$ sempre que $a_{i} \neq 0$, donde $\sigma^{t-i}=1$ sempre que $a_{i} \neq 0$.

Primeiro caso: $\sigma$ é um automorfismo de ordem $n$. Nesse caso, $n$ é divisor de $t-i$ para cada $i$ tal que $a_{i} \neq 0$. Se $m$ denota o grau do termo de menor grau em $f$, então os índices $i$ tais que $a_{i} \neq 0$ são $m, m+n, \ldots, m+q n=t$. Isso quer dizer que $f \in F\left[x^{n}\right] x^{m}=Z(T) x^{m}$, ou seja, $f=z x^{m}$ para algum $z \in Z(T)$.

Segundo caso: $\sigma$ não é um automorfismo de ordem $n$. Nesse caso, $\sigma^{t-i}=1$ só é possível se $i=t, \operatorname{logo} a_{i} \neq 0$ somente se $i=t$. Isso quer dizer que $f=a_{t} x^{t} \in F x^{t}=Z(T) x^{t}$, ou seja, $f=z x^{t}$ para algum $z \in Z(T)$.

O exemplo (4.16) a seguir ilustra os fatos considerados até aqui, ao mesmo tempo em que faz uso de idéias que serão úteis no exemplo (4.17), que é nosso objetivo principal no momento.

Exemplo 4.16. Um anel de polinômios skew $K[x ; \sigma]$ onde $K$ é um corpo e $\sigma$ é um endomorfismo não-sobrejetor de $K$.

Seja $K=\mathbb{Q}(x)$ o corpo de funções racionais em $x$ sobre $\mathbb{Q}$ e seja $\sigma$ em End $(K)$ definido por $\sigma(x)=x^{2}$. Claramente $\sigma$ não é um epimorfismo. Então o anel de polinômios $T=K[\lambda ; \sigma]$ na variável $\lambda$ é um DIEP pela proposição (4.13), mas não é um DIDP pela proposição (4.14). Pelo teorema (4.15) os ideais próprios de $T$ são da forma $\left\langle\lambda^{m}\right\rangle$ para algum inteiro positivo $m$. Assim, os ideais de $T$ formam um cadeia totalmente ordenada.

Exemplo 4.17. Um anel que tem um número finito de órbitas sob a ação regular à esquerda, mas infinitas órbitas sob a ação regular à direita.

Seja $K$ um corpo de característica diferente de 2 e $K(x)$ o corpo de funções racionais em $x$ sobre $K$, e seja $\sigma \in \operatorname{End}(K)$ definido por $\sigma(x)=x^{2}$. Considere o anel $S=K(x)[Y ; \sigma]$ e construa o quociente $R=S /\left\langle Y^{2}\right\rangle$. Então $R=K(x)+K(x) y$, onde $y$ denota a classe $Y+\left\langle Y^{2}\right\rangle$.

Tome $f(x) \in K(x)$. Definindo

$$
f_{1}(x)=\frac{f(x)+f(-x)}{2} \quad \text { e } \quad f_{2}(x)=\frac{f(x)-f(-x)}{2}
$$


é fácil ver que $f(x)=f_{1}(x)+f_{2}(x)$, que a expressão de $f_{1}(x)$ só tem potências pares de $x$ e que a expressão de $f_{2}(x)$ só tem potências ímpares de $x$ no numerador e potências pares de $x$ no denominador. Portanto, temos

$$
R=K(x)+K(x) y=K(x)+y K(x)+x y K(x) .
$$

Pela proposição (4.13), todo ideal à esquerda de $R$ é da forma $R \varphi$ para algum $\varphi \in R$. Se $\varphi=f(x)+g(x) y \operatorname{com} f(x) \neq 0$ então

$$
[f(x)+g(x) y]\left[\frac{1}{f(x)}-\frac{g(x)}{f(x) \sigma(f(x))} y\right]=1,
$$

$\operatorname{logo} J(R)=R y$ é o único ideal à esquerda de $R$ além de 0 e $R$. Concluímos, portanto, que $R$ é uniserial à esquerda. Pelo teorema (4.7), $R$ tem um número finito de órbitas sob a ação regular à esquerda.

Pelo teorema (4.15), todo ideal bilateral de $S$ é da forma $\left\langle Y^{m}\right\rangle$. Logo, pelo teorema da correspondência, o único ideal bilateral de $R$ é $R y$. Portanto, $R$ é um anel indecomponível. Suponha, por absurdo, que $R$ tenha um número finito de órbitas sob a ação regular à direita. Pela versão "à direita" do teorema (4.7), $R$ seria uma soma direta de anéis uniseriais à direita mais um anel finito. Como $R$ é indecomponível e não é finito, então $R$ deveria ser uniserial à direita. Mas isso não é possível, pois os ideais à direita $A=y R \mathrm{e}$ $B=x y R$ não são comparáveis por inclusão. De fato, é claro que $x y \notin A \mathrm{e}$ que $y \notin B, \log A \not \subset B$ e $B \not \subset A$. Logo, $R$ tem um número infinito de órbitas sob a ação regular à direita. 


\section{Referências Bibliográficas}

[AC] H. Achkar, Sur les Anneaux Aritmétiques à Gauche.

Acad. Sci. 278, n 5 (1974) 307-309.

[BA] H. Bass, Finitistic Dimension and a Homological Generalization of Semi-primary Rings.

Trans. Amer. Math. Soc. 95 (1960) 466-488.

[BE] E.A. Behrens, Ring Theory.

Academic Press, Inc., New York, 1972.

[BE I] E.A. Behrens, Distributiv Darstellbare Ringe I.

Math. Z. 73, n 5 (1960) 409-432.

[BE II] E.A. Behrens, Distributiv Darstellbare Ringe II. Math. Z. 76, no 4 (1961) 367-384.

[BR] H. H. Brungs, Rings with Distributive Lattice of Right Ideals.

J. Algebra $40 \mathrm{n}^{\circ} 2$ (1976) 392-400.

[CA] V. Camillo, Distributive Modules. Journal of Algebra 36 (1975) 16-25.

[CO] P.M. Cohn, Universal Algebra.

Harper and Row, New York, 1965.

[DF] D.S. Dummit e R.M. Foote, Abstract Algebra (second edition). John Wiley \& Sons, New York, 1999.

[FD] B. Farb e R. Keith Dennis, Noncommutative Algebra. Springer-Verlag, New York, 1993.

[HA] J. Han, Regular Action in a Ring with a Finite Number of Orbits. Comm. Algebra 25 (1997) 2227-2236. 
[HI] Y. Hirano, Rings with finitely many orbits under the regular action. Rings, Modules, Algebras and Abelian Groups, a Dekker Series of Lecture Notes in Pure and Applied Mathematics 236 (2004) 343-347. M. Dekker, New York.

[HU] T. Hungerford, Algebra.

Springer-Verlag, New York, 1974.

[JO] D. Jonah, Rings with the Minimum Condition for Principal Right Ideals Have the Maximum Condition for Principal Left Ideals.

Math. Z. 113 (1970) 106-112.

[LA] T. Y. Lam, A First Course in Noncommutative Rings. Springer-Verlag, New York, 1991.

[LP] R. Lidl e G. Pilz, Applied Abstract Algebra. Springer-Verlag, New York, 1998.

[PS] C. Polcino Milies e S.K. Sehgal, An Introduction to Group Rings. Kluwer Academic Publishers, Dordrecht, 2002.

[RO I] L.H. Rowen, Ring Theory, volume I. Academic Press, Inc., San Diego, 1988.

[RO II] L.H. Rowen, Ring Theory, volume II. Academic Press, Inc., San Diego, 1988.

[ST I] W. Stephenson, Modules whose Lattice of Submodules is Distributive. Proc. London Math. Soc. 28 (1974) 291-310.

[ST II] W. Stephenson, Lattice Isomorphisms between Modules (l) Endomorphism Rings.

J. London Math. Soc. (2) 1 (1969) 177-183.

[TU] A. Tuganbaev, Distributive Modules and Related Topics. Gordon and Breach Science Publishers, Amsterdam, 1999.

[VE] E. M. Vechtomov, Rings of Continuous Functions and F-Spaces, Selected Themes.

Moscow State Teachers' Training University Press, Moscow, 1992.

[ZE] D. Zelinsky, Raising Idempotents.

Duke Math. J. 21 (1954) 315-312. 
[ZH] V. V. Zharinov, Distributive Lattices and their Application in Complex Analysis.

Trudy Mat. Inst. Akad. Nauk SSSR, Mockba 162 (1983) 3-116. 